Sebastian Schäfer

FABRY-PÉROT ETALONS FOR HIGH PRECISION RADIAL VELOCITY CALIBRATION

DisSERTATION

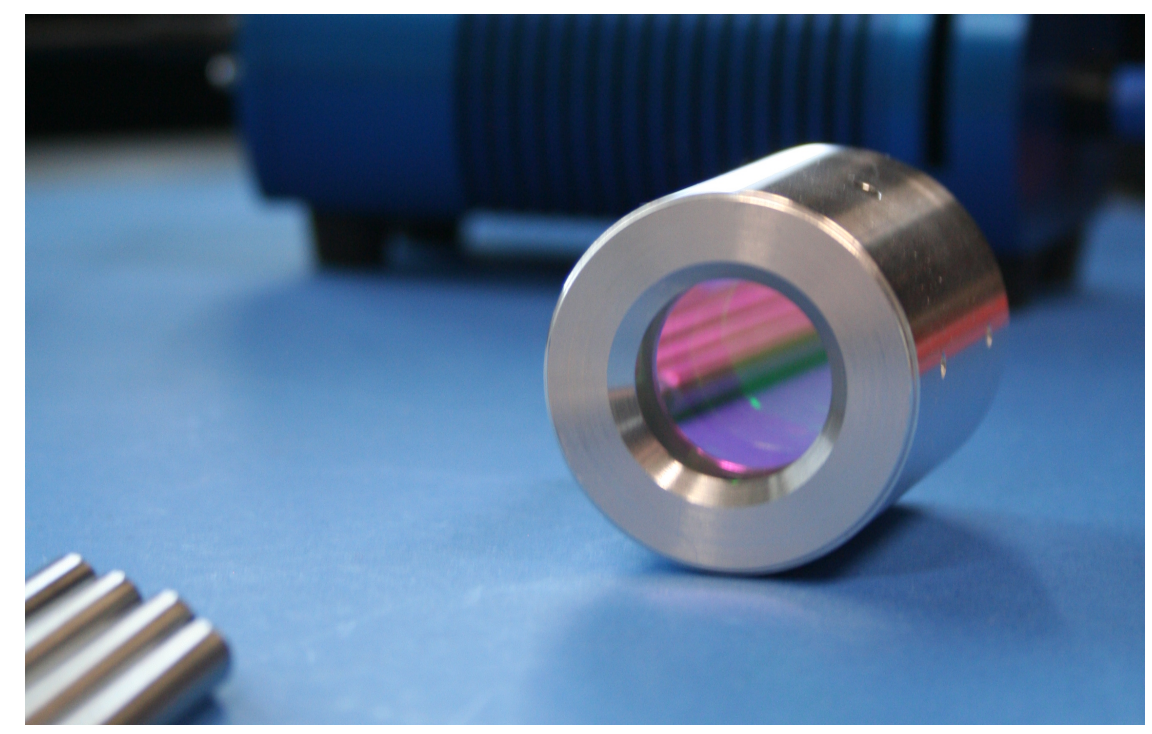





\title{
Fabry-Pérot etalons for high precision radial velocity calibration
}

\author{
Dissertation \\ zur Erlangung des mathematisch-naturwissenschaftlichen Doktorgrades \\ "Doctor rerum naturalium" \\ der Georg-August-Universität Göttingen \\ im Promotionsprogramm GAUSS \\ der Georg-August University School of Science (GAUSS)
}

vorgelegt von

Sebastian Schäfer

aus

Lübeck

Göttingen, 2014 


\section{Betreuungsausschuss}

Prof. Dr. Ansgar Reiners, Sonnenphysik und Stellare Astrophysik, Institut für Astrophysik

Prof. Dr. Stefan Dreizler, Sonnenphysik und Stellare Astrophysik, Institut für Astrophysik Mitglieder der Prüfungskommission

Referent/in: Prof. Dr. Ansgar Reiners, Sonnenphysik und Stellare Astrophysik, Institut für Astrophysik

Korreferent/in: Prof. Dr. Stefan Dreizler, Sonnenphysik und Stellare Astrophysik, Institut für Astrophysik

Weitere Mitglieder der Prüfungskommission:

Prof. Dr. Wolfgang Glatzel, Sonnenphysik und Stellare Astrophysik, Institut für Astrophysik

Prof. Dr. Wolfram Kollatschny, Extragalaktische Astrophysik und Kosmologie, Institut für Astrophysik

Prof. Dr. Jens Niemeyer, Extragalaktische Astrophysik und Kosmologie, Institut für Astrophysik

Prof. Dr. Claus Ropers, Nano-Optik und ultraschnelle Dynamik, IV.Physikalisches Institut

Tag der mündlichen Prüfung: 


\section{Abstract}

The current generation of astronomical spectrographs can measure radial velocity signals of exoplanets down to a few $\mathrm{ms}^{-1}$, in ideal cases even $60 \mathrm{~cm} \mathrm{~s}^{-1}$ can be achieved. This led to the discovery of rocky planets with masses of only a few times the mass of the Earth, orbiting their host stars within days or weeks. However, Earth only produces a radial velocity shift of about $9 \mathrm{~cm} \mathrm{~s}^{-1}$ during its one year orbit around the Sun. Thus, the radial velocity precision of astronomical spectrographs has to be improved by at least one magnitude in order to find these planets, even though the radial velocity signal of Earth-like planets in the habitable zone of low mass stars would be larger.

Currently, a large fraction of the radial velocity uncertainty of astronomical spectrographs is due to the wavelength calibration. The established methods, e.g. using Hollow Cathode emission lamps as a wavelength reference, do not provide the precision needed for finding a second Earth. They also do not work particularly well in certain wavelength regions which could otherwise be used to find planets, for example the near infrared.

One of the candidates for an improved wavelength calibration at the sub $\mathrm{m} \mathrm{s}^{-1}$ level, especially in the near infrared, are Fabry-Pérot etalons. This thesis explains how a Fabry-Pérot unit was developed for the upcoming CARMENES spectrograph and how it will be able to achieve a radial velocity stability of $10 \mathrm{~cm} \mathrm{~s}^{-1}$. Simulations of the impact of temperature, pressure and mechanical stability on the resulting radial velocity performance have been made. The results confirm the feasibility of using a Fabry-Pérot unit for the wavelength calibration of an astronomical spectrograph with a radial velocity precision of the order of $10 \mathrm{~cm} \mathrm{~s}^{-1}$ and provide first requirements for the unit.

Additionally, the effect of fibers in combination with Fabry-Pérots has been investigated. A close relation between certain fiber properties and the resulting spectrum has been found which impacts the radial velocity precision of Fabry-Pérot units in general. The final setup of the Fabry-Pérot unit for CARMENES was designed and developed accordingly. The setup and the components used are explained and first light measurements are shown. 



\section{Contents}

1 Introduction 1

2 Wavelength calibration $\quad \mathbf{7}$

2.1 Hollow Cathode Lamps . . . . . . . . . . . . . . . . . . . . 7

2.2 Laser Frequency Comb . . . . . . . . . . . . . . . . . . . . . . . . 8

2.3 Fabry-Pérot etalon . . . . . . . . . . . . . . . . . . . 10

3 CARMENES 11

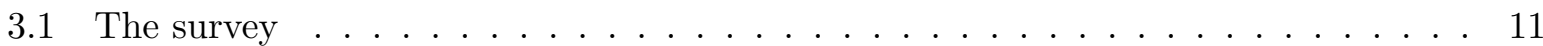

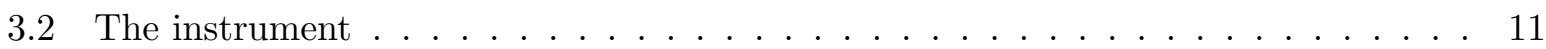

3.3 Wavelength calibration with CARMENES . . . . . . . . . . . . . 12

4 Fabry-Pérot Etalon: Simulations \& test setup 15

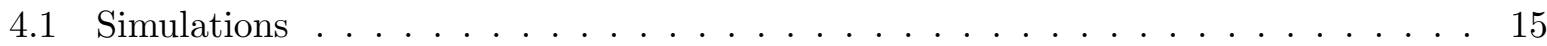

4.1 .1 Stability of the mirror distance $l \ldots \ldots \ldots \ldots \ldots \ldots$

4.1 .2 Stability of the angle $\theta \ldots \ldots \ldots \ldots \ldots \ldots$

4.1 .3 Stability of the index of refraction $n \ldots \ldots \ldots \ldots$

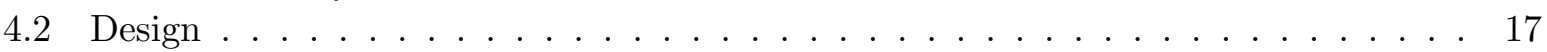

4.2.1 Optimization for

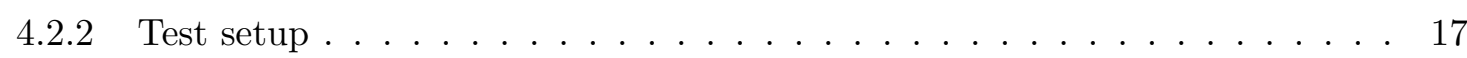

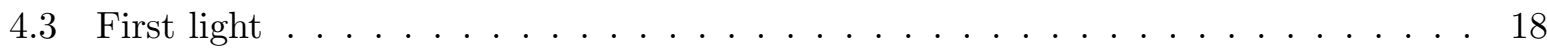

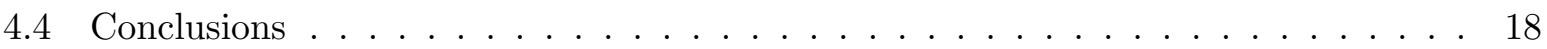

5 Fabry-Pérot Etalon: Fibers and their illumination 21

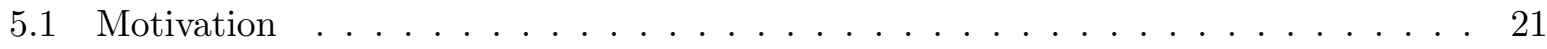

5.2 A real Fabry-Pérot . . . . . . . . . . . . . . . . . . . . . . 21

5.3 Laboratory measurements . . . . . . . . . . . . . . . . . . . . . . . . 24

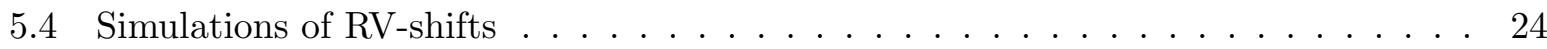

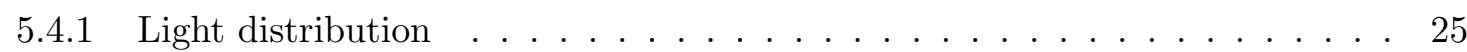

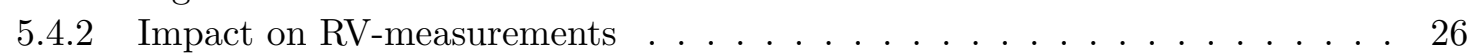

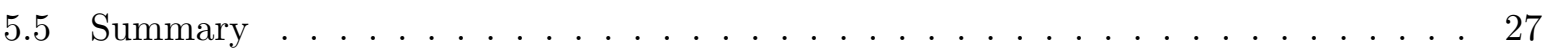

6 Fabry-Pérot Etalon: Final setup for CARMENES 29

6.1 Vacuum tank . . . . . . . . . . . . . . . . . . . . . . 29

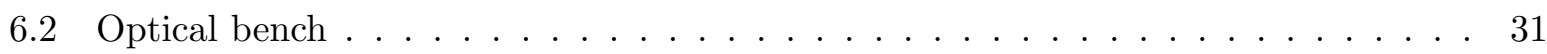

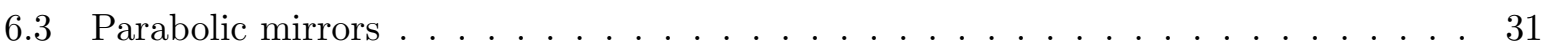

vii 


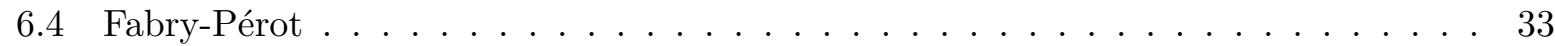

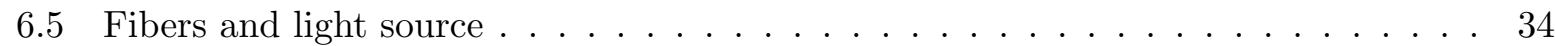

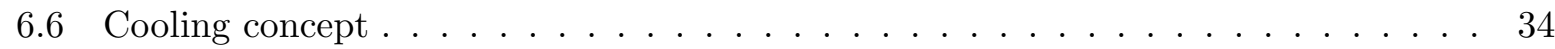

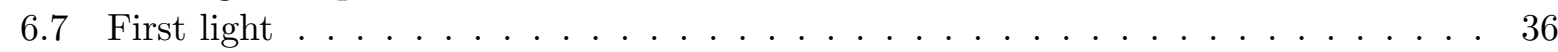

$\begin{array}{llr}7 & \text { XSHOOTER } & 39\end{array}$

7.1 Introduction \& connection to CARMENES . . . . . . . . . . . . . . . 39

7.2 Observations and data reduction . . . . . . . . . . . . . . . . . . . 41

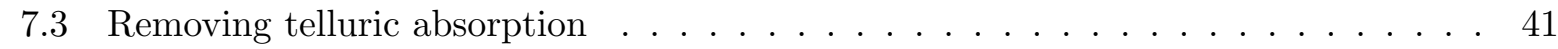

7.4 Spectral line identification . . . . . . . . . . . . . . . . . . . 41

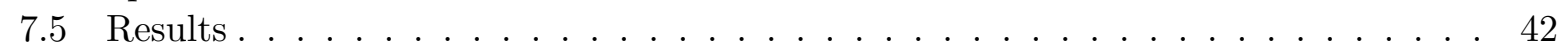

8 Conclusion \& Outlook $\quad 65$

$\begin{array}{ll}\text { Bibliography } & 67\end{array}$ 


\section{List of Figures}

1.1 Functional principle of the RV method. The host star and its planet orbit their common center of gravity. Thereby the light of the star is being red- or blue shifted by the Doppler effect, depending on its motion (source: ESO). . . . . . . . . . 2

1.2 Habitable zone for different stellar masses, e.g. for Gliese 581 and the Sun (source:

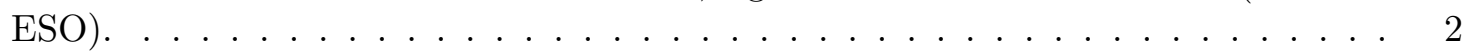

1.3 HARPS radial velocity measurements of HD 69830, showing three planets (source:

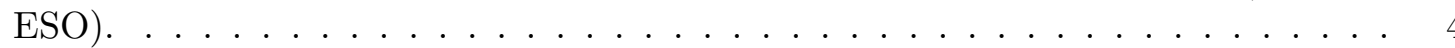

1.4 Measurements of the visual FP with the historic spectrograph with the improvements of the signal to noise ration of the FP due to increased coupling efficiency (from left to right). The red line is the mean spectrum of ten single exposures, shown in black. . . . . . . . . . . . . . . . . . . . . .

2.1 Schematic design of a hollow cathode lamp. Source: Klose and Bridges (1987) . . . 9

2.2 Small section of a CCD image of the spectrum of both a ThAr emission lamp and a Fabry-Pérot etalon with an artificial offset of 10 pixels. . . . . . . . . . . 9

2.3 Sketch of the LFC setup tested at HARPS. Source: Nature (Wilken et al., 2012). . 9

3.1 Layout of the VIS channel optical design (source: CARMENES optics Final Design Review document FDR-02A, VIS channel optics design) . . . . . . . . . . . . . . 12

4.1 Transmission function of a theoretical $\mathrm{FP}$ with $F=8, l=9.99 \mathrm{~mm}, n=1$ and

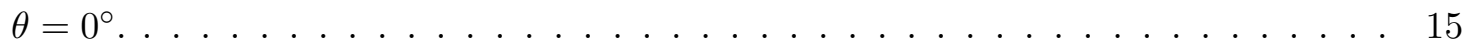

4.2 The same FP as in Fig. 4.1 but with the resolution and pixel sampling of CARMENES

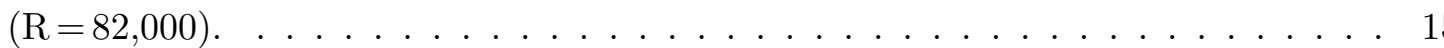

4.3 RV-precision in $\mathrm{m} \mathrm{s}^{-1}$ as function of temperature stability with respect to thermal

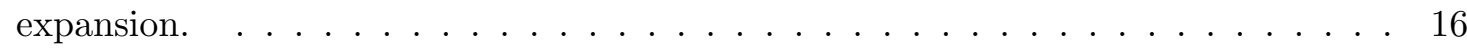

4.4 RV-precision in $\mathrm{m} \mathrm{s}^{-1}$ as function of angle stability. . . . . . . . . . 16

4.5 Left: RV-deviation in $\mathrm{m} \mathrm{s}^{-1}$ at $\lambda=900 \mathrm{~nm}, \mathrm{p}=1$ bar and $\mathrm{T}=20^{\circ} \mathrm{C}$ due to changes in pressure and temperature. Note the temperature scale is the same as for the thermal expansion (Figure 4.3). Right: Same plot but with $\mathrm{p}=1$ mbar. . . . . . . . 17

4.6 The intrinsic Doppler error as a function of the FP's spacing. The minimum and therefore the optimal value is different for the two arms of CARMENES. Solid line: optical arm, dashed line: NIR arm. . . . . . . . . . . . . . . 18

4.7 3D image of the FP-unit. The FP itself is located inside the lens tube, which is mounted in a cage construction. . . . . . . . . . . . . . . . . . 19

4.8 Sketch of the FP-unit. . . . . . . . . . . . . . . . . . . . . . . . . 19 
4.9 Raw frames of the Tautenburg echelle spectrograph. Parts of the spectra of both the ThAr-lamp (top) and the Fabry-Perot (bottom) are shown to illustrate the difference in line density and brightness homogeneity. . . . . . . . . . . . .

5.1 Schematic overview of the test setup. . . . . . . . . . . . . .

5.2 Left: Perimeter of the illuminated area for different fiber diameters. Right: Simulation of transmission spectra of a Fabry-Pérot for different fiber diameters assuming a Gaussian light distribution on the fiber. . . . . . . . . . . . . . .

5.3 FTS measurements of a Fabry-Pérot using different fiber diameters $(R=1,000,000$

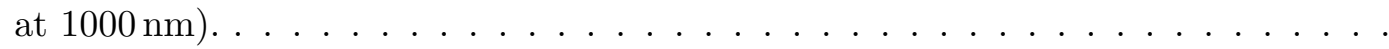

5.4 Contour of the circular light distribution of a $400 \mu \mathrm{m}$ fiber with offsets of 4,8 and $20 \mu \mathrm{m}(1,2$ and $5 \%$ of the fiber diameter $) \ldots \ldots \ldots \ldots \ldots$

5.5 Illuminated area as a function of distance $D$ from the fiber midpoint (see Eq. 5.1) for different offsets of the light distribution on the exit of a $400 \mu \mathrm{m}$ fiber (see Fig. 5.4). This weighting function is then used to add up the different spectra for each annulus in order to get the total spectrum (Fig. 5.6) . . . . . . . . . . .

5.6 Simulated FP transmission spectra for a $400 \mu \mathrm{m}$ fiber and an offset of 1,2 and $5 \%$ at the fiber exit and the resulting RV-error. The barycenter for each offset is marked by a vertical line. . . . . . . . . . . . . . . . . . 26

5.7 Resulting RV-errors due to an offset of the light distribution on the fiber exit. . . . 27

6.1 Left: CAD drawing of the tank. Right: Tank connected with vacuum pump (silver hose) and thermopump (red hoses). . . . . . . . . . . . . . . . .

6.2 Left: CAD drawing of the DN $40 \mathrm{CF}$ fiber feedthrough flange. Right: The assembled vacuum feedthrough with fiber protection tubes on both sides. . . . . . . .

6.3 CAD-drawing of the optical bench. The off-axis parabolic mirrors are directly screwed into blocks of Aluminum (shown in orange), the FP (green) is mounted inside the middle block (blue) and held in place by one screw. . . . . . . . . . .

6.4 The optical bench standing with both its off-axis parabolic mirrors attached and the FP fixed inside the middle block. . . . . . . . . . . . . . . . . 33

6.5 Photo of the blue Fabry-Pérot. . . . . . . . . . . . . . . . . 33

6.6 The LDLS Principle of operation. . . . . . . . . . . . . . . . . . 35

6.7 Left: Section view of a CAD drawing of the vacuum tank. The $46 \mathrm{~mm}$ wide gap between the outer (blue) and inner layer (red) contains the silicon oil which circulates upwards (inlet and outlet hose attachments not shown). Right: The Huber Ministat 230 cooling circulator. . . . . . . . . . . . . . . . .

6.8 Part of the CCD raw image of the visual FP, using a Halogen lamp and a $50 \mu \mathrm{m}$ fiber (taken at Tautenburg with $\mathrm{R}=67,000$ and the IR setup of the spectrograph).

6.9 First light of the calibration unit in combination with the FP unit. The 'wheel' containing the calibration lamps is in the center, the calibration unit with its electronics is on the left and in the right background the Halogen lamp of the FP unit and one of the fibers can be seen. . . . . . . . . . . . . . . .

7.1 Schematic overview of XSHOOTER. Each of the arms is an independent cross dispersed echelle spectrograph with it's own slit and, with the exception of the NIR arm, its own ADC. Picture taken from ESO. . . . . . . . . . . . . . . . . . 40

7.2 Wavelength range: $350-450 \mathrm{~nm}$. Threshold for the line identification: $0.997 \ldots$. . . 43

7.3 Wavelength range: $450-550 \mathrm{~nm}$. Threshold for the line identification: $0.975 \ldots \ldots 4$ 
7.4 Wavelength range: $550-650 \mathrm{~nm}$. Threshold for the line identification: $0.9 \ldots \ldots 45$

7.5 Wavelength range: $650-750 \mathrm{~nm}$. Threshold for the line identification: 0.7 . . . . 46

7.6 Wavelength range: $750-850 \mathrm{~nm}$. Threshold for the line identification: 0.6 . . . . 47

7.7 Wavelength range: $850-950 \mathrm{~nm}$. Threshold for the line identification: 0.5 . . . . . 48

7.8 Wavelength range: $950-1050 \mathrm{~nm}$. Threshold for the line identification: 0.3. Note the overlap between the VIS and NIR channel between $995 \mathrm{~nm}$ and $1015 \mathrm{~nm}$. . . . 49

7.9 Wavelength range: $1050-1150 \mathrm{~nm}$. Threshold for the line identification: $0.3 \ldots$. . . 50

7.10 Wavelength range: $1150-1250 \mathrm{~nm}$. Threshold for the line identification: 0.5 . . . . 51

7.11 Wavelength range: $1250-1350 \mathrm{~nm}$. Threshold for the line identification: $0.5 \ldots$. . . 52

7.12 Wavelength range: $1350-1450 \mathrm{~nm}$. Threshold for the line identification: 0.99 (no lines identified). . . . . . . . . . . . . . . . 53

7.13 Wavelength range: $1450-1550 \mathrm{~nm}$. Threshold for the line identification: $0.4 \ldots \ldots$. . . 54

7.14 Wavelength range: $1550-1650 \mathrm{~nm}$. Threshold for the line identification: 0.3 . . . . 55

7.15 Wavelength range: $1650-1750 \mathrm{~nm}$. Threshold for the line identification: $0.2 \ldots$. . . 56

7.16 Wavelength range: $1750-1850 \mathrm{~nm}$. Threshold for the line identification: 0.99 (no lines identified). . . . . . . . . . . . . . . . . . 57

7.17 Wavelength range: $1850-1950 \mathrm{~nm}$. Threshold for the line identification: 0.6 . . . . 58

7.18 Wavelength range: $1950-2050 \mathrm{~nm}$. Threshold for the line identification: 0.7 . . . . 59

7.19 Wavelength range: $2050-2150 \mathrm{~nm}$. Threshold for the line identification: 0.25 . . . 60

7.20 Wavelength range: $2150-2250 \mathrm{~nm}$. Threshold for the line identification: $0.4 \ldots \ldots 1$

7.21 Wavelength range: $2250-2350 \mathrm{~nm}$. Threshold for the line identification: 0.45 . . . 62

7.22 Wavelength range: $2350-2450 \mathrm{~nm}$. Threshold for the line identification: 0.5 . . . . 63 



\section{Chapter 1}

\section{Introduction}

Finding planets around other stars than our sun has been a driver for both technology and scientific methods. Since the discovery of the first exoplanet around a Sun-like star by Mayor and Queloz (1995) more than 1000 planets in over 800 planetary systems have been found ${ }^{1}$ with a variety of methods and telescopes. Detected planetary masses range from over $20 \mathrm{M}_{\text {Earth }}$ (e.g. Sato et al., 2010), which technically falls into the brown dwarf mass regime, down to below $2 \mathrm{M}_{\text {Earth }}$ (Mayor et al., 2009; Dumusque et al., 2012). However, all masses for detections with the radial velocity method are lower limits due to the unknown inclination angle.

There are different ways to find exoplanets, e.g. direct imaging of the planet, microlensing, transit observations or the radial velocity (RV) method. The RV method makes use of the Doppler effect, which changes the frequency of the light of moving objects (blue shifted for approaching objects and red shifted for receding objects), see Fig. 1.1 for an illustration. The relationship between the observed frequency $f$ and the emitted frequency $f_{0}$ for velocities much lower than the speed of light is given by:

$$
f=\left(1+\frac{v_{r}-v_{s}}{c}\right) f_{0}
$$

where $c$ is the speed of light and $v_{r}-v_{s}$ is the velocity of the receiver relative to the source (negative if they are moving away from

\footnotetext{
1 www.exoplanet.eu
}

each other). The host star and its planet are orbiting around their common center of gravity, which is usually inside the diameter of the star itself due to its large mass compared to the planet. Hence, if the movement of that star has a component towards (or away from) the earth, the light of that star is blue- or red shifted. If one observes the light from such a planet hosting star using a spectrograph, one can see the spectral lines of the star being shifted back and forth compared to a calibration source that is moving with the observer (e.g. earths atmospheric lines or hollow cathode lamps like ThAr). Measuring the shift in frequency therefore gives the relative speed between the observer and the star by using Eq. 1.1. Kepler's third law can be used to determine the distance of the planet to the star and Newton's law of gravitation provides the velocity of the planet and finally the planetary mass as a lower lower limit.

The amplitude of the RV signal scales with both inverse distance and mass of the planet:

$$
K_{\mathrm{p}}=0.0895 \mathrm{a}^{-\frac{1}{2}} M_{\mathrm{s}}^{-\frac{1}{2}} M_{\mathrm{p}} \sin (i)
$$

with $K_{\mathrm{p}}$ being the RV amplitude in $\mathrm{m} \mathrm{s}^{-1}$, the semi-major axis a in AU, the stellar mass in solar masses $M_{\mathrm{s}}$, the planetary mass in Earth masses $M_{\mathrm{p}}$ and the inclination angle $i$. The inclination angle can not be determined by the RV method and therefore all resulting planetary masses for exoplanets are lower limits.

For example the RV signal of the Sun due to the presence of Jupiter is $12.7 \mathrm{~m} \mathrm{~s}^{-1}$ over a 


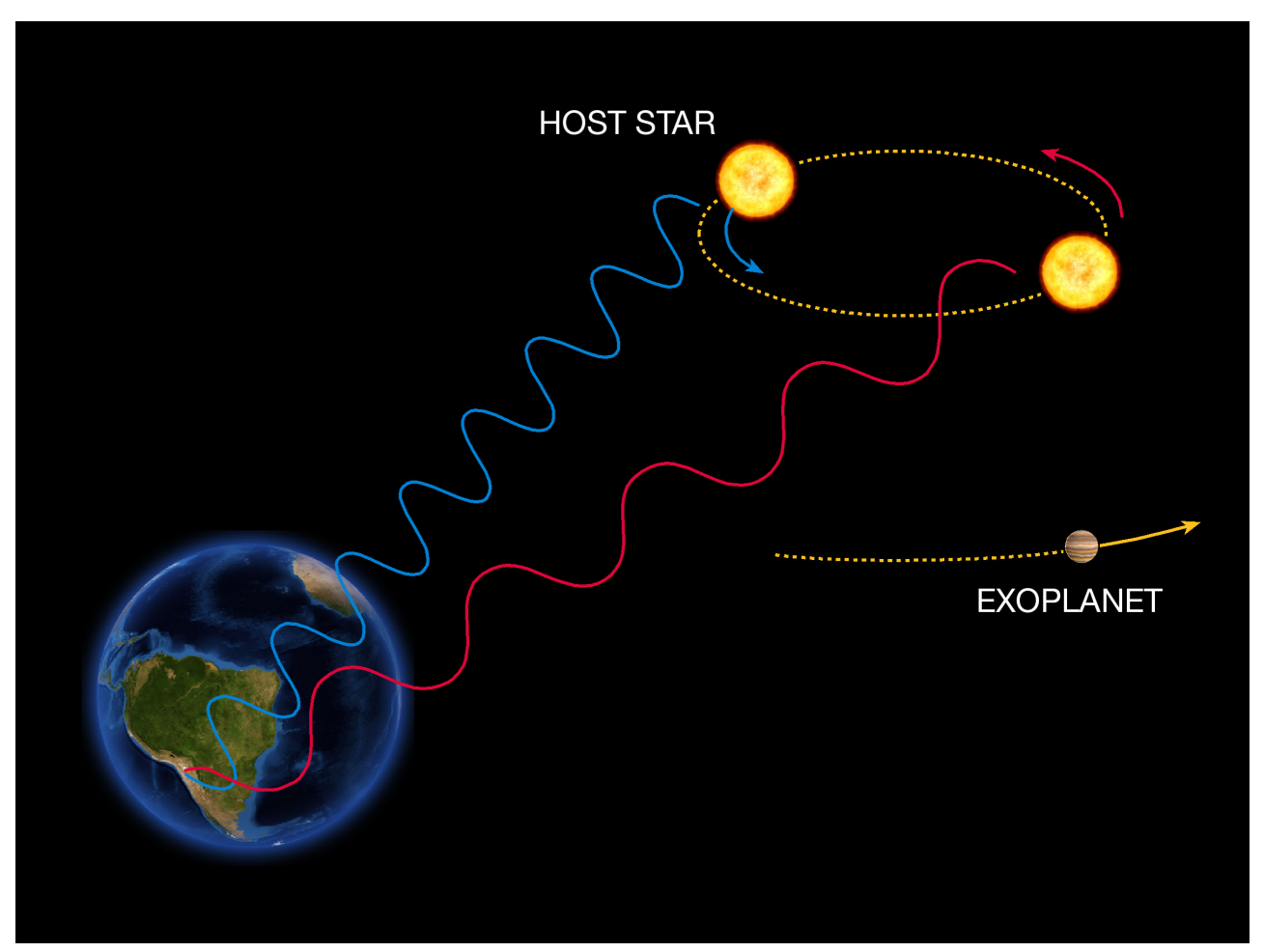

Figure 1.1: Functional principle of the RV method. The host star and its planet orbit their common center of gravity. Thereby the light of the star is being red- or blue shifted by the Doppler effect, depending on its motion (source: ESO).

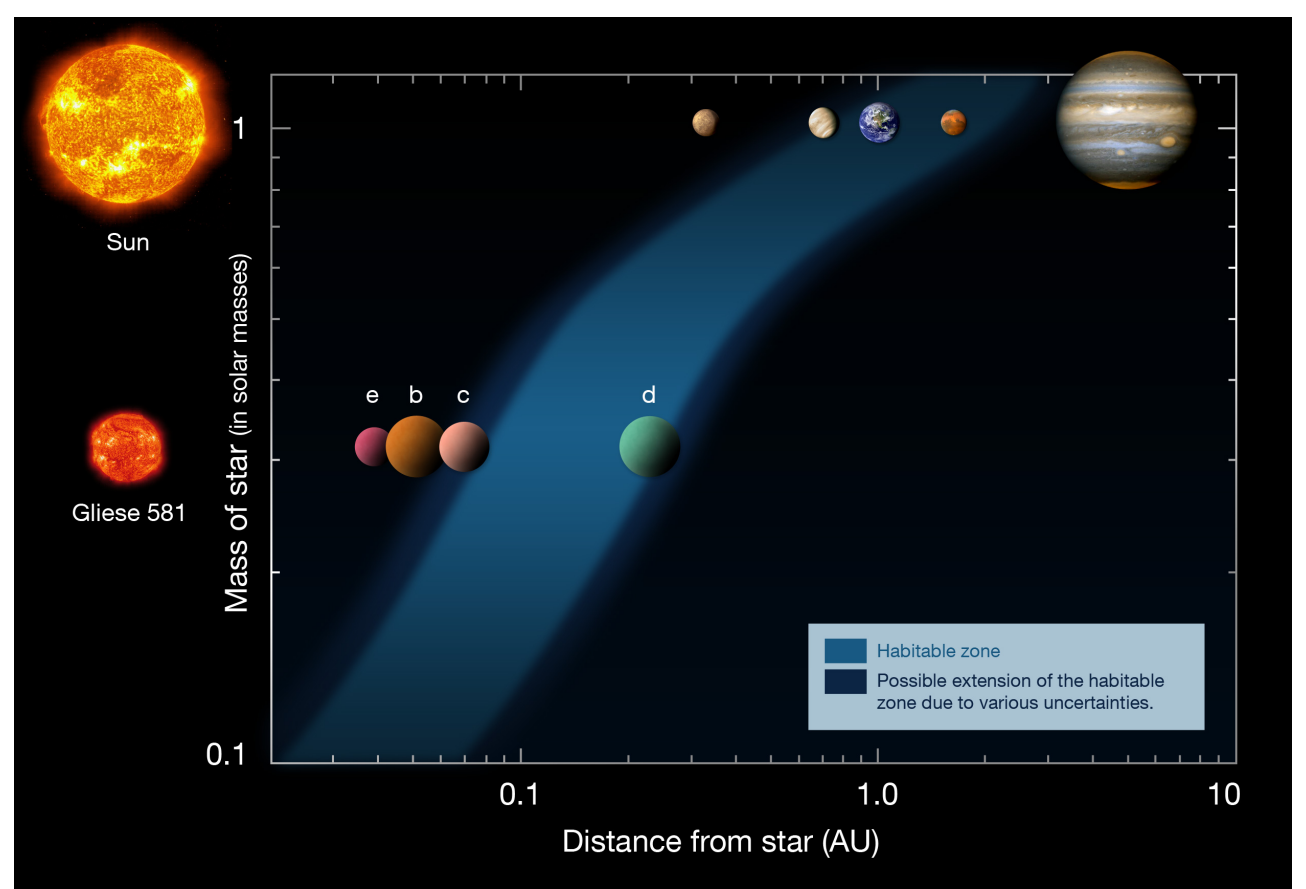

Figure 1.2: Habitable zone for different stellar masses, e.g. for Gliese 581 and the Sun (source: ESO). 
period of 12 years while our Earth only produces a velocity of $0.09 \mathrm{~m} \mathrm{~s}^{-1}$ over one year. Putting Jupiter at a $1 \mathrm{AU}$ orbit would increase the RV shift of the Sun to $28.4 \mathrm{~m} \mathrm{~s}^{-1}$ and a so called Super-Earth with a mass of $5 \mathrm{M}_{\text {Earth }}$ at an orbit of $0.1 \mathrm{AU}$ would result in a $1.4 \mathrm{~m} \mathrm{~s}^{-1}$ RV signal.

Particularly interesting are planets inside the habitable zone (HZ) of a star. The HZ is defined as the region around a star within which a planet with sufficient atmospheric pressure can support liquid water on its surface. For example Mars is well within the HZ of our Sun, just like the Earth, but does not have the atmospheric pressure to create a greenhouse effect and therefore sustain water on its surface. As shown in Fig. 1.2 the HZ is closer to the star for low mass stars, e.g. for M-type stars. Thus it is much easier to find a rocky planet (between 1 and $5 \mathrm{M}_{\text {Earth }}$ ) in the $\mathrm{HZ}$ of a low mass star than around a sun-like star since the RV amplitude is much higher for close in planets (see Eq. 1.2). Additionally, their orbital period is much shorter - while the Earth is orbiting the Sun in one year these planets are orbiting their host star within days or a few weeks. Therefore finding their RV signal does not necessarily require permanent observations over years but only weeks.

In order to use the RV method one has to measure the spectrum of the target star with a spectrograph. Astronomical spectrographs exist in many different designs, however the basic principle is almost always the same: The light from the telescope is sent to an echelle grating as the dispersive element and a detector (e.g. a CCD for visual or a CMOS for infrared light) is then used to measure the light intensity as a function of wavelength. In many cases the light is also send through a cross disperser in order to distribute the echelle orders over the detector to get a more efficient wavelength coverage per exposure on the detector chip.

The realization of the basic concept is different for every spectrograph. Different optical elements are used depending on the de- sired features of the spectrograph in question (see e.g. Figs. $3.1 \& 7.1$ for the XSHOOTER and CARMENES designs). Some spectrographs are directly mounted at the telescope (for example XSHOOTER (Vernet et al., 2011)), some are fiber fed (e.g. HARPS (Mayor et al., 2003)) and some do also imaging and polarimetry (e.g. FORS2 (Appenzeller et al., 1998)). For high precision RV measurements the following requirements should be fulfilled: High resolution (at least $R=\frac{\Delta \lambda}{\lambda}=50,000$ ), sufficient pressure and temperature stabilization, mechanical stability and a wavelength calibration which is able to correct for instrumental drifts. High resolution is needed to measure the spectral stellar lines with a precision high enough to actually see the blue- or redshift. High stability is required so that the RV signal is not lost in the noise of the drift of the spectrograph itself due to changes of external parameters like temperature and pressure. Finally, the wavelength calibration has to be better than the RV amplitude that is going to be measured.

The best stability is usually achieved by using a fiber fed spectrograph inside a temperature stabilized vacuum tank. The prime example for this design is HARPS (Mayor et al., 2003) at the $3.6 \mathrm{~m}$ telescope at La Silla. It is an instrument dedicated to the discovery of extrasolar planets with the RV method with a long term RV accuracy on the order of 1 $\mathrm{m} \mathrm{s}^{-1}$ and better. HARPS is using the simultaneous Thorium reference method (Baranne et al., 1996), meaning one of its two fibers is fed with light from the telescope while the second one is fed with light from a calibration source (usually a ThAr lamp). Both spectra end up next to each other on the CCD and the calibration spectrum can be used to create a wavelength solution and also to correct for instrumental drifts in between measurements. This has proven to be an ideal setup for RV measurements with RV precisions down to $20 \mathrm{~cm} \mathrm{~s}^{-1}$ for one night (Lovis and Pepe, 2007). Figure 1.3 shows HARPS RV measurements of HD 69830 with the phase 


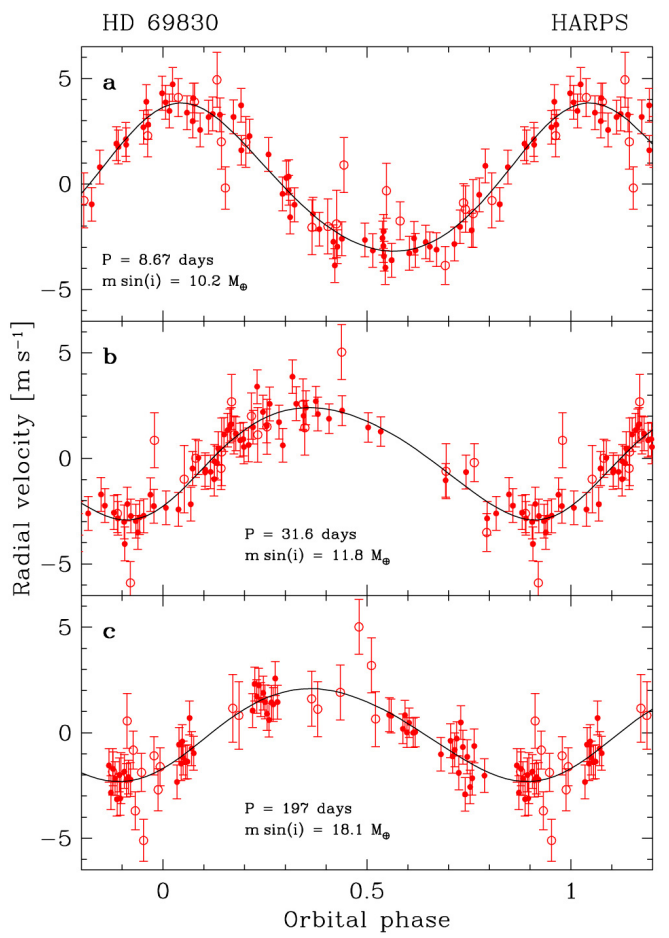

Figure 1.3: HARPS radial velocity measurements of HD 69830, showing three planets (source: ESO).

folded RV signals of three planets planet with periods of $8.67,31.6$ and 197 days, respectively. The corresponding minimal masses are 10.2, 11.8 and 18.1 Earth masses.

However, HARPS is only covering the spectral range between $378 \mathrm{~nm}-691 \mathrm{~nm}$ while Mtype stars, which may provide a better target to find Earth-size planets inside the HZ, emit most of their light in the near-infrared (NIR). Observing in the NIR (in the context of this work roughly $800 \mathrm{~nm}-1800 \mathrm{~nm}$ ) also means other calibration sources are required because ThAr does not provide enough calibration lines in that wavelength region to reach the same level of RV precision as in the visual. One of the candidates for an improved wavelength calibration at the sub- $\mathrm{m} \mathrm{s}^{-1}$ level, particularly in the NIR, are Fabry-Pérot etalons (FP). FPs are typically made of two parallel, highly reflective mirrors. Their transmission spectrum show peaks of high intensity corre- sponding to the distance of the mirrors, so in principle, the line density of a FP can be chosen freely. Thus FPs can provide the ideal number of peaks (with respect to the resolution element size of the spectrograph) over a broad wavelength range, while the bandwidth is only limited by the coating of the mirrors and the light source used. Therefore a number of upcoming spectrographs meant for high precision RV measurements are considering or already decided to use a FP unit for their wavelength calibration, e.g. CARMENES at Calar Alto (Quirrenbach et al., 2010), the Habitable Zone Planet Finder at the HET (Mahadevan et al., 2012), Espresso at the VLT (Mégevand et al., 2012), SPIRou at the CFHT (Artigau et al., 2011) and PEPSI at the LBT (Strassmeier et al., 2008).

The idea for this project has been influenced by both, the Laser Frequency comb (LFC), designed at the Max Planck Institute for Quantum Optics (MPQ), and the FP presented by Wildi et al. (2010) for the HARPS spectrograph. Designing and building a FP unit has been the first laboratory / instrumentation project of our group and while the lab already existed before, it has rarely been used, except for lab courses with the historic spectrograph (see ten Bruggencate and Jäger (1951) for the original setup and Dürbye (2013) for the current setup). Since no one with fiber knowledge was available, learning the basics of fiber optics and how they applied to FPs was the first step. The old spectrograph, while having a high resolution $(R=110,000)$, has been built for observing the spectrum of the sun. Therefore efficiency is not one of its prime features and it took quite a while to increase the coupling efficiency of the fibers to the point of acceptable signal to noise ratios in the final spectrum (see Fig. 1.4). Furthermore the spectrograph does not provide any wavelength calibration and is not stabilized with regard to pressure or temperature.

In early 2012 a FP test setup has been 

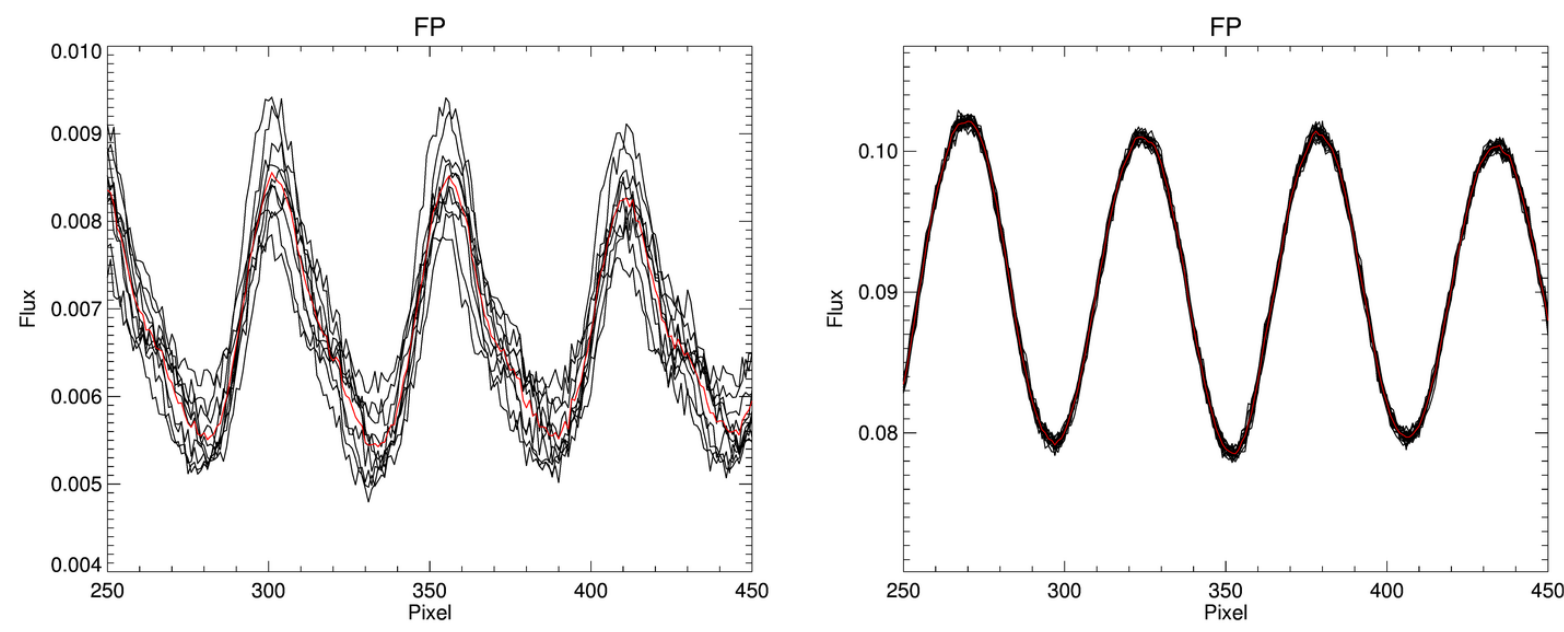

Figure 1.4: Measurements of the visual FP with the historic spectrograph with the improvements of the signal to noise ration of the FP due to increased coupling efficiency (from left to right). The red line is the mean spectrum of ten single exposures, shown in black.

taken to the Thüringer Landessternwarte Tautenburg to use their echelle spectrograph to measure a FP spectrum comparable to astronomical observations. Since the Tautenburg spectrograph is not fiber fed we improvised a fiber coupling and took spectra of the FP and a ThAr lamp. The results convincingly showed that the FP worked as intended and also illustrated the advantage of the FP compared to the ThAr lamp quite nicely (see Chapter 2, Fig. 2.2).

After some delays the arrival of the Fourier Transform Spectrometer (FTS) in Göttingen at the end of 2012 finally initiated high resolution measurements of the FP. The spectra with $\mathrm{R}=1,000,000$ showed details of the line profile which were not visible before (and have most likely not been observed at all for an astronomical FP before). For example the spectra clearly showed a huge difference in the line contrast as a function of fiber diameter and also showed asymmetric line profiles. Particularly the asymmetric line profiles have the potential to introduce problems for the wavelength calibration if they are time dependent. Simulations revealed the most likely cause for the observed effects: The fiber used to couple the light from the light source into the FP. Furthermore the simulations also helped predicting potential RV-errors due to the use of fibers in conjunction with FPs in general.

In parallel to both the simulations and the measurements the vacuum tank and its cooling concept has been designed and built in cooperation with the Zentralwerkstatt der Fakultät für Physik and Huber Kältemaschinenbau GmbH'. The final setup provides a temperature and pressure stability good enough to ensure a RV-stability of the FP of better than $10 \mathrm{~cm} \mathrm{~s}^{-1}$. However, in order to verify this RV stability in the lab, first the FTS has to be stabilized to the same order of magnitude. This is currently being worked on by our group.

Finally, in late 2013 we took the final setup to Tautenburg in order to connect the FP unit to the CARMENES calibration unit which is being built in Tautenburg. Different exposure times can't be tested sufficiently at a FTS due to its different functional principle compared to an echelle spectrograph. Thus with the Tautenburg spectrograph the exposure times for different fiber diameters and lamps could be tested in a setup very similar to CARMENES, 
using the light sources, the calibration unit and all fibers according to the CARMENES specifications. After further testing, the two FP units (one for the visual and one for the near infrared) will be used as wavelength references during observations in order to correct for instrumental drifts during the night of CARMENES. First light of the full instrument at Calar Alto is expected to occur in late 2014.

This work is structured in the following way: Chapter 2 describes how wavelength calibration is done in principle and explains different calibration sources in more detail.

Chapter 3 introduces the CARMENES project, giving an overview on the instrument, the planned survey and how the wavelength calibration will be applied.

Chapter 4 consists of my publication regarding the FP test setup for CARMENES (Schäfer and Reiners, 2012). It also explains in detail how parameters like pressure and temperature stability translate into the RV precision of the FP.

Chapter 5 explains additional effects with regard to the shape of the FP lines originating in the fibers used. It also deals with the possible RV errors which can be introduced by fiber scrambling effects. This Chapter is submitted for publication in A\&A (Schäfer et al., 2013).

Chapter 6 shows the final setup designed and built for the FP units for CARMENES. It describes the electronic hardware used, the vacuum tank, the cooling concept, the expected performance of the whole unit and shows the 'first light' results.

Chapter 7 explains the relationship between CARMENES and XSHOOTER and shows the first version of a spectral atlas of M-type stars for the wavelength range between $350 \mathrm{~nm}$ and $2450 \mathrm{~nm}$ made from XSHOOTER observations, including line identification and the removal of atmospheric lines.

Chapter 8 gives a summary on this work and also gives an outlook on the next steps with regard to the FP unit. 


\section{Chapter 2}

\section{Wavelength calibration}

The wavelength calibration of a spectrum is usually done as one of the final steps of the data reduction. For this purpose calibration lines are either inserted into the science spectra itself or are taken separately (either consecutively or in parallel). For each order of the spectrum these calibration lines are identified using a reference line list. Normally, these reference lists are made from laboratory measurements of the calibration sources, e.g. Lovis and Pepe (2007) for Thorium Argon (ThAr) emission lamps. Ideally these line lists contain the wavelength for every line with very high accuracy. Each of the identified lines is then fitted, e.g. with a Gaussian, in order to find the line center. Finally a polynomial fit is used to constrain the global wavelength solution, creating a dispersion relation between wavelength and pixel position on the detector. The whole process can be iterated several times in order to improve the wavelength solution by rejecting outliers and optimizing the fit.

However, the quality of the fit largely depends on the number of unblended reference lines and how exact their position is known from laboratory measurements (assuming they don't change over time). There are different sources for calibration lines available. For example the telluric absorption lines of Earth's atmosphere are visible in every spectrum taken with a ground based instrument. Unfortunately, these lines are weather dependent since they are mostly water lines and they only form in certain wavelength regions. Other calibra- tion sources have to be inserted in the light path in between telescope and spectrograph (e.g. Ammonia gas cells, see Bean et al. (2010)). The most accurate calibration sources are usually injected into the spectrograph parallel to the science observation, e.g. using a second fiber or taken before and after the science exposure if only one channel is available. The most established method is the so-called simultaneous Thorium reference method (Baranne et al., 1996), using a ThAr Hollow Cathode Lamp as reference. This chapter explains the functional principle of Hollow Cathode Lamps (Sec. 2.1) and introduces alternative calibration sources: The Laser Frequency Comb (Sec. 2.2) and Fabry-Pérot etalons (Sec. 2.3).

\subsection{Hollow Cathode Lamps}

In 1916 Friedrich Paschen published his work about the first hollow cathode discharge as a light source for spectroscopy (Paschen, 1916). Since then the concept of Hollow Cathode Lamps (HCL) has been constantly improved, mainly due to its application in atomic absorption spectroscopy (Walsh, 1956). Today HCLs consists of a sealed glass tubes with a fill gas at some low pressure inside, a metal cathode and a metal anode (see Fig. 2.1). A discharge in the gas is created by applying a voltage between the cathode and the anode. Thus material is sputtered from the cathode and the energy of these particles dissipates due to collisions with the gas. Eventually this leads to narrow emission lines of both the metal and 
the gas atoms emitting from the HCL.

ThAr is the combination used most in astronomical applications, mainly because Th only has a single isotope and zero nuclear spin and therefore no hyperfine structure signature. Lovis and Pepe (2007) show how RV-precisions down to the $20 \mathrm{~cm} \mathrm{~s}^{-1}$ level can be achieved with HARPS, using ThAr HLCs for wavelength calibration. Depending on the wavelength range of the spectrograph in question, other materials are investigated, e.g. UNe for the nearinfrared (see e.g. Redman et al. (2010, 2011)).

HCLs are commercially available, relatively cheap and are easy to use. Unfortunately, the emission lines of two lamps never look identical because of small deviations of the materials used or slightly different gas pressures. Additionally, the different lines of a HCL vary dramatically in intensity and are not at all equally spaced since the line position and intensity origin from the atomic structure of the materials used. Therefore the quality of the wavelength solution obtained by the HCL varies with wavelength: there are large differences between wavelength regions with many lines, few blends, no saturation of the detector and regions with only very few lines, a large fraction of blended lines and saturated lines. Figure 2.2 shows a small section of a CCD image of the spectrum of both a ThAr emission lamp and a Fabry-Pérot etalon, taken at the Tautenburg spectrograph which illustrates the differences in terms of line density and intensity variations. Finally, there are aging effects of HCLs, introducing additional problems for long term calibration because the internal pressure changes on timescales of months and therefore the position of some of the lines changes (Mayor et al., 2009).

\subsection{Laser Frequency Comb}

A Laser Frequency Comb (LFC) is a light source whose spectrum consists of a large number of equidistant lines, produced by a pulse train of a mode locked laser (see Hall and Hänsch (2005) for an introduction to LFCs). An example for the astronomical application of the LFC technology is the Astrocomb, developed by Wilken et al. (2012) for the HARPS spectrograph. Figure 2.3 shows a sketch of the setup. Wilken et al. use a Yb fiber laser, stabilized by a Rb atomic clock utilizing the Pound-Drever-Hall (PDH) locking scheme (see Drever et al. (1983)) to generate the comb. Unfortunately the distance in wavelength between the resulting peaks is far too small to be of any use in an astronomical spectrograph (about $250 \mathrm{MHz}$ or $\sim 3 \cdot 10^{-4} \mathrm{~nm}$ ). Therefore three Fabry-Pérot filter cavities are used to successively filter out most $(\sim 98 \%)$ of the peaks until the peak-to-peak distance is about $0.02 \mathrm{~nm}$ (18 GHz spacing). These filter cavities have also to be stabilized with a continuouswave laser (not shown in the figure), using the PDH locking scheme. A high-power amplifier is used to increase the power of the comb which then also has to be broadened by a photonic crystal fiber (PCF). Finally, the light is fed into a multimode fiber, scrambled and is coupled into the HARPS spectrograph. For a very detailed description of how the LFC works and its applications at the HARPS spectrograph see Wilken (2010).

The main feature of a LFC is its absolute calibration due to its stabilization to a $\mathrm{Rb}$ clock. Therefore the exact wavelength of every comb line is known to the precision of the PDH locking mechanism. This locking can be as good as $10^{-11}$ or better, depending on the quality of the hardware used (e.g. Wilken et al. (2012) showed a short time stability of $2.5 \mathrm{~cm} \mathrm{~s}^{-1}$ ). Consequently, the main problem of the LFC is its price tag: Buying an Astrocomb similar to the one used at HARPS with a stability of $10^{-11}$ costs about $300,000 €$ and at least in the nearer future it won't be a turn-key system but will need substantial maintenance. Also, currently LFCs only work with quite narrow bandwidth (a few hundred $\mathrm{nm}$ ) and only at certain wavelengths (around multiples of the lasers original wavelength). While those limitations might be overcome at some point, at present they 


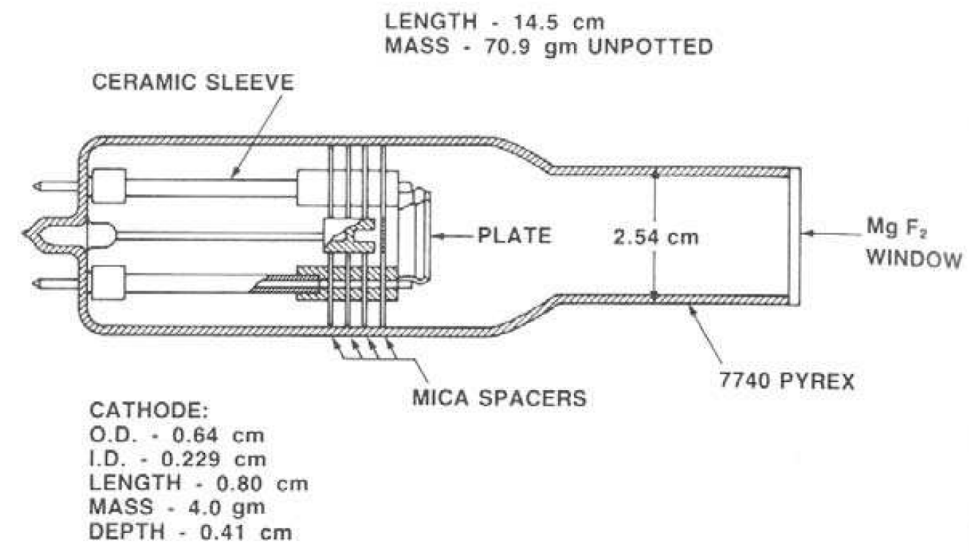

Figure 2.1: Schematic design of a hollow cathode lamp. Source: Klose and Bridges (1987)

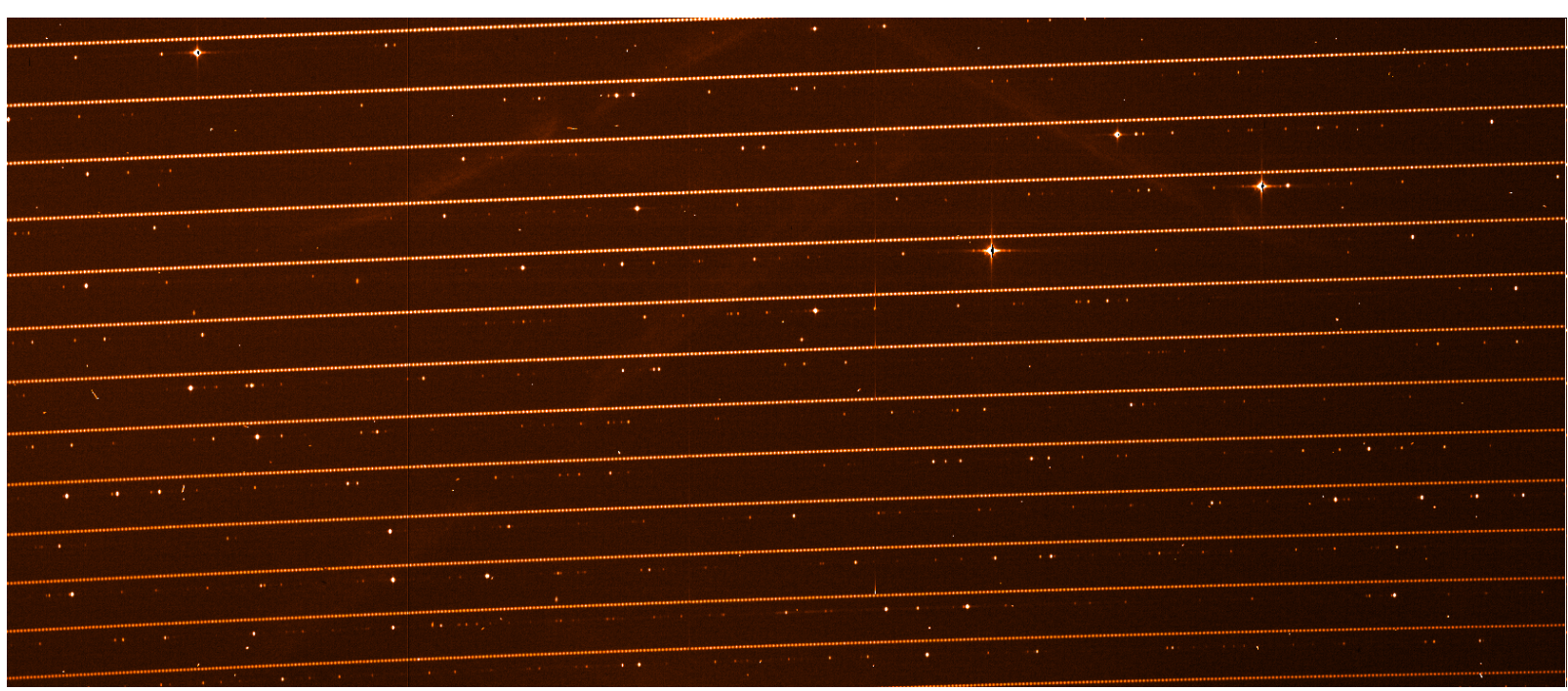

Figure 2.2: Small section of a CCD image of the spectrum of both a ThAr emission lamp and a Fabry-Pérot etalon with an artificial offset of 10 pixels.

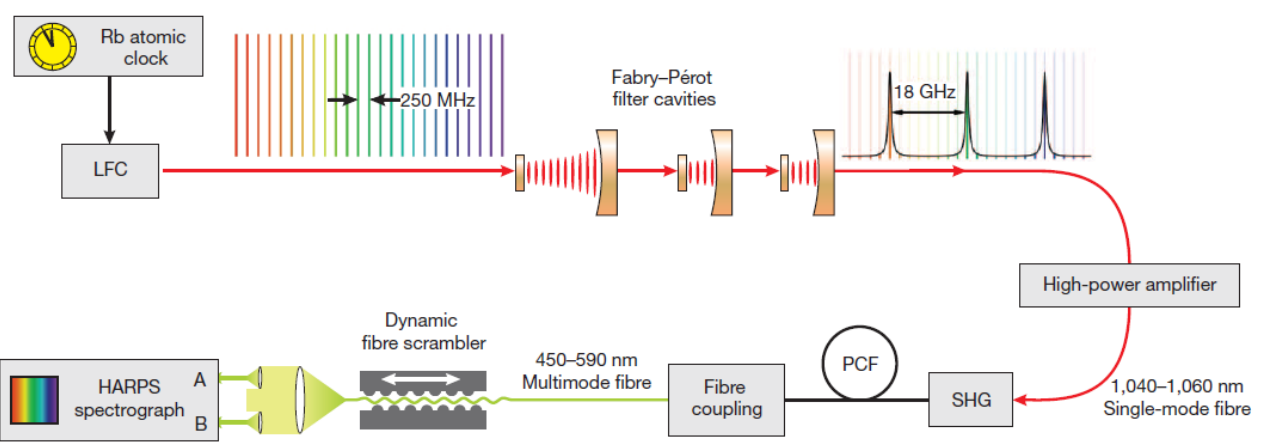

Figure 2.3: Sketch of the LFC setup tested at HARPS. Source: Nature (Wilken et al., 2012). 
make the LFC an unrealistic option for most astronomical spectrographs.

\subsection{Fabry-Pérot etalon}

Wildi et al. (2010) first showed how a FabryPérot etalon (FP) could be built as a tool for high precision wavelength calibration. The basic design consists of two flat, soft coated, semitransparent mirrors, held in place by a Zerodur spacer. The light is fed in on one side and exits on the other side, while the FP imprints its transmission signature onto the spectrum of the originally black body spectrum of the light source. The peaks created by the interference inside the FP are equally spaced (in frequency), their shape depends mainly on the reflectivity of the mirrors and the peak-to-peak distance is a function of the distance between the two mirrors. In principle, this can be used at all wavelengths as long as a suitable mirror coating is used and a light source with enough flux in that particular wavelength range is available.

The stability of the peaks depends on the stability of the true mirror distance (influenced by the index of refraction and the expansion coefficient of the Zerodur spacer) which in turn depends on external parameters like temperature stability, pressure stability and stability of the coupling devices. Wildi et al. reached a stability of $10 \mathrm{cms}^{-1}$ during one night while in this work the goal is to design and built a FP unit with stability of $10 \mathrm{~cm} \mathrm{~s}^{-1}$ over weeks, months or even years.

The main selling point of a FP is its technical simplicity and its easy adaption to any wavelength region. The device itself is relatively cheap $(\sim 2500 €)$ while the challenging and costly part is the temperature and pressure stabilization. Still, the total cost of a FP unit with a stabilization of $10 \mathrm{~cm} \mathrm{~s}^{-1}$ will be at least a factor of 10-20 cheaper than a LFC while covering a much larger wavelength range without the need for intensive maintenance. The downside is, that a FP by itself cannot provide an absolute wavelength cali- bration. Because the true distance between the mirrors is unknown, the absolute position of each peak, while stable with time, is unknown. Therefore an additional reference point is needed in order to create a wavelength solution, e.g. a ThAr spectrum or a LFC. 


\section{Chapter 3}

\section{CARMENES}

CARMENES (Calar Alto high-Resolution search for M dwarfs with Exo-earths with Nearinfrared and optical Echelle Spectrographs) is a next-generation instrument which is currently being built for the $3.5 \mathrm{~m}$ telescope at the Calar Alto Observatory. The project is realized by a consortium of eleven German and Spanish institutions with the goal to perfrom a five-year exoplanet survey on $300 \mathrm{M}$ stars. In order to cover the full wavelength range between 0.55 and $1.7 \mu \mathrm{m}$ the instrument will have two separate spectrographs, one for the visible part and one for the near infrared part of the spectrum. Both spectrographs will be fiber fed and provide a spectral resolution of $\mathrm{R}=82,000$. In order to achieve a radial velocity precision of $1 \mathrm{~m} \mathrm{~s}^{-1}$ both instruments are located inside temperature-stabilized vacuum tanks.

CARMENES has been optimized for the search for Earth-like planets inside the habitable zone of low-mass stars. Using the radialvelocity method it will provide a better sensitivity for the detection of these planets than any comparable instrument because of its combination of optical and near-infrared spectrographs. The simultaneous observation with both spectrographs will also be extremely helpful in order to distinguish between actual planets and false positives caused by e.g. magnetic activity of the host star.

\subsection{The survey}

The main scientific goal of CARMENES is to carry out a survey of M-type stars in order to characterize their planetary population, including the detection of low-mass planets within the habitable zones of these stars. The main focus will lay on very cool stars with spectral type M4V or later which are only moderately active. The long-term radial velocity stability of $1 \mathrm{~m} \mathrm{~s}^{-1}$ will allow the detection of e.g. a $2 \mathrm{M}_{\text {Earth }}$ planet inside the habitable zone of an M5V star. For M-type stars near the hydrogen-burning limit even an earth-mass planet could be detected inside the habitable zone.

The CARMENES survey will include 300 M-type stars. With a number this large the program will provide valuable statistics on the population of planets around this type of stars, such as their frequency, orbital parameters and mass. Another question which will be answered by this survey is whether or not the low rate of Jovian planets around M stars is real or not. Since most of the targets will be within $20 \mathrm{pc}$ the newly detected planets will be feasible targets for further characterization, e.g. for analyzing the chemical composition of their atmospheres.

\subsection{The instrument}

The efficiency of CARMENES has been optimized for the $\mathrm{Y}$ band (around $1000 \mathrm{~nm}$ ) since this is the most important wavelength region to measure radial velocities in mid to late M- 


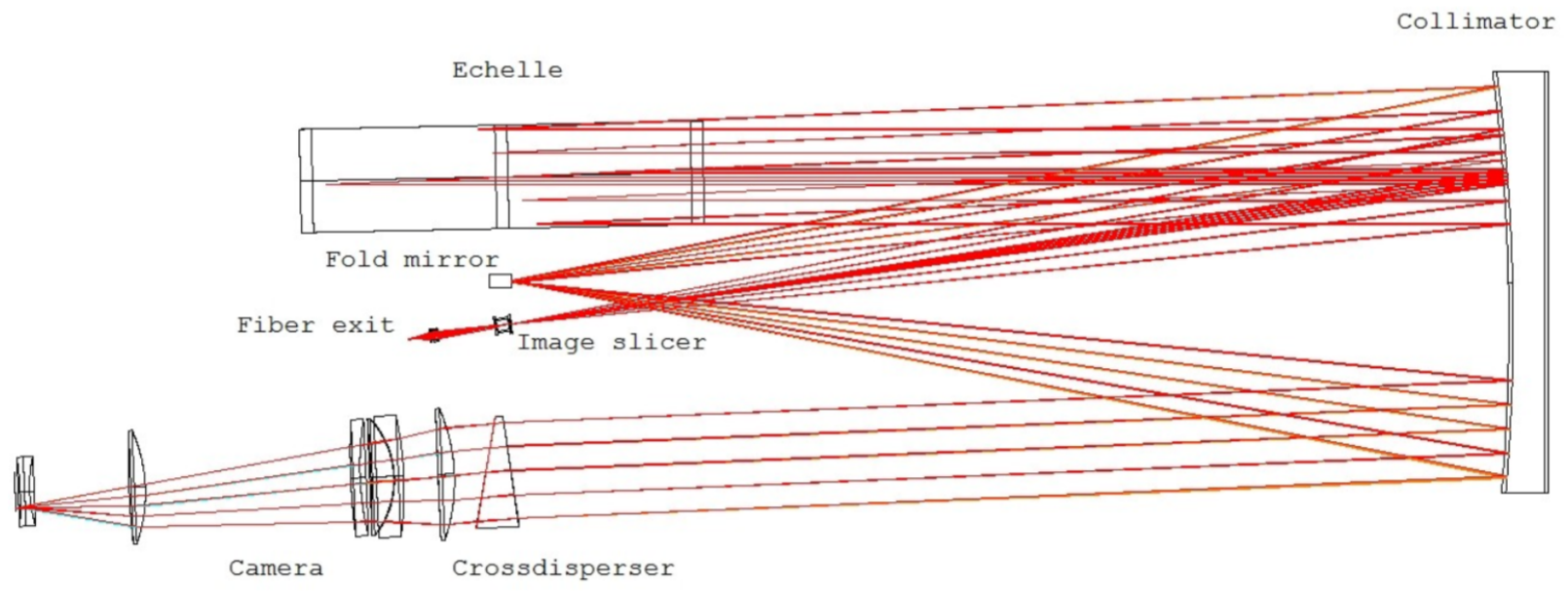

$\mathfrak{x}^{\mathrm{x}}$

Figure 3.1: Layout of the VIS channel optical design (source: CARMENES optics Final Design Review document FDR-02A, VIS channel optics design).

type stars. Due to the Si cutoff at $1100 \mathrm{~nm}$ and the poor performance of CCDs around $1000 \mathrm{~nm}$ in general, a near-infrared detector is required. Consequently CARMENES will have a CCD for the wavelength region between 550 and $1050 \mathrm{~nm}$ and a $\mathrm{HgCdTe}$ detector for the region between 900 and $1700 \mathrm{mn}$ respectively.

A dichroic beamsplitter is placed in the front end to divide the light coming from the telescope into two separate fibers, one for each spectrograph. Both spectrographs are mounted on optical benches inside vacuum tanks, sitting in the coudé room of the telescope. The room itself is a climatic chamber to keep the room temperature stable within $\pm 0.1 \mathrm{~K}$. This translates to a temperature stability within the vacuum tanks of $\pm 0.01 \mathrm{~K}$ over $24 \mathrm{~h}$. Additionally, the near-infrared spectrograph will be cooled down to $140 \mathrm{~K}$ using liquid nitrogen inside a radiation shield within the tank.

Both spectrographs have very similar layouts, using a cross-dispersed echelle in quasiLittrow mode with a white pupil design. The dispersion is done with a $2 \times \mathrm{R} 4$ mosaic grating while a grism is used to cross-disperse the orders. The near-infrared detector will be a mosaic of HAWAII-2RG detectors, the visible spectrograph will use an e2v CCD231-84. Figure 3.1 shows the setup of the VIS channel.

There are two fibers for each spectrograph leading from the front-end of the telescope to the instrument. The baseline strategy of observation is to inject starlight in one fiber and light from a calibration source into the other. However, other modes such as having both fibers fed with light from the star or star + sky background are also available. Both spectrographs have a separate calibration unit which provides light from different calibration sources that can be chosen with the help of a rotating mirror.

\subsection{Wavelength calibration with CARMENES}

The wavelength calibration strategy for CARMENES will be to use both, HCLs and a Fabry-Pérot etalon. A set of three masterlamps (ThNe for the visual channel and $\mathrm{UNe}$ for the near-infrared channel) will be used to produce the wavelength solution. One lamp will be used daily, one lamp will be used once 
per week and one will only be used once per month. Comparing these spectra will show any systematic offsets between the lamps. The wavelength solution produced every day will be checked against a reference wavelength solution made with a set of three super-master lamps twice a year to check for long term drifts.

Each day during daytime the FP spectrum will be used to interpolate the wavelength solution of the master lamps (benefiting from the much higher line density of the FP). During the night the FP will always be fed into the calibration fiber of the spectrograph in order to trace the differential nightly drift of the instrument. If the FP proves its stability over longer periods of time (days, weeks, months, ...) the cross calibration against the master lamps will be done less frequently.

There are a number of advantages using this concept: The number of HCLs needed for operation is greatly reduced. Normally there would be a set of slave lamps to be used during the science observations instead of the FP. Due to the large number of hours of operation these lamps would fail quite rapidly (expected life time of two month) and would need constant checking against the master lamps. Additionally, using the FP to interpolate the wavelength solution provides a much better result for wavelength regions which otherwise would not have many lines to be used. Finally, the FP might also be useful to identify discontinuities on the detector pixel grid. 


\section{Chapter 4}

\section{Fabry-Pérot Etalon: Simulations \& test setup}

This Chapter introduces the basic equations of a Fabry-Pérot Etalon (FP). The simulations shown here describe the influence of the different external parameters to the resulting spectrum and thus set the stability requirements of these parameters for a given RV-precision (Sec. 4.1). Section 4.2 explains the FP setup used for all laboratory measurements up to the completion of the vacuum tank (see Chapter 6). Section 4.3 shows the first light observations done at the Thüringer Landessternwarte Tautenburg and Sec. 4.4 concludes this chapter.

All sections are published in Schäfer and Reiners (2012) in the Society of Photo-Optical Instrumentation Engineers (SPIE) Conference Series 2012 as 'Two Fabry-Perot interferometers for high precision wavelength calibration in the near-infrared'.

\subsection{Simulations}

The transmission function of a Fabry-Perot interferometer (FP) can be written as

$$
I=\frac{1}{1+F \sin ^{2}\left(\frac{\delta}{2}\right)},
$$

with the coefficient of finesse $F$ and the phase shift $\delta$ :

$$
\delta=\frac{1}{\lambda} 4 \pi n l \cos (\theta) .
$$

$\lambda$ is the wavelength, $n$ the refraction index of air inside the FP, $l$ is the distance of the mir- rors and $\theta$ the angle of the passing ray of light. Figure 4.1 shows the transmission function for an ideal FP while in Fig. 4.2 the transmission function is broadened and rebinned according to the CARMENES specifications.

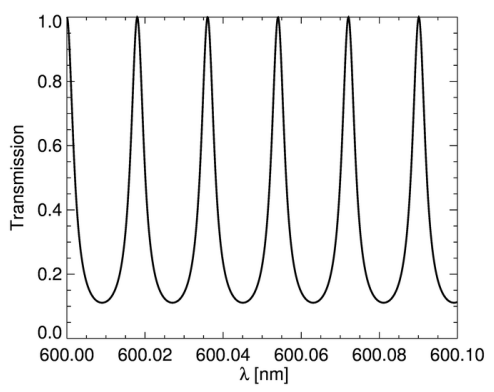

Figure 4.1: Transmission function of a theoretical FP with $F=8, l=$ $9.99 \mathrm{~mm}, n=1$ and $\theta=0^{\circ}$.

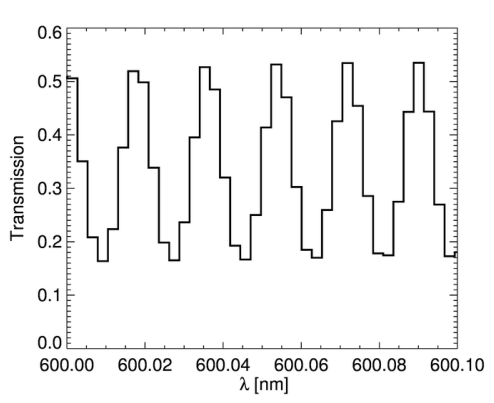

Figure 4.2: The same FP as in Fig. 4.1 but with the resolution and pixel sampling of CARMENES $(\mathrm{R}=82,000)$. 
The position of the peaks can be derived by looking for the maxima of Eq. 4.1. This is the case for $\frac{\delta}{2}=0$, therefore the peaks can be found at

$$
\frac{2}{\lambda} n l \cos (\theta)=N
$$

where $N$ is an integer number. From this one can derive the radial velocity error introduced by the FP:

$$
\frac{\partial v}{c} \sim \frac{\partial \lambda}{\lambda} \sim \frac{\partial x}{x}
$$

where $x$ can either be $n, l$ or $\cos (\theta)$. The stability requirements for each of these parameters for a given RV-precision can be calculated accordingly.

\subsubsection{Stability of the mirror distance $l$}

The FP itself is made of Schott Zerodur in order to minimize thermal expansion and therefore deviations of the mirror distance. Schott Zerodur has an coefficient of expansion of 2 . $10^{-8} \mathrm{~K}^{-1}$. As shown in Fig. 4.3 the temperature has to be stabilized to $0.018 \mathrm{~K}$ in order to reach a RV-precision of $10 \mathrm{~cm} \mathrm{~s}^{-1}$.

\subsubsection{Stability of the angle $\theta$}

The stability requirements for the angle of the light passing the FP are shown in Fig. 4.4. Achieving an angle stability of $0.0015^{\circ}$ implies two problems:

- The mechanical stability of the setup

- Stability of the light output of the fiber

The mechanical stability can be reached by using either by using a very stiff cage system or by including the fiber-lens system within the Zerodur structure.

However, using fibers to feed the white light into the FP introduces another source of error: Multimode fibers, which are the best option for white light, have many different modes which are carrying the light inside the

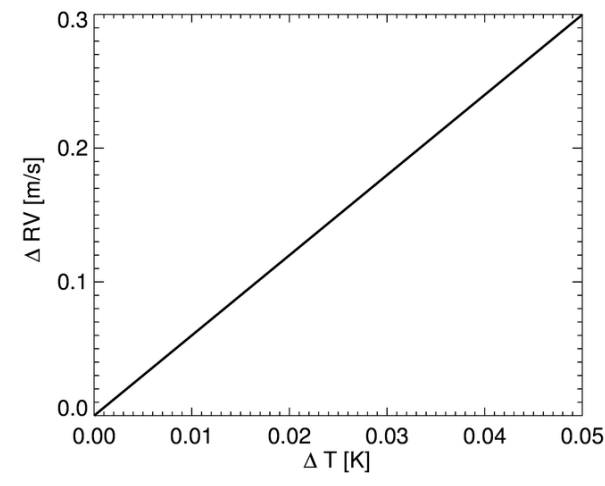

Figure 4.3: RV-precision in $\mathrm{m} \mathrm{s}^{-1}$ as function of temperature stability with respect to thermal expansion.

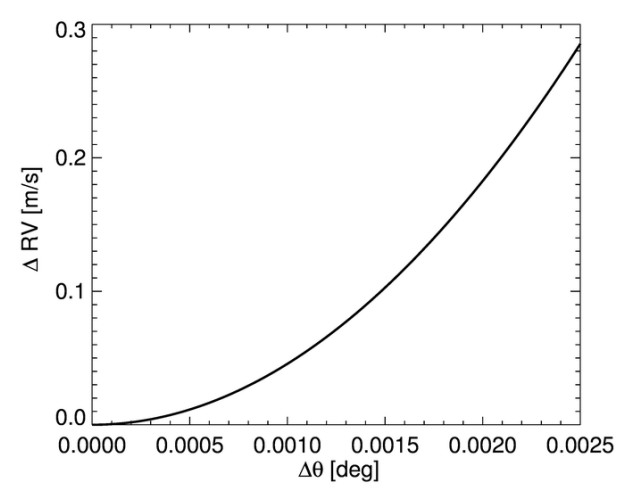

Figure 4.4: RV-precision in $\mathrm{m} \mathrm{s}^{-1}$ as function of angle stability.

fiber. Moving the fiber or putting stress on it, changes the modes used. This leads to a nonstable output at the exit of the fiber, more precisely the spot at the fiber exit changes both shape and position. Usually fiber scramblers of different kinds are used to resolve this issue for astrophysical applications. They mix the modes carrying the light inside the fibers and therefore produce a more stable output. However, since we are using a very bright light source there is also the option of using very small multimode fibers or even single mode fibers in combination with parabolic mirrors in order to reduce the angle deviation ${ }^{1}$.

1 see Chapter 5 for a detailed discussion on the influence of the fibers 

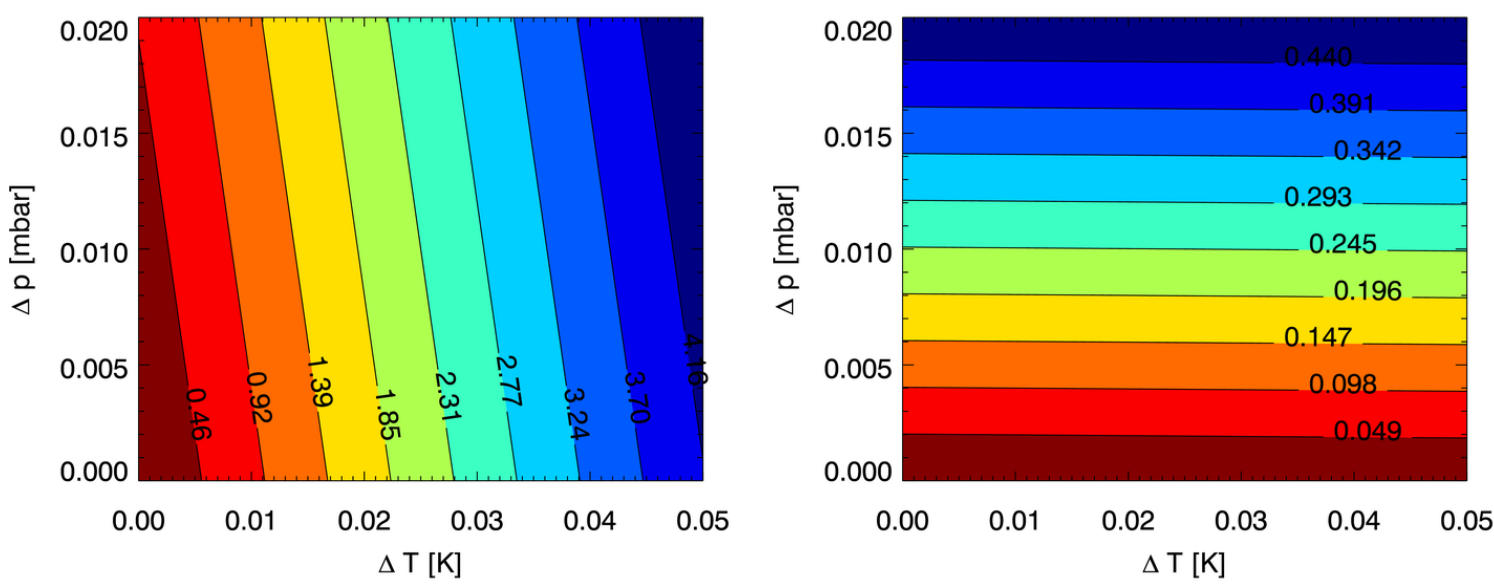

Figure 4.5: Left: RV-deviation in $\mathrm{m} \mathrm{s}^{-1}$ at $\lambda=900 \mathrm{~nm}, \mathrm{p}=1$ bar and $\mathrm{T}=20^{\circ} \mathrm{C}$ due to changes in pressure and temperature. Note the temperature scale is the same as for the thermal expansion (Figure 4.3). Right: Same plot but with $\mathrm{p}=1$ mbar.

\subsubsection{Stability of the index of re- fraction $n$}

As shown by Owens (1967) the refractive index of dry air is a function of the temperature $T$ and the pressure $p$. Using Eq. 4.4 and Owens calculations one can derive the resulting RV-precision for both different temperature and pressure-stability at different pressures.

As shown in Fig. 4.5, evacuating the FP to a pressure of 1 mbar not only decreases the RV-error due to a more stable index of refraction but also reduces the dependency on temperature deviations significantly. In order to reach a RV-precision of $10 \mathrm{~cm} \mathrm{~s}^{-1}$ one has to stabilize the pressure inside the FP stable to $\sim 4 \cdot 10^{-3}$ mbar while the temperature stability is much less critical than in the case of thermal expansion (see Fig. 4.3).

\subsection{Design}

\subsubsection{Optimization for CARMENES}

Our two FPs are designed for the infrared CARMENES spectrograph, which is currently being built for the Calar Alto $3.5 \mathrm{~m}$ telescope. The spectrograph will have two arms with a spectral resolving power of $\mathrm{R}=82,000$ : one for the optical (550-1050 nm) and one for the near infrared (900-1700 nm).

As shown by Butler et al. (1996), the intrinsic Doppler error in any portion of the spectrum due to photon-limited errors can be written as

$$
\sigma_{V}=\frac{1}{\sqrt{\sum\left(\frac{d I / d V}{\epsilon_{I}}\right)^{2}}}
$$

where $d I / d V$ is the local slope of the spectrum and $\epsilon_{I}$ is the uncertainty in the residual intensity at pixel i. In order to minimize this error one has to maximize the sum of all $d I / d V$ which, in the case of a Fabry-Perot spectrum, depends on the distance between the FP's mirrors. The optimal spacing is different for the two arms of CARMENES, as shown in Fig. (4.6). The resulting distance between neighboring peaks for both FPs is about $0.03 \mathrm{~nm}$.

\subsubsection{Test setup}

As illustrated in Figs. 4.7 and 4.8, the FP is mounted inside a cage construction and will be fiber-fed with white light from an external light source. 


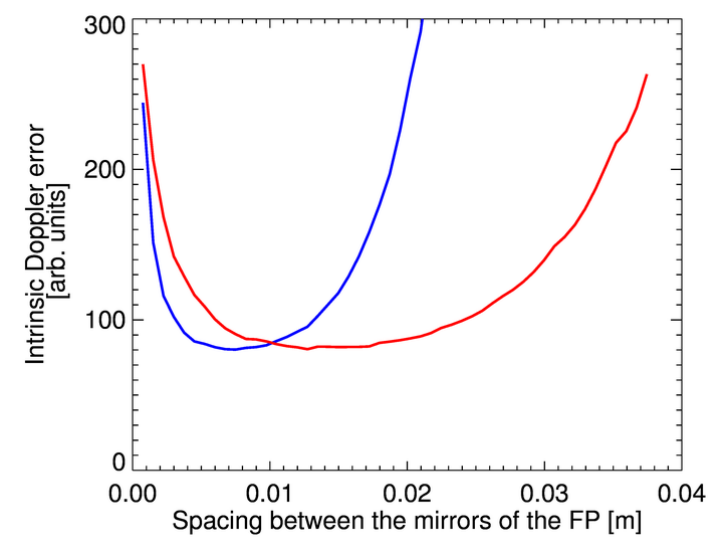

Figure 4.6: The intrinsic Doppler error as a function of the FP's spacing. The minimum and therefore the optimal value is different for the two arms of CARMENES. Solid line: optical arm, dashed line: NIR arm.

As a light source, a $10 \mathrm{~W}$ Halogen lamp is used. It covers the full wavelength range of both arms with sufficient flux - since the optical arm starts at $600 \mathrm{~nm}$ the limited flux of Halogen lamps in the blue won't cause problems for the setup. The light from the Halogen lamp is coupled into a multimode fiber $(10 \mu \mathrm{m}$ diameter, see subsection 4.1.2). A parabolic mirror is used to collimate the light from the fiber and sends it through the FP. A similar mirror on the other side of the FP is used to couple the light into the output fiber, which leads to the calibration unit. From there, the light goes to the front end and finally into the spectrograph. The whole FP-unit will be placed inside a vacuum tank, which will provide the temperature and pressure stability needed. A summary of the requirements for the FP-unit can be found in Table (4.1).

\subsection{First light}

The optical Fabry-Perot was tested at the coude echelle spectrograph at the Landessternwarte Tautenburg, which has a spectral resolution of up to $\mathrm{R}=67,000$. Usually the spectrograph is not fiber fed so an injection stage to use the light from the output fiber had been built. Since the FP-unit is not yet inside a vacuum tank and therefore has no temperature and pressure stabilization one can expect the RVprecision of the FP to be of the order of $100 \mathrm{~m} \mathrm{~s}^{-1}$. Once the vacuum tank is built we will test the FP's stability on different timescales with a Fourier Transform Spectrograph. Figure 4.9 shows parts of the spectra of both, the FP and a ThAr lamp.

\subsection{Conclusions}

We have designed and built two Fabry-Perot calibration units, each of them optimized for one of the two arms of the upcoming CARMENES spectrograph. They provide thousands of spectral lines with a much more homogeneous distribution and intensity than current hollow cathode lamps (e.g. ThAr). We have shown the stability requirements for the different parameters needed to reach an internal RV stability of $10 \mathrm{~cm} / \mathrm{s}$. In order to reach these requirements we will put the calibration systems into vacuum tanks. Designing and building these tanks will be the next step. Finally we will test the RV stability using a Fourier Transform Spectrograph in our lab. 


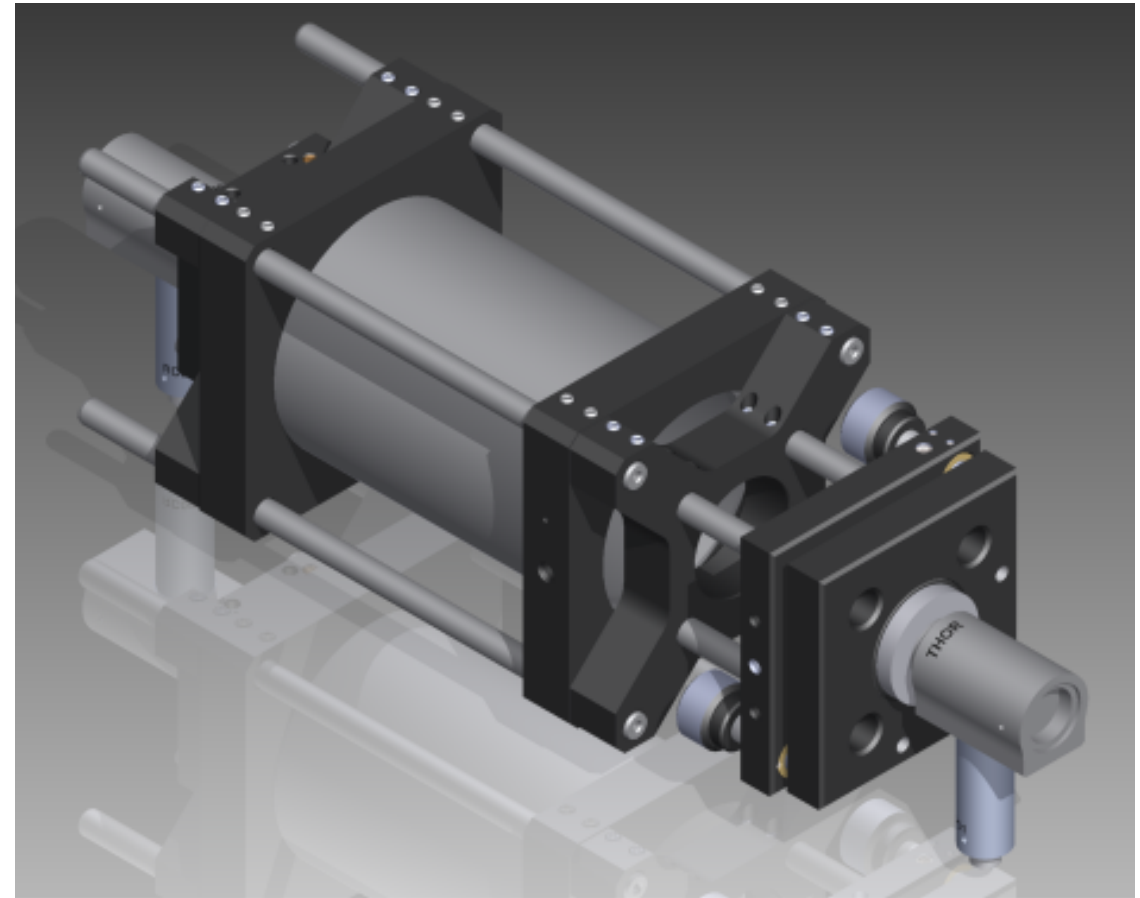

Figure 4.7: 3D image of the FP-unit. The FP itself is located inside the lens tube, which is mounted in a cage construction.
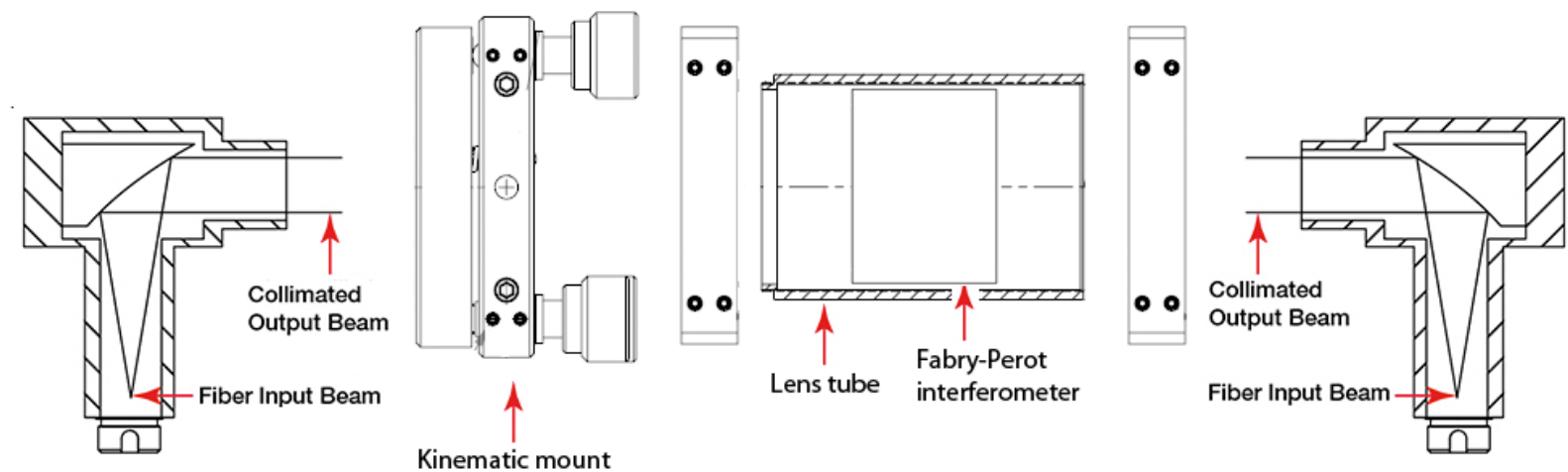

Figure 4.8: Sketch of the FP-unit.

\begin{tabular}{ll}
\hline Parameter & Requirement \\
\hline Temperature stability & $0.018 \mathrm{~K}$ \\
Pressure stability & $4 \cdot 10^{-3}$ mbar \\
Angle stability & $0.0015^{\circ}$ \\
Spectral coverage & $\mathrm{FP} 1: 600-1050 \mathrm{~nm}$ \\
& $\mathrm{FP} 2: 900-1350 \mathrm{~nm}$ \\
Average distance between peaks & $0.03 \mathrm{~nm}$ \\
RV stability & $10 \mathrm{~cm} \mathrm{~s}^{-1}$ \\
\hline
\end{tabular}

Table 4.1: System parameters. 


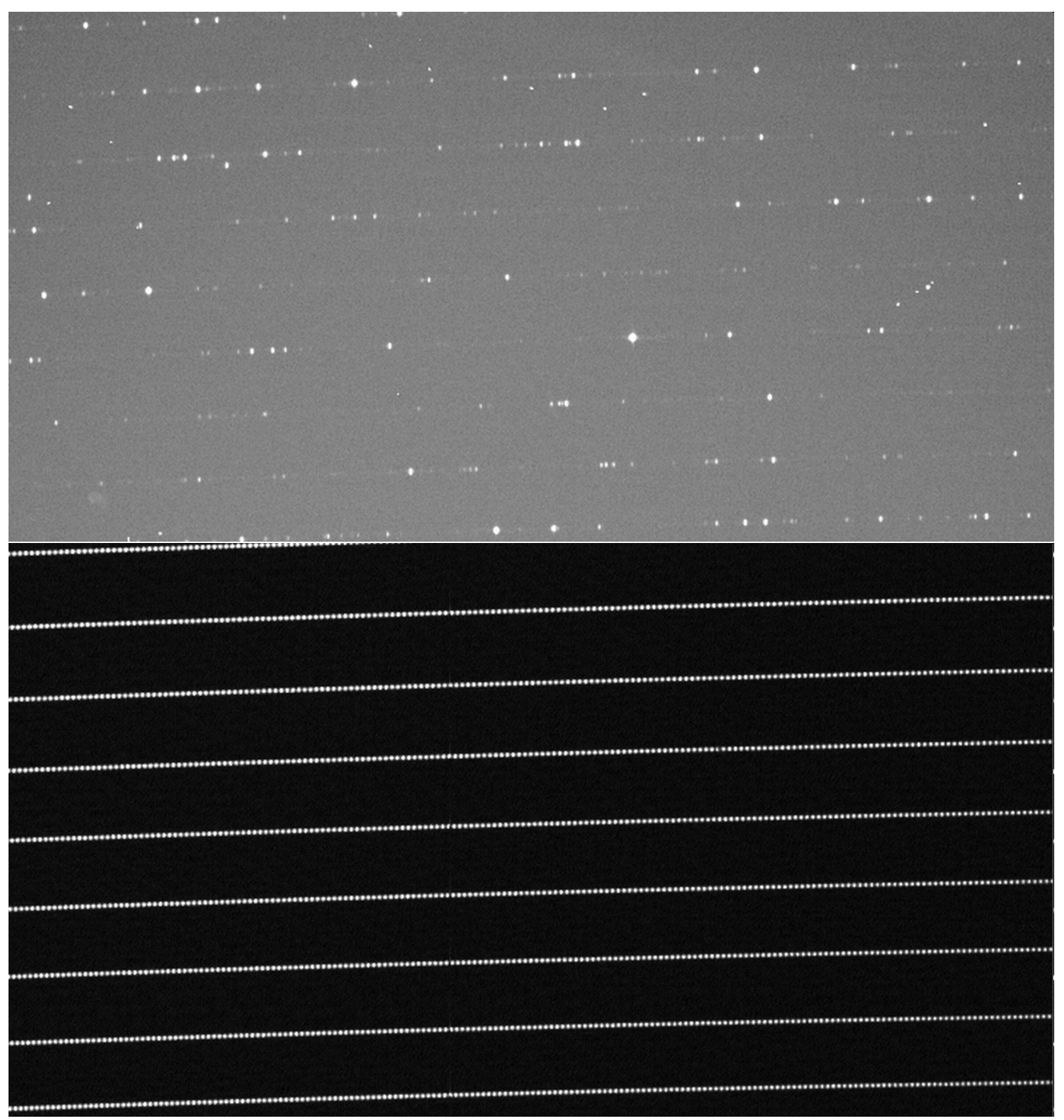

Figure 4.9: Raw frames of the Tautenburg echelle spectrograph. Parts of the spectra of both the ThAr-lamp (top) and the Fabry-Perot (bottom) are shown to illustrate the difference in line density and brightness homogeneity. 


\section{Chapter 5}

\section{Fabry-Pérot Etalon: Fibers and their illumination}

This Chapter explains what kind of effects the fibers and their illumination have on the FP spectrum. As shown in the following sections, fibers not only influence the shape of the FP lines (Sec. 5.2 and 5.3) but they can also introduce RV shifts due to scrambling effects (Sect. 5.4). These potential RV shifts need to be taken into account in addition to the influence of temperature and pressure (see Chapter 4).

All sections from this Chapter are taken from Schäfer et al. (2013) which is submitted for publication in Astronomy \& Astrophysics as 'The influence of fibers and their illumination on the radial velocity stability of FabryPerot etalons'.

\subsection{Motivation $^{1}$}

The requirements of a FP unit with respect to temperature and pressure stabilization have been investigated before (Wildi et al., 2010; Schäfer and Reiners, 2012). While challenging, it is possible to decrease the RV-errors, occurring due to changes of the temperature and pressure down to a stability of $10 \mathrm{~cm} \mathrm{~s}^{-1}$ by using a vacuum tank and a very sophisticated temperature stabilization. However, at this level of RV-precision additional effects related to the fibers become important. Investigating these effects in the actual spec-

1 In Schäfer et al. (2013) this is part of Sect.1 (Introduction) tra requires extremely high spectral resolving power, hence they usually remain undetected in astronomical spectrographs. Nevertheless, they influence the RV-performance of the FP unit significantly. Measurements with our Fourier Transform Spectrometer (FTS) show line shifts, asymmetries and contrast changes of the FP signal, caused by the fiber used for coupling the light into the FP. We are able to reproduce these effects by simulating the distribution of rays crossing the FP and investigated how this introduces RV-shifts of the FP peaks. Our simulations also show that changes in the light distribution on the fiber head in front of the FP can lead to substantial RV-deviations of the order of several $\mathrm{m} \mathrm{s}^{-1}$ that scale with the diameter of the fibers used.

\subsection{A real Fabry-Pérot}

The standard way to set up a FP for wavelength calibration is to use a fiber to feed light from a broadband light source into the FP (fiber A) and to couple the throughput into another fiber, leading to the spectrograph (fiber B). Figure 5.1 shows a schematic overview of such a setup, which makes it quite easy to use a vacuum tank or any sort of shell structure in order to achieve the desired stability requirements (temperature and / or pressure) since the fibers can be fed through vacuum flanges.

The light coming from the broadband light 


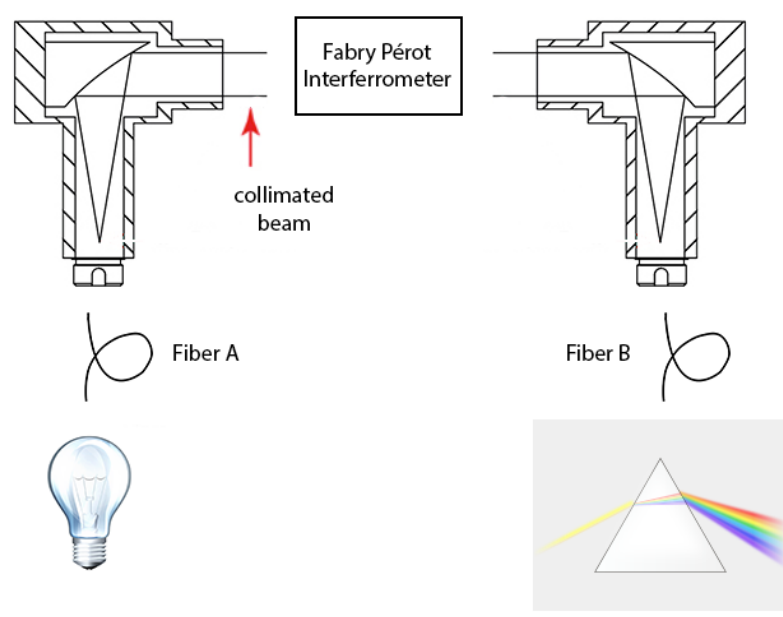

Figure 5.1: Schematic overview of the test setup.

source will exit fiber A within a cone, given by the numerical aperture (NA) of the fiber. A very efficient way to collimate the light is to use a parabolic mirror to avoid chromatic or spherical aberration, but the same considerations apply if lenses are used. An ideal point source placed in the focal point of that mirror would produce a perfectly collimated beam of light. However, a fiber is spatially extended and one can calculate the deviation from the parallel beam for each ray exiting the fiber head via

$$
\theta_{\text {dev }}=\arctan \left(\frac{D}{f}\right)
$$

with $D$ being the distance of the ray from the fiber midpoint and $\mathrm{f}$ being the effective focal length of the parabolic mirror. As long as the parabolic mirror is perfectly aligned, the angle $\theta$ of a single ray of light crossing the FP only depends on the position of its origin at the fiber exit. Rays coming from the center of the fiber will cross the FP with $\theta=0^{\circ}$ while all other rays will have an angle corresponding to the distance between the exit point of the ray and the fiber midpoint. The rays with $\theta \neq 0^{\circ}$ see a slightly different mirror distance $l$ and their interference peaks are shifted with respect to $\theta=0^{\circ}$ rays. All rays originating at a certain distance from the fiber midpoint will result in the same angle $\theta$ and all points on concentric circles of a certain radius around the midpoint will result in the same FP spectrum. In order to create the total spectrum of all rays, we need to know the 'weight' of each of these annuli, e.g. what percentage of the total light crosses the FP with $\theta=0.1^{\circ}$. One can calculate how much each annulus contributes to the total transmission spectrum of the FP by calculating its area:

$$
A=\pi\left(R^{2}-r^{2}\right)
$$

with $R$ and $r$ corresponding to the outer and the inner radius of the annulus, respectively. We can also introduce inhomogeneous light distributions e.g. a Gaussian profile (as long as the profile is symmetric). Using this weighting, the total spectrum of the system fiber + collimator + FP can be calculated by adding up the FP spectra for all $\theta$ accordingly. Figure 5.2 shows the resulting spectra for fiber diameters of 50, 200, 400 and $900 \mu \mathrm{m}$ using a Gaussian light distribution with a FWHM of $1 / 4$ of the fiber diameter and the perimeters of their illuminated areas on the fiber head. We can identify three main features in the spectra: (I) The peak position is blue-shifted for larger fibers, (II) the contrast decreases with increasing fiber diameter, and (III) the peaks become more asymmetric for larger fibers.

The blue-shift can be explained by looking at Eq. 4.3. As shown before, for a single ray the position of each peak can be described by

$$
\lambda_{\text {max }}=\frac{2 n l}{N} \cos (\theta) .
$$

Because of the cosine, increasing the angle $\theta$ from $\theta=0$ to $\theta>0$ while keeping $n$ and $l$ constant always shifts the peak positions towards smaller wavelengths. Since the angle $\theta$ depends on the position of the light ray exiting the fiber head, all points with a distance $r>0$ from the midpoint are blue-shifted with respect to the ones with $r=0$. Larger fibers will allow larger values of $r$ that are reflected 

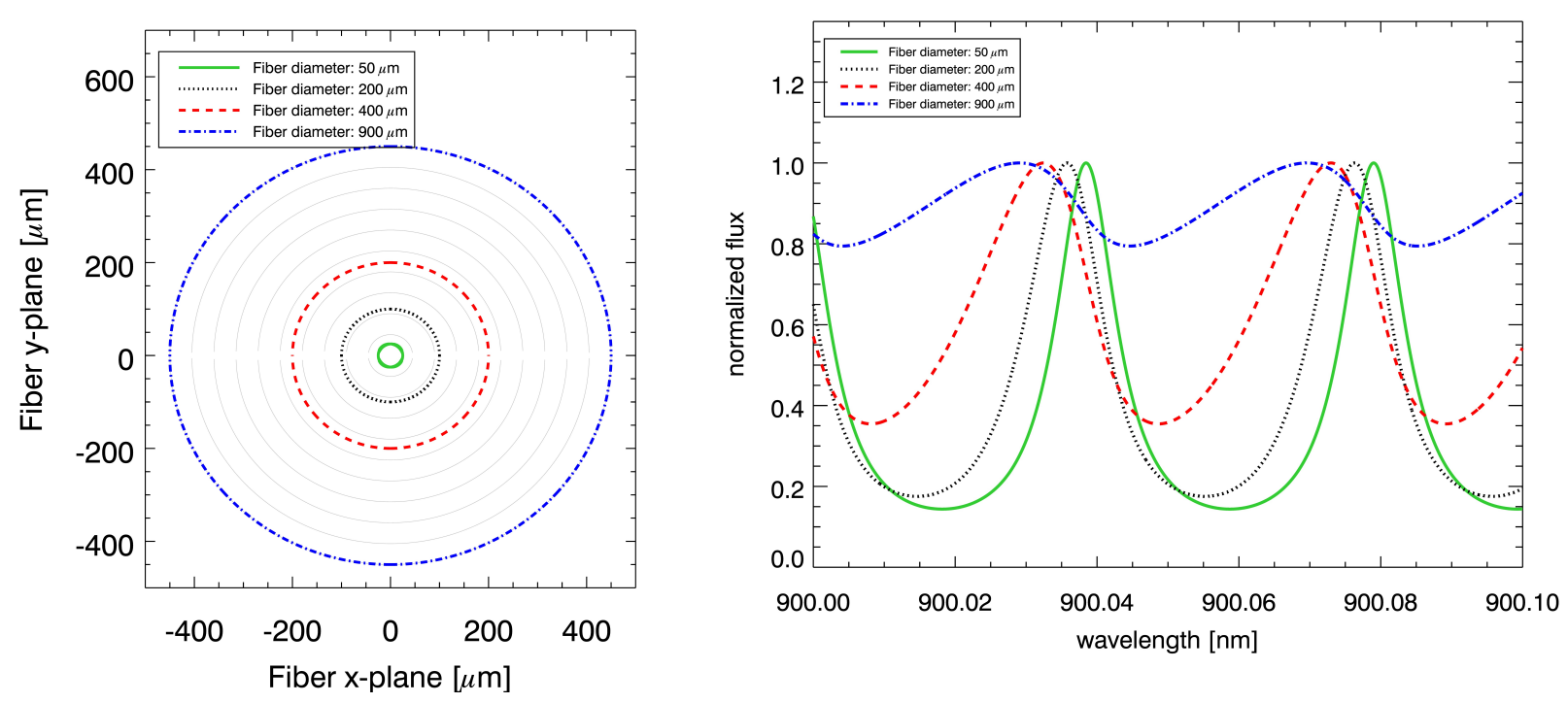

Figure 5.2: Left: Perimeter of the illuminated area for different fiber diameters. Right: Simulation of transmission spectra of a Fabry-Pérot for different fiber diameters assuming a Gaussian light distribution on the fiber.

into the FP under larger values of $\theta$, thus they will bear a stronger blue-shift compared to smaller fibers. At the same time this means their flux is distributed over a large range of $\theta$, and adding up all the spectra according to the weighting distribution (see Eq. 5.2) leads to a diminished contrast. Therefore, using larger fibers will not increase the FP contrast in the final spectrum even though the total flux is increased. On the contrary, smaller fibers will have a much better signal to noise as long as one can increase the exposure time accordingly.

Equation 5.2 also shows that the outer areas of the fiber head contribute much more strongly to the final spectrum compared to the areas in the center. In combination with the contrast effect, this leads to asymmetric lines with a tail towards the blue. Thus, larger fibers will not only have a reduced contrast, the peaks also become more asymmetric compared to small fibers. The transmission spectrum from one FP fed with different fibers can therefore show shifted peak positions and different line shapes.

Similarly, ring-like structures of the light distribution will shift the peak positions, because they mimic different fiber sizes while having less flux in the center. Such features can appear even in octagonal fibers (e.g. shown by Chazelas et al., 2010, Fig. 6). Compared with uniform light distributions this introduces shifts of several hundreds or even thousands of $\mathrm{m} \mathrm{s}^{-1}$. Particularly in large fibers these rings could have a great impact on the RV-stability, whereas small fibers would be affected to a much lesser extend (see Sect. 5.4).

Note that if one changes the mean angle $\theta$ (the alignment of the $\mathrm{FP}$ with respect to the mirror) the asymmetries change differently because $r>0$ does no longer automatically lead to a blue-shift. Therefore there can also be asymmetries towards the red and the overall contrast will be diminished for all fiber sizes because the range of occurring $\theta$ increases. Using light distributions other than uniform distributions also changes the contrast and asymmetry of the FP signal because different areas of the fiber head (and therefore different rays crossing the FP) will have a larger weight in the total spectrum. 


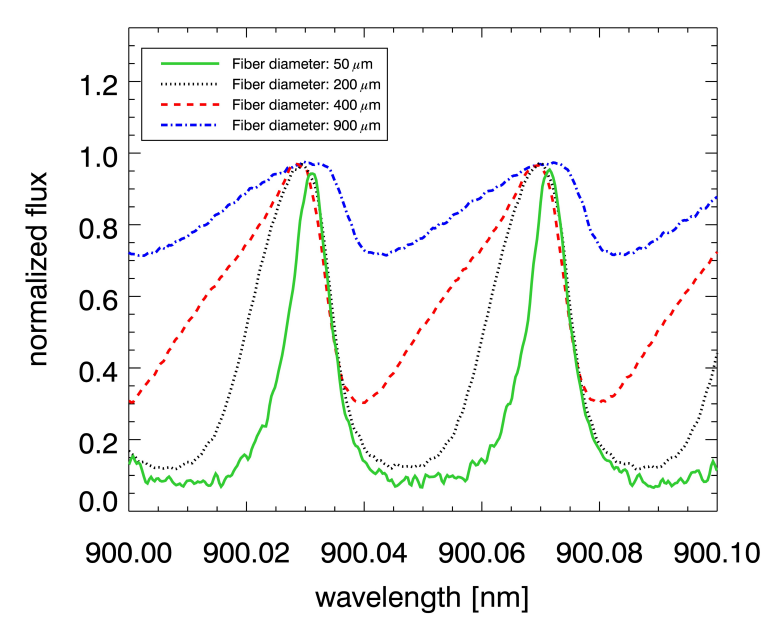

Figure 5.3: FTS measurements of a Fabry-Pérot using different fiber diameters $(R=1,000,000$ at $1000 \mathrm{~nm})$.

\subsection{Laboratory measurements}

Since astronomical spectrographs lack the resolution required to see the asymmetries shown in Sect. 5.2 one needs to use laboratory measurements to confirm these results. We measure the transmission spectrum of a FP using a Bruker Optics IFS 125 Fourier Transform Spectrometer (FTS) with a resolving power of $R=1,000,000$ at $1000 \mathrm{~nm}$. The interferogram is converted by the OPUS 7 software using a Mertz phase correction. As a white light source we use a halogen lamp which is coupled into a fiber (fiber A). To collimate the light coming from fiber $\mathrm{A}$ and send it through the FP we use a Thorlabs parabolic mirror.

Figure 5.3 shows the normalized measurements for different diameters of fiber A. These results bear close resemblance to our simulations, illustrated in Fig 5.2: The contrast decreases for larger fibers, approximately matching the values of the simulation. The same holds true for the asymmetry of the lines: while the $50 \mu \mathrm{m}$ fiber creates almost symmetrical peaks, the $900 \mu \mathrm{m}$ fiber looks heavily serrated, in fact the measurements show even larger asymmetries compared to the simulation. The peak positions differ for the majority of the fibers. However, contrary to the simulation the blue-shift does not clearly increase with fiber-size. Following the discussion in Sect. 5.2, we believe that this is probably caused by different mean angles $\theta$ during different measurements due to plugging and unplugging of the fibers. Additionally, our measurements were carried out with no particular temperature and pressure stabilization of the FP besides the room being temperature stabilized to $1 \mathrm{~K}$, which may also have influenced the peak positions through a change of the refractive index.

\subsection{Simulations of RV-shifts}

The simulated spectra in Fig. 5.2 are based on the assumption of a symmetrical light distribution at the exit of fiber A. This would require a perfect illumination of the entrance of this fiber (optimal launch conditions). Depending on the fiber used, the scrambling property of the fiber will level out deviations from that to some degree (Avila et al., 2006). However, this scrambling can be quite sensitive to movements of the fiber itself, making the light distribution at the exit of fiber A a time dependent function, even without taking into account severe changes of the initial launch conditions (e.g. changing the light bulb).

The scrambling factor describes how a fiber evens out an offset of the light input (in the form of a small laser dot) in terms of an offset of the barycenter at the exit. A very high scrambling factor would therefore always lead to a perfectly symmetrical light distribution with no offset of the barycenter. Unfortunately, a large scrambling factor does not automatically lead to small RV-shifts of the FP spectrum: in the most extreme case the light distribution at the exit of a fiber could be a ring with no flux in the center. The barycenter would still be at the midpoint of the fiber hence the fiber still has a very high scrambling factor. However, it is not only the barycenter shift that influences the FP peak positions, but also the light distribution pattern itself (more precisely the difference of the angle distribution of the rays crossing the FP), partic- 
ularly in the outer regions of the fiber. We have already shown that the larger area of the outer annuli translates to a higher weight of the spectral pattern created from these rays, which leads to a RV-shift with respect to a uniform fiber illumination (see Sect. 5.2). Thus, the scrambling factor only describes a connection between the barycenter of the light distribution on the entrance and the exit of the fiber, but it is not a useful quantity to describe aspects of the light distribution important for the RV-stability of a FP.

Although the scrambling factor is not a useful measure of the effective shift of the light distribution, it still can be used to get an idea of the magnitude of the problem. For example: depending on the optics used for coupling and the diameter of the fiber, scrambling factors can be as low as 25 but can also be higher than 500 (Avila and Singh, 2008). Shifting the light source by $50 \%$ of the fiber diameter would result in a barycentric shift on the fiber exit of $2 \%$ for a scrambling factor of 25 or $0.1 \%$ for a scrambling factor of 500 . While it is possible to reach even higher scrambling factors with techniques like fiber squeezing or optical scrambling we do not consider them here because these techniques are usually not used in combination with FPs.

\subsubsection{Light distribution}

As an approximation of changes in the fiber launch conditions, we investigate the consequences of a barycenter shift considering different light distributions on the fiber exit of fiber A. For this we assume the light pattern to be circular and uniformly distributed, but only reaching out to $90 \%$ of the fiber radius, effectively shrinking the fiber. Additionally, we introduce an offset of this circular distribution to simulate the effect of imperfect scrambling. We use offsets of $0.1,1,2,3,4$ and $5 \%$ of the fiber diameter for typical fiber diameters of 400, 200, 100, 50 and $10 \mu \mathrm{m}$. The perimeters of these distributions are shown in Fig. 5.4. In order to calculate the to-

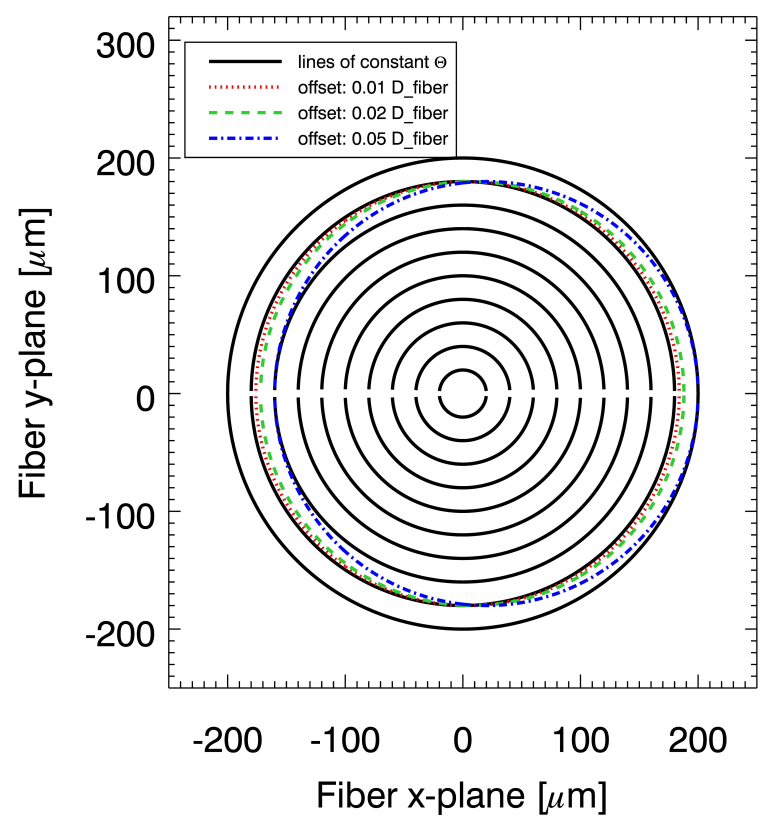

Figure 5.4: Contour of the circular light distribution of a $400 \mu \mathrm{m}$ fiber with offsets of 4,8 and $20 \mu \mathrm{m}$ (1,2 and $5 \%$ of the fiber diameter)

tal spectrum of the FP one needs to convert the light distribution on the fiber exit into a weighting function for all occurring angles $\theta_{d e v}$ (and therefore as a function of distance from the fiber midpoint, see Eq. 5.1), as shown in Sec. 5.2. For the symmetrical case without any offset, this weighting function is proportional to the distance from the fiber midpoint (see Eq. 5.2). Calculating the weighting function with the introduced offset is done in two steps. The inner part of the fiber is still fully illuminated and since we use a uniform distribution for the light intensity this looks exactly the same as without the offset. For the outer regions, where only parts of each annulus is illuminated one can compute the area as a function of distance from the midpoint by calculating the circle-circle intersection ${ }^{2}$ between

2 Weisstein, Eric W. "Circle-Circle Intersection." From MathWorld-A Wolfram Web Resource. http://mathworld.wolfram.com/ Circle-CircleIntersection.html 


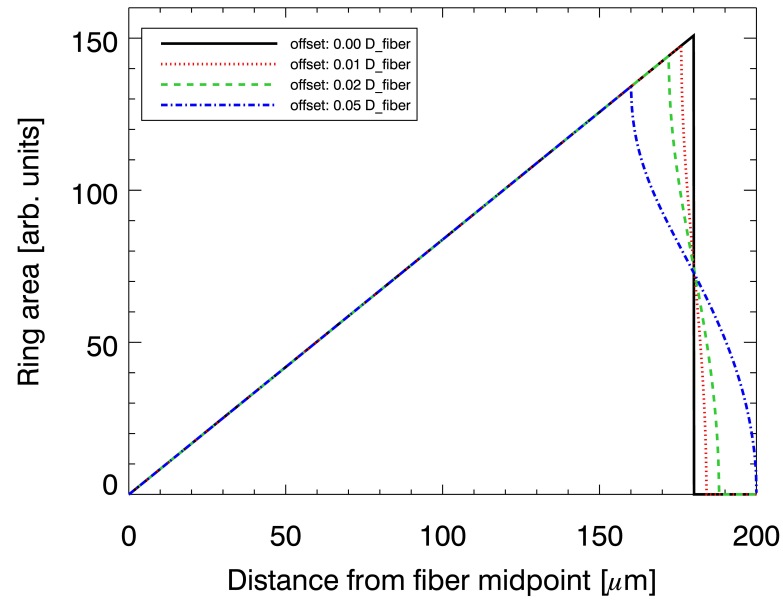

Figure 5.5: Illuminated area as a function of distance $D$ from the fiber midpoint (see Eq. 5.1) for different offsets of the light distribution on the exit of a $400 \mu \mathrm{m}$ fiber (see Fig. 5.4). This weighting function is then used to add up the different spectra for each annulus in order to get the total spectrum (Fig. 5.6).

the offset light distribution and the fiber head. This leads to an arccos shaped function for the outer parts of the fiber head (Fig. 5.5). To get the final transmission spectrum of the FP all spectra from different angles have to be added up according to this weighting function (see Sec. 5.2).

Assuming a uniform light distribution is a compromise between realistic light distributions (like Gaussian distributions or top-hat profiles) and a high resolution of the weighting function. In order to simulate RV-shifts of the final spectrum of the order of a few $\mathrm{cm} \mathrm{s}^{-1}$ the weighting function should ideally be calculated analytically. Doing so with the circle-circle intersection method requires circular symmetric light distributions, hence the introduction of an offset prevents the usage of Gaussian distributions, since those are no longer symmetric with respect to the fiber.

In principle one could monitor the actual light distribution at the exit of Fiber A (e.g.

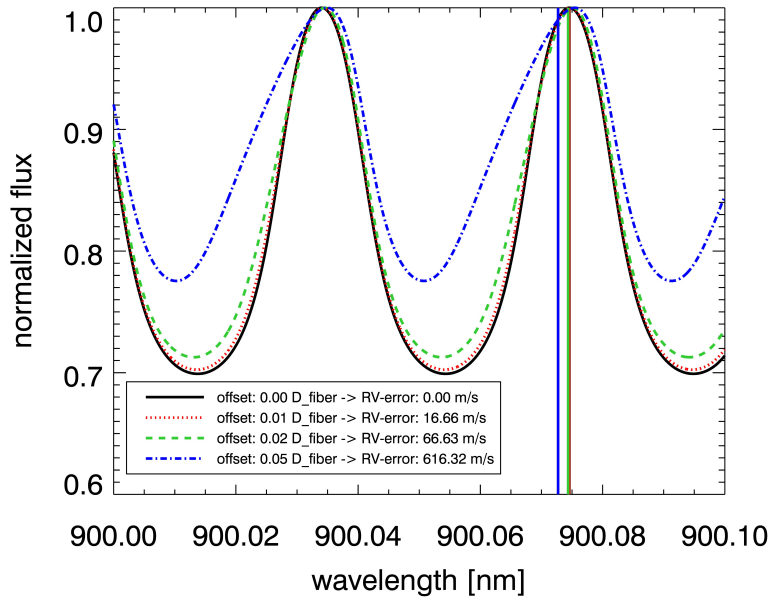

Figure 5.6: Simulated FP transmission spectra for a $400 \mu \mathrm{m}$ fiber and an offset of 1,2 and $5 \%$ at the fiber exit and the resulting RV-error. The barycenter for each offset is marked by a vertical line.

by using a beamsplitter in front of the FP) of a given setup and forward model RV-shifts due to changing light distributions. Although this approach would be more realistic it would also need much more computational power in order to achieve the same RV-precision as our analytical model. Here, we are more interested in the general behavior of these systems rather than in the performance of a specific setup, and we refrain from designing more elaborated setups for the purpose of this paper.

\subsubsection{Impact on RV-measurements}

Following the same argument as in Sect. 5.2, the introduction of an offset of the light distribution leads to asymmetric peaks and reduced contrast. Both effects scale proportional to the offset and like in Sect. 5.2, the asymmetry effectively introduces a blue-shift of the peak positions. Figure 5.6 shows the FP spectra for a $400 \mu \mathrm{m}$ fiber and offsets of 1,2 and $5 \%$. Astrophysical spectrographs will most likely not fully resolve the FP signal (see Fig. 4.2). Instead, one can measure the barycenter of each 


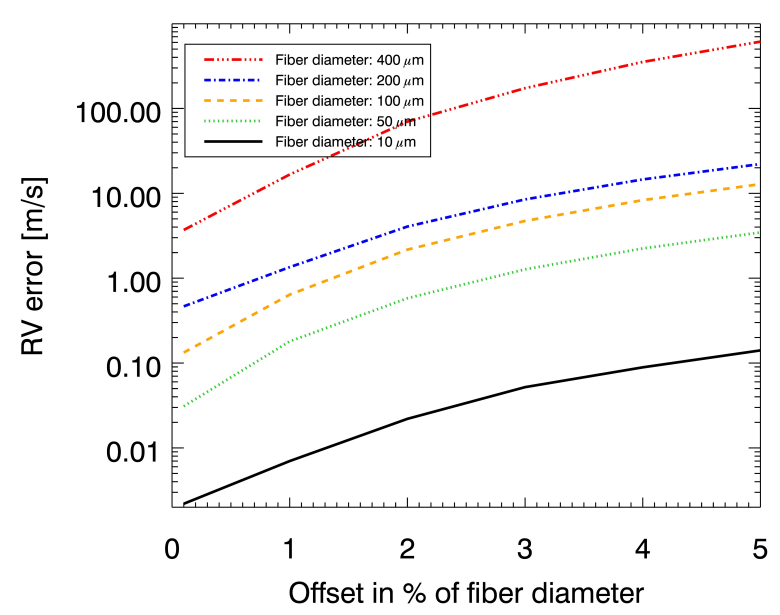

Figure 5.7: Resulting RV-errors due to an offset of the light distribution on the fiber exit.

peak, which can then be used to determine the drift of the spectrograph. In order to simulate this procedure we calculated the barycenter of each peak numerically (from one minimum to the next one). The shift of this barycenter due to the offset of the light distribution can then be turned into a RV-error using Eq. 4.4.

Figure 5.7 shows the result for a number of commonly used fiber diameters with offsets of the light distribution ranging between $0.1 \%$ and $5 \%$. The resulting RV-error depends massively on the fiber diameter. Fiber diameters of $400 \mu \mathrm{m}$ (top line, dashed-tripledotted) with offsets of the light distribution as small as $0.1 \%$ lead to RV-errors of more than $3 \mathrm{~m} \mathrm{~s}^{-1}$. For a $10 \mu \mathrm{m}$ fiber (bottom line, solid), even offsets as large as $5 \%$ result in RV-errors of only $15 \mathrm{~cm} \mathrm{~s}^{-1}$. In order to reach a $1 \mathrm{~m} \mathrm{~s}^{-1}$ RV precision using a $400 \mu \mathrm{m}$ fiber one has to ensure an offset of much less than $0.1 \%$, while for fibers smaller than $200 \mu \mathrm{m}$ (dashed-dotted line) offsets of $1 \%$ are sufficient. Naturally, reaching a RV-stability better than $10 \mathrm{~cm} \mathrm{~s}^{-1}$ requires even more stable conditions of the light distribution: for a $10 \mu \mathrm{m}$ fiber even an offset as large as $4 \%$ is good enough. Fiber diameter of $100 \mu \mathrm{m}$ (dashed line) already require offsets smaller than $0.1 \%$ to reach a
RV-stability of the same order. For fibers larger than that, the necessary stability of the light distribution become extremely challenging, with offset limits of the order of $0.01 \%$. In terms of barycentric offset this would require scrambling factors of better than 5000 (however, see the discussion about the scrambling factor in Sect. 5.4).

Changes of the light distribution may occur on different timescales: The launch conditions can be stable on timescales of weeks or months but may change abruptly (e.g. due to bulb replacements). The filament of light bulbs can change due to heating and introduce a shift of the light distribution on the fiber head (timescales of days). Both, moving the fiber (e.g. bending it just a bit) and vibrations (e.g. of vacuum pumps) will change the scrambling factor and therefore the barycentric shift on the exit of the fiber on timescales depending on the disturbing cause.

\subsection{Summary}

We investigated the impact of fiber-related effects on the RV-performance of Fabry-Pérot Etalons. While the impact of pressure and temperature variations has been discussed before, we show how the fiber diameter and illumination influence the resulting RV-stability.

We investigated how different fiber-sizes influence the spectrum of a FP. We find excellent agreement between our simulations and very high resolution laboratory measurements, showing that increasing the fiber diameter leads to a diminished contrast of the FP signal and shifted peaks.

Our most important result is that changes of the light distribution on the fiber head in front of the FP will lead to significant RVshifts of the final FP spectrum. We computed a number of different light distributions in order to simulate incomplete scrambling of the fibers, shifting the barycenter on the fiber head. These changes of the light distribution lead to different distributions of rays crossing the FP which results in blue-shifted peaks 
and asymmetric features in the total spectrum. These effects can range between only a few $\mathrm{cm} \mathrm{s}^{-1}$ for very small fibers $(10 \mu \mathrm{m}$ diameter) and more than $10 \mathrm{~m} \mathrm{~s}^{-1}$ for fiber diameters of $400 \mu \mathrm{m}$.

Our results indicate that changes of the light distribution are heavily influencing the performance of a FP as a tool for wavelength calibration. Since these changes scale with the fiber diameter it is crucial to choose sufficiently small fibers with respect to the required RV-stability. Alternatively, the stability of the light distribution has to be measured directly in order to compensate for its fluctuations, e.g. as a part of the data reduction. In order to achieve a $1 \mathrm{~m} \mathrm{~s}^{-1} \mathrm{RV}$ stability fibers smaller than $200 \mu \mathrm{m}$ should be used while $10 \mathrm{~cm} \mathrm{~s}^{-1}$ requires fibers smaller than $50 \mu \mathrm{m}$ or puts extreme demands on the stability of the fiber illumination. 


\section{Chapter 6}

\section{Fabry-Pérot Etalon: Final setup for CARMENES}

This Chapter describes the final setup of the FP units for CARMENES, how they works and which components are used. All simulations and measurements are focused on the visible part of the CARMENES wavelength range. However, the near-infrared FP unit will be built in the same way but with a different FP-coating, optimized for the NIR.

\subsection{Vacuum tank}

While useful for the firsts test, the cage system shown in Sec. 4.2.2 is not suitable for the final setup inside a vacuum tank. After some tests it became clear that the cage itself does not provide the stability needed in terms of tension-free alignment. Therefore, a new stage to be mounted inside a vacuum tank has been designed.

The tank consists of two DN $200 \mathrm{CF}$ flanges on the bottom and top side and a custom made, double layered cylindrical body (see Fig. 6.1). Between the two layers a coolant (silicon oil) provides the necessary temperature stabilization (see Sec. 6.6). Both DN $200 \mathrm{CF}$ flanges have been equipped with three DN $40 \mathrm{CF}$ flanges each to provide enough connection possibilities. They are used as follows:

- a right-angle DN $40 \mathrm{CF}$ to $40 \mathrm{KF}$ adapter to connect the hose leading to the vacuum pump

- a venting valve to allow for venting of the tank without using the venting function of the pump

- two custom made DN 40 CF vacuum fiberfeedthroughs, one for the input and one for the output fiber

- a Pirani/Cold Cathode gauge PKR 251 by Pfeiffer Vacuum to measure the pressure inside the tank

- an electrical feedthrough for four PT100 temperature sensors

For testing the FP unit in the lab we use a Pfeiffer HighCube with a HiPace 300 turbopump and a Duo 2.5 backing pump. This device is much more powerful than what is actually needed in order to reach a pressure of $4 \cdot 10^{-3}$ mbar (see Sec. 4.1.3). The final pumps for usage at at Calar Alto have yet to be chosen, but they will most likely be backing pumps only, since high end backing pumps can reach the required pressure and are much more cost- and energy-efficient than a backing pump/turbopump-combination. In order to be able to vent the tank without using the pump we also installed a venting valve. This could become useful in case there will be only one pump for both, the NIR and the visual FP unit and only one of the tanks has to be vented.

The fiber-feedthroughs are self made by Julian Stürmer in Heidelberg (who is working on the science fibers for CARMENES) and 

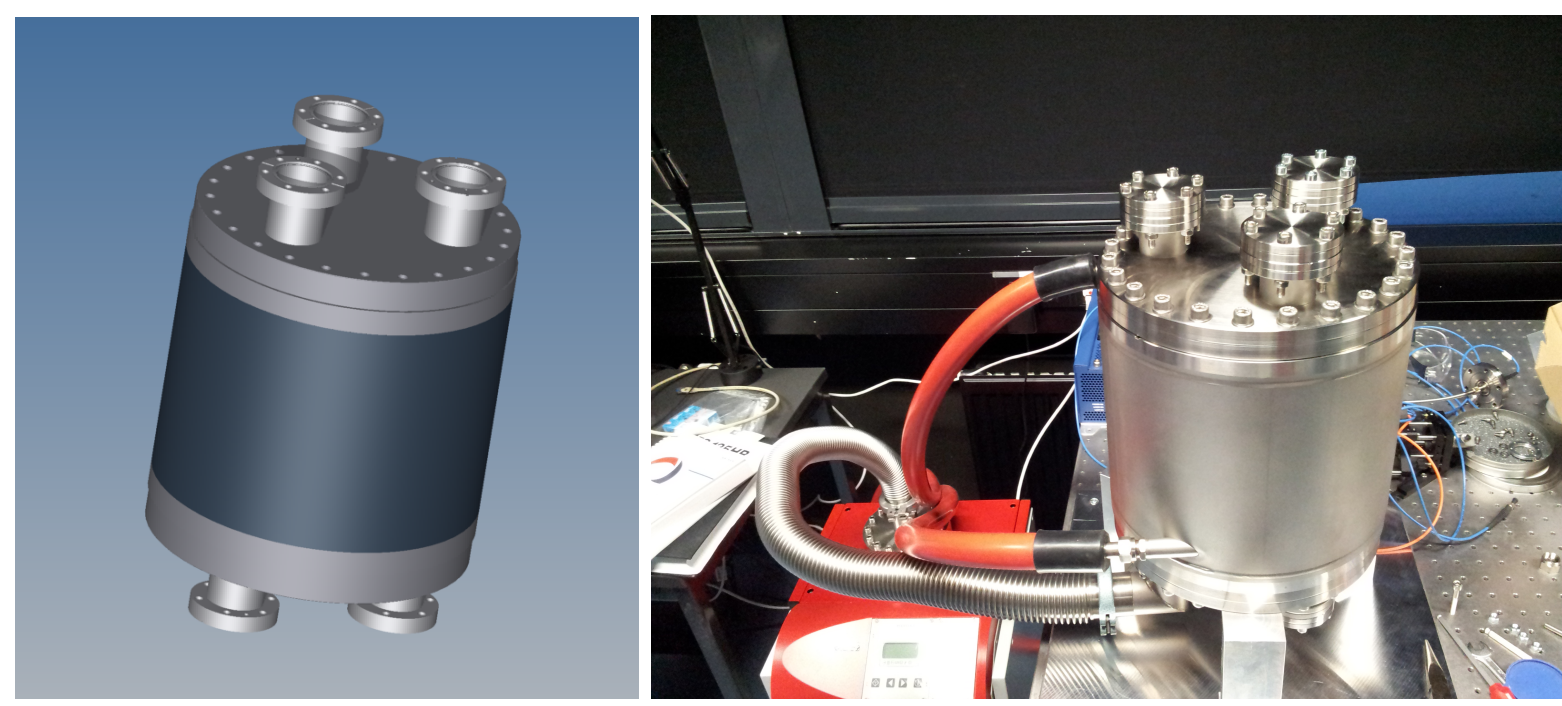

Figure 6.1: Left: CAD drawing of the tank. Right: Tank connected with vacuum pump (silver hose) and thermopump (red hoses).
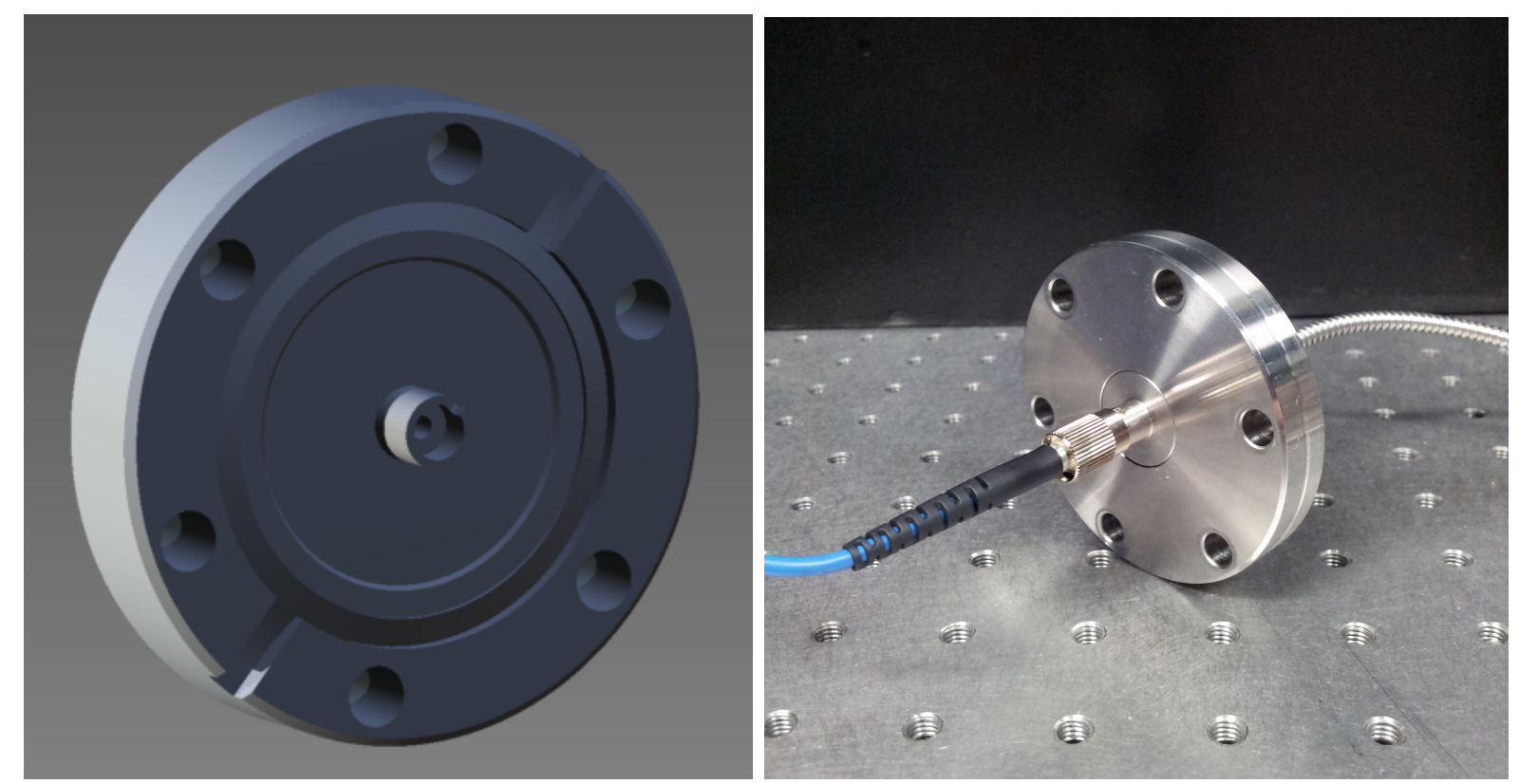

Figure 6.2: Left: CAD drawing of the DN $40 \mathrm{CF}$ fiber feedthrough flange. Right: The assembled vacuum feedthrough with fiber protection tubes on both sides. 
myself. Figure 6.2 shows a CAD drawing of the flange without the fiber and a picture of the finalized feedthrough. While feedthroughs with fiber connectors can be bought directly from different companies, these feedthroughs always come with the drawback of having a small piece of fiber inside the flange. This not only means that there will be a light loss due to the two connectors (one at each side) but also the fiber diameter and fiber material inside the flange can't be chosen according to our requirements. Therefore the self made solution with one fiber crossing the feedthrough and being glued into the flange has been preferred. FC connectors are used to provide the mechanical stability for the fiber at the flange.

The Pirani/Cold Cathode gauge PKR 251 by Pfeiffer Vacuum has (according to Pfeiffer) an accuracy of $30 \%$ in the relevant pressure region around $4 \cdot 10^{-3}$ mbar. For our lab experiments this is sufficient since we can easily evacuate down to $1 \cdot 10^{-3}$ mbar using the Turbopump and therefore measure pressure fluctuations way below $4 \cdot 10^{-3} \mathrm{mbar}$, which would be the requirement for a $10 \mathrm{~cm} \mathrm{~s}^{-1} \mathrm{RV}$ precision (see Sec. 4.1.3). However, it depends on the final choice of pump for CARMENES whether or not this gauge is precise enough for usage at Calar Alto.

The temperature of the coolant will be controlled by the thermopump (see Sec. 6.6). Additionally, there will be temperature sensors mounted on the inner wall of the vacuum tank and on the optical bench close to the FP. For this PT100 sensors in combination with a LakeShore Model 224 temperature monitor will be used. This setup can measure temperature deviations of about $1 \mathrm{mK}$ (compared to the $15 \mathrm{mK}$ requirement for the temperature stability of the air inside the FPs and the $10 \mathrm{mK}$ temperature stability provided by the thermopump (see Sec. 6.6).

\subsection{Optical bench}

Figure 6.3 shows a CAD drawing of the bench with the layout of the off-axis parabolic mir- rors and the FP, Fig. 6.4 shows the unit in the lab. The stage has three legs to be mounted directly on the bottom (or hanging from the top) of the vacuum tank. The matching threads for the mirrors are drilled into slide-in modules which are held in place inside the blocks (orange) using four screws. The FP is also hold in place in its block by screws.

In order to avoid air pockets inside the threading holes, hollow screws are used. Both, the stage and the blocks holding the optics are made of Aluminum and the stage is cut out of a single block in order to avoid tensions which could potentially change the alignment of the optics. The block holding the FP has multiple vent holes to ensure air exchange between the inside of the FP and the tank.

\subsection{Parabolic mirrors}

In order to avoid chromatic aberration and increase the overall efficiency of the system, parabolic mirrors are used instead of lenses. The mirrors (RC08SMA-P01 from Thorlabs) are silver coated to provide high efficiency over the whole CARMENES wavelength range (550 $1700 \mathrm{~nm}$ ). They have SMA connectors to allow the fibers to be plugged in directly without any further alignment needed. The matching threads for the mirrors are drilled into slide-in modules which are held in place inside the blocks using four screws (see Fig. 6.3, colored in blue). 


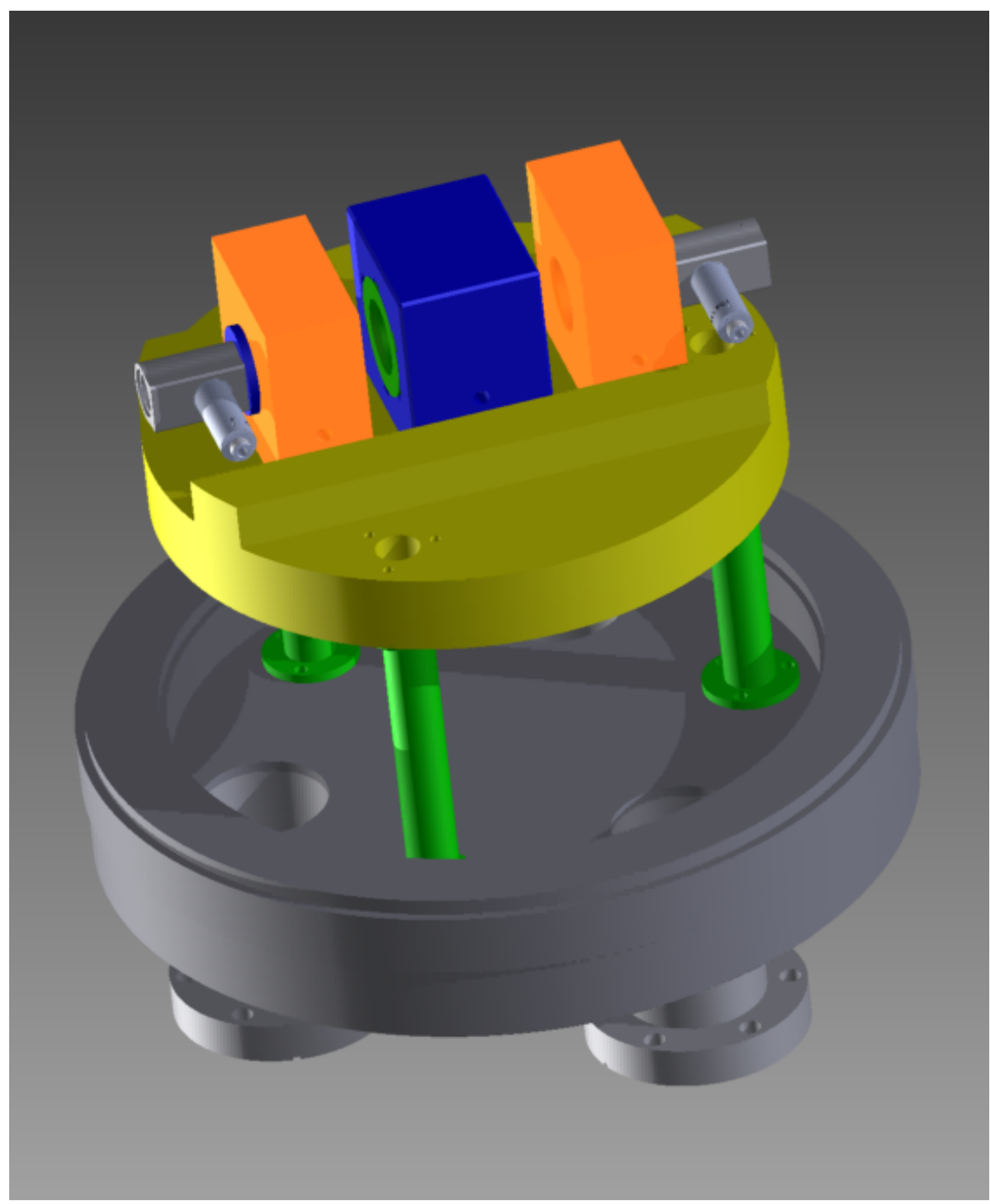

Figure 6.3: CAD-drawing of the optical bench. The off-axis parabolic mirrors are directly screwed into blocks of Aluminum (shown in orange), the FP (green) is mounted inside the middle block (blue) and held in place by one screw. 


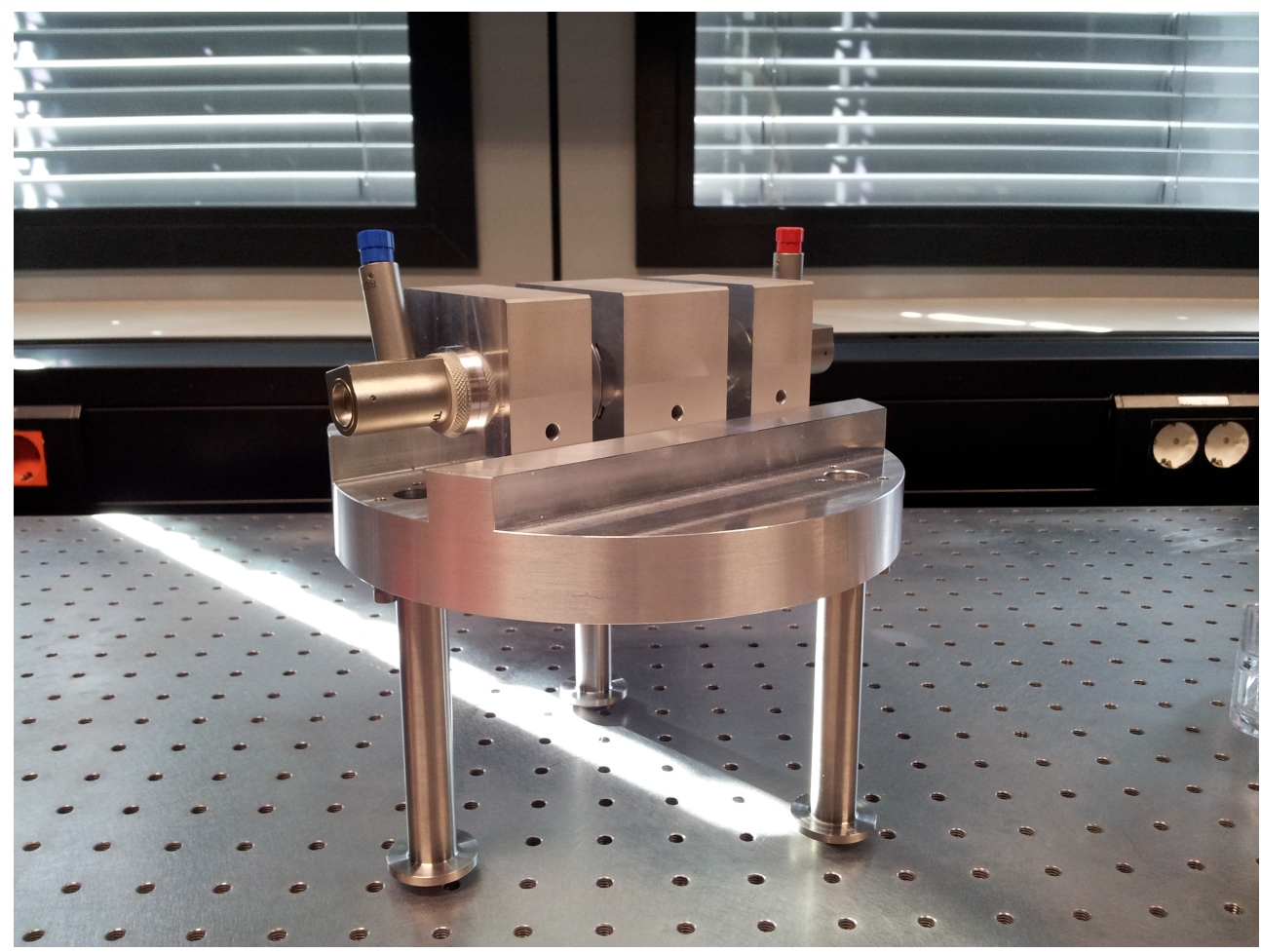

Figure 6.4: The optical bench standing with both its off-axis parabolic mirrors attached and the FP fixed inside the middle block.

\subsection{Fabry-Pérot}

The FPs are manufactured by SLS optics Ltd.. The blue FP is optimized for the wavelength range between 600 and $1000 \mathrm{~nm}$. Its Schott Zerodur spacer holds the optical surfaces at a distance of $9.990 \pm 0.001 \mathrm{~mm}$ (the error is due to the manufacturing process) and is built into a $38 \mathrm{~mm}$ diameter aluminum cell with vent holes. The optical surfaces have a soft coating and are wedged by 30 arcmin. The effective finesse is $\mathrm{Fe} \approx 8$ (it varies as a function of wavelength between 7.63 and 8.20), resulting in a free spectral range (the peak to peak distance) as measured by SLS optics Ltd. between $0.018 \mathrm{~nm}$ at $600 \mathrm{~nm}, 0.032 \mathrm{~nm}$ at $800 \mathrm{~nm}$ and $0.05 \mathrm{~nm}$ at $1000 \mathrm{~nm}$.

The red FP is optimized for the wavelength range between 900 and $1300 \mathrm{~nm}$ with Zerodur spacers of $12.334 \pm 0.002 \mathrm{~mm}$. As for the blue $\mathrm{FP}$ its finesse is $\mathrm{Fe} \approx 8$ (varying between 7.94 and 8.14) and uses a wedged soft coating. The resulting free spectral range (measured

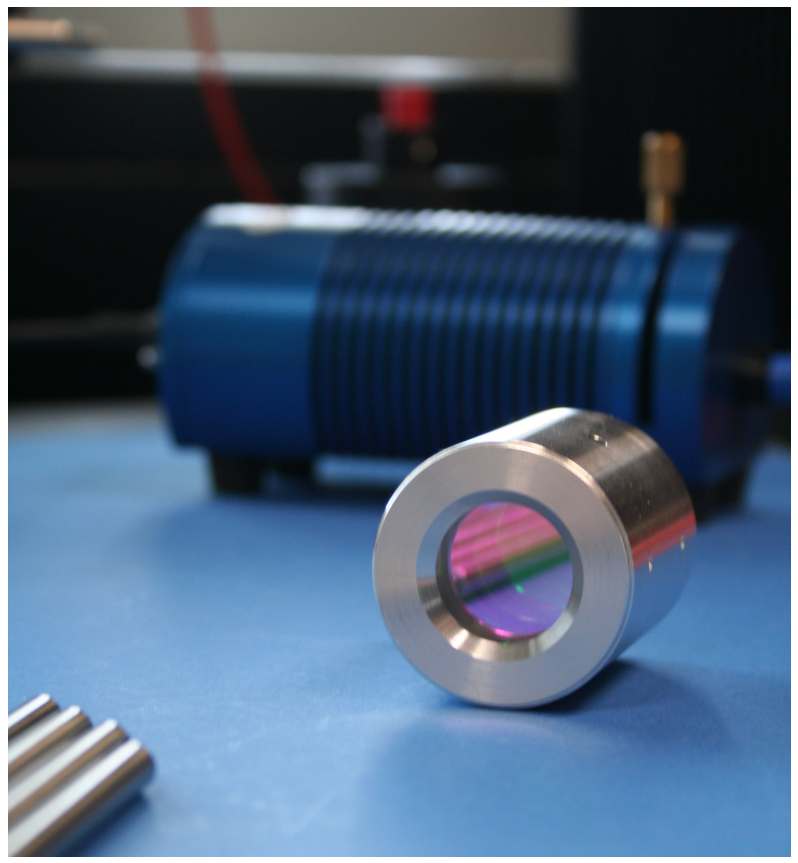

Figure 6.5: Photo of the blue FabryPérot. 
by SLS optics Ltd.) is $0.032 \mathrm{~nm}$ at $900 \mathrm{~nm}$, $0.049 \mathrm{~nm}$ at $1100 \mathrm{~nm}$ and $0.068 \mathrm{~nm}$ at $1300 \mathrm{~nm}$.

Figure 6.5 shows a photograph of the blue $\mathrm{FP}$ in the optical lab. Once inside its aluminum block (see Fig. 6.3) it is held in place by a screw.

\subsection{Fibers and light source}

As a result of the detailed investigations with regard to the diameter of the fiber feeding the light into the FP device, shown in Sec. 5, fiber A will either be a $10 \mu \mathrm{m}$ multimode fiber (Thorlabs HPSC10) with a NA of 0.1 or a $50 \mu \mathrm{m}$ multimode fiber with a NA of 0.22 (Thorlabs FG050LGA). For the outgoing fiber B, leading to the spectrograph (or, in the case of CARMENES, to the calibration unit), a $200 \mu \mathrm{m}$ fiber with a NA of 0.22 (Thorlabs AFS200/220Y) is used in order to avoid any further light losses while coupling the FPs transmission back into the fiber. Using the same fiber size for fiber B as for fiber A would require an extremely precise alignment, e.g. using a kinematic mount. This would introduce movable parts into the FP unit, which should be avoided if possible. Also, using a smaller fiber $\mathrm{B}$ does not improve the performance of the FP unit and therefore the $200 \mu \mathrm{m}$ fiber has been selected.

Using a fiber as small as $10 \mu \mathrm{m}$ as fiber A ensures that changes of the launch condition and fiber scrambling effects can't introduce RV-errors larger than a few $\mathrm{cm} \mathrm{s}^{-1}$ even in a worst case scenario. However, it also reduces the amount of flux reaching the spectrograph, hence we also consider a $50 \mu \mathrm{m}$ fiber (see Sec. 6.7). Most tests up to this point have been done using a Fourier Transform Spectrometer (Bruker IFS 125). Its efficiency is orders of magnitudes below that of astrophysical spectrographs like CARMENES. Therefore a laser-driven light source (LDLS) by Energe$\mathrm{tiq}^{1}$ is used to ensure a sufficient level of flux for the FTS measurements. Inside the LDLS

\footnotetext{
1 http://www.energetiq. com/html/eq99FC.html
}

a NIR-laser is focused onto a neon plasma (see Fig. 6.6). The plasma is heated up in a very small area $(\mathrm{d} \sim 100 \mu \mathrm{m})$ and therefore produces a very bright light spot with an extremely high flux density. Using such a light source makes it possible to measure the spectrum of a FP using a fiber as small as $10 \mu \mathrm{m}$ even with a FTS.

For CARMENES Halogen lamps will provide sufficient flux because of the much higher sensitivity of the spectrograph compared to the FTS. Currently a LOT Halogen lamp housing (LSB110/5) is used with a $10 \mathrm{~W}$ bulb. The housing is passively cooled without any moving parts to avoid vibrations and to provide the most stable lamp output. The light from the bulb is first collimated by a lens integrated in the housing and than focused on the fiber head using the same off-axis parabolic mirror as inside the vacuum tank.

\subsection{Cooling concept}

The target temperature for the FP units will be $15^{\circ} \mathrm{C}$, which is the temperature where the index of expansion of the Zerodur spacers is at its minimum. Providing a temperature stability of $15 \mathrm{mK}$ inside the tank is quite challenging. Although one could use an electrical heating module to keep the temperature of the FP itself stable, there are a number of issues with this idea: The main problem is, that the relatively small mass of the FP itself is not providing enough inertia to suppress fast temperature changes. Additionally, it is not only the temperature of the FP but also the temperature of the air inside that has to be stabilized. Finally, electrical heating would almost certainly lead to temperature gradients within the tank, making it very hard to precisely regulate the temperature of the air inside the FP. Therefore, a different cooling/heating strategy is used:

The vacuum tank is built with two separate layers, leaving a gap in between (see Fig. 6.7, left). A coolant (silicon oil) is pumped into this intermediate space on the bottom 


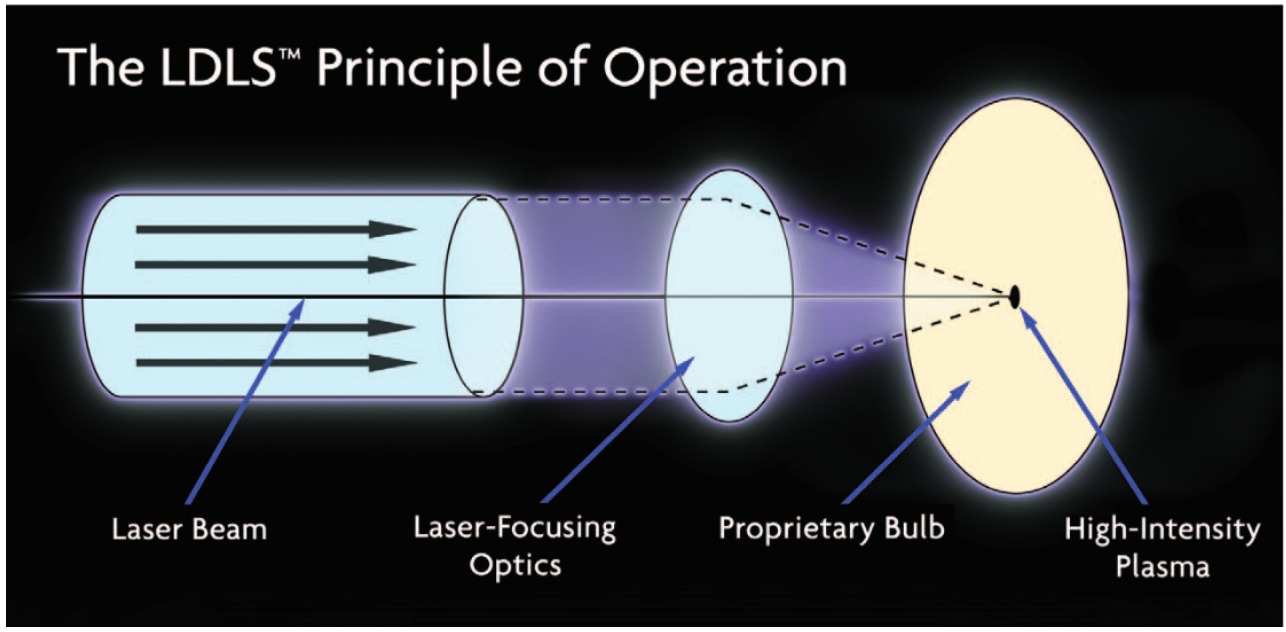

Figure 6.6: The LDLS Principle of operation (source: http://www.energetiq.com/DataSheets/EQ99FC_Data_Sheet.pdf).
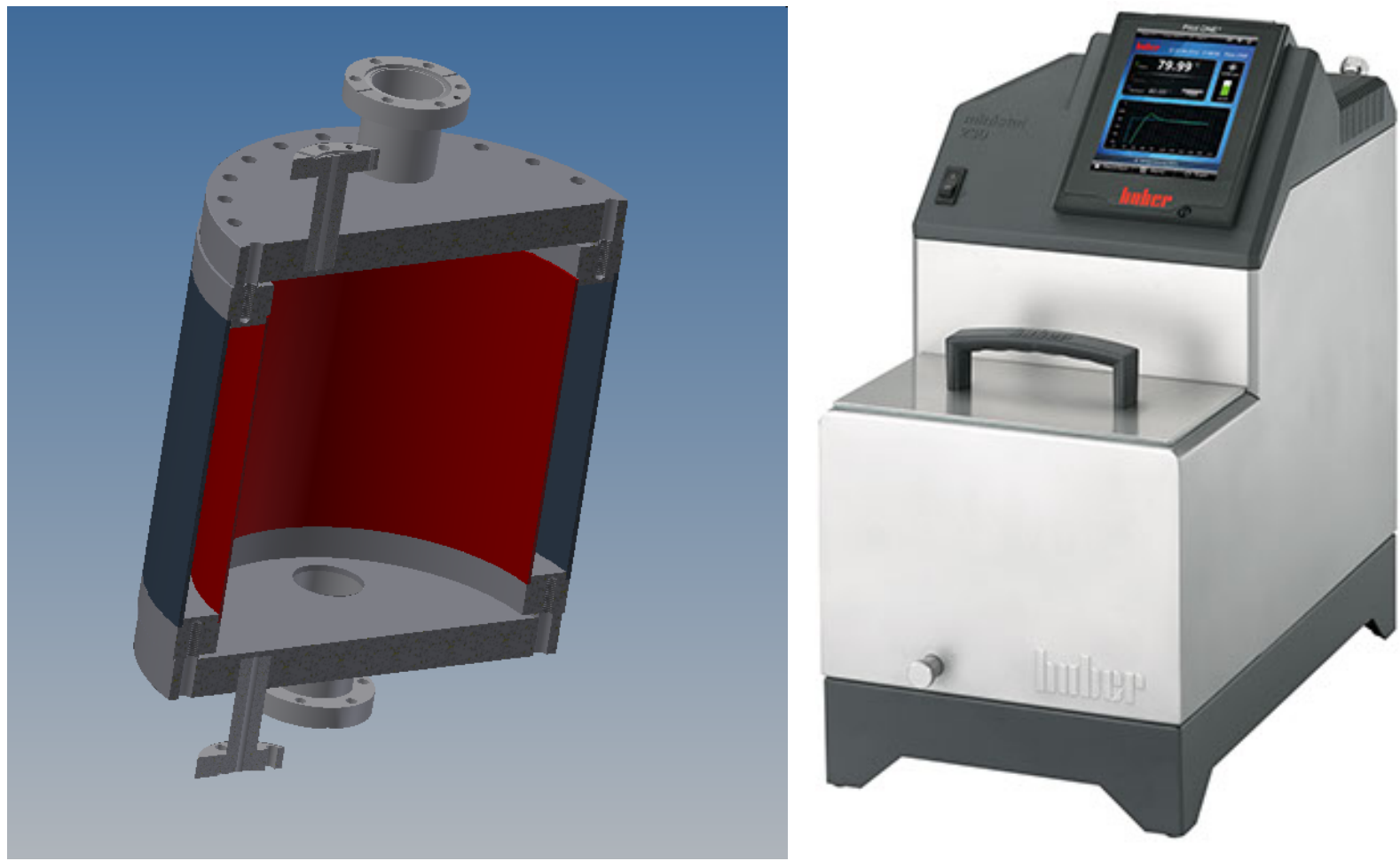

Figure 6.7: Left: Section view of a CAD drawing of the vacuum tank. The $46 \mathrm{~mm}$ wide gap between the outer (blue) and inner layer (red) contains the silicon oil which circulates upwards (inlet and outlet hose attachments not shown). Right: The Huber Ministat 230 cooling circulator (source: http://www.huberonline.com/de/product_datasheet. aspx?no=2015.0012.01). 


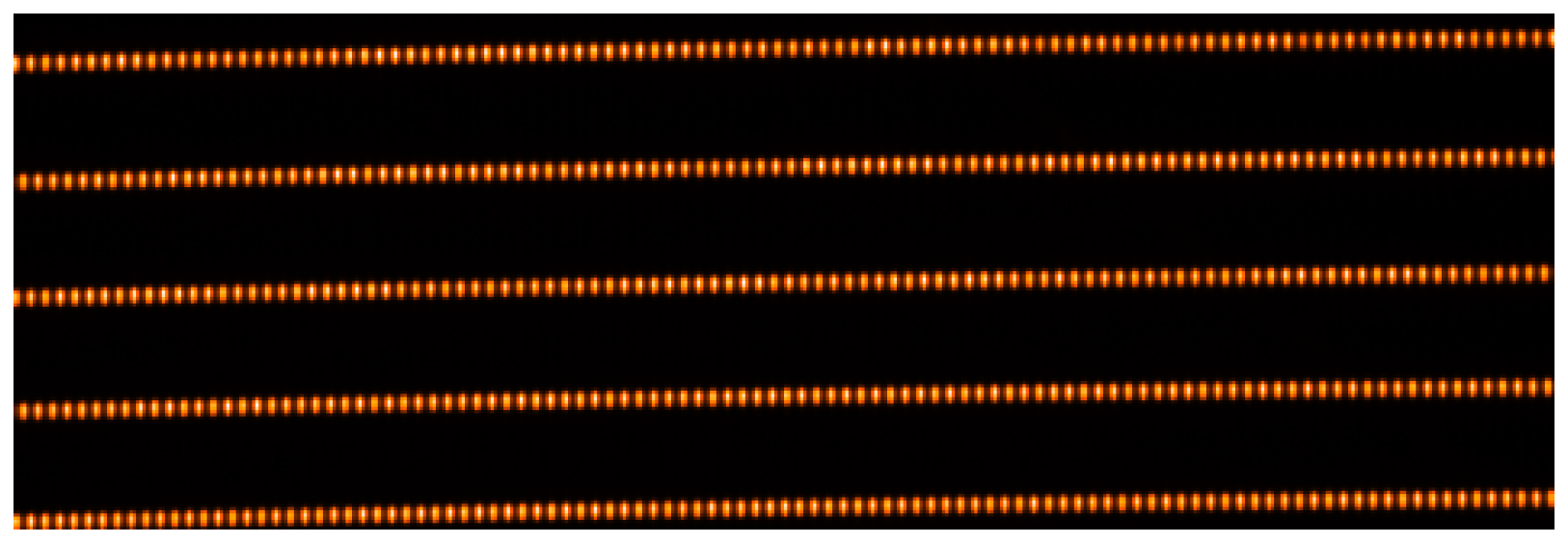

Figure 6.8: Part of the CCD raw image of the visual FP, using a Halogen lamp and a $50 \mu \mathrm{m}$ fiber (taken at Tautenburg with $\mathrm{R}=67,000$ and the IR setup of the spectrograph).

side of the tank and exits the tank on the upper side. Due to the cylindrical shape of the tank, the coolant is circulating upwards, effectively adjusting the temperature of the whole vacuum tank to the temperature of the silicon oil.

The silicon oil is temperated by a cooling circulator, a Huber Ministat 230 (see Fig. 6.7, right) which is connected to the vacuum tank using insulated, reinforced hoses. The Ministat has a heating capacity of $2 \mathrm{~kW}$ and a cooling capacity (depending on the target temperature) of about $0.4 \mathrm{~kW}$. It has two separate pumps, a pressure pump with a maximum flow rate of $27 \mathrm{lpm}$ and a suction pump with a maximum flow rate of $20 \mathrm{lpm}$. It uses a 5-point calibration with multiple PT100 sensors to reach the target temperature, either by only checking the back-flow temperature or using additional input from the process temperature. With an additional upgrade to the 'E-grade Exclusive' controller, this device is able to keep the coolant at the required temperature $\pm 10 \mathrm{mK}$ within a temperature range between -40 and $200^{\circ} \mathrm{C}$. By adjusting the temperature of the coolant, the temperature of the vacuum tank will be kept stable at the target temperature at all times. Similarly, the air inside will be stabilized to the same degree. Since the optical bench is physically connected to the tank it will also adapt its tem- perature to that value. Additionally, the inside of the tank and the optical bench are connected with temperature bridges in the form of copper bands to improve the conductibility of temperature between the stage and the tank. Due to the high mass of the tank this setup will be insensitive to temperature changes of the room on small timescales (somebody enters the room), while changes on longer timescales (summer/winter) will be compensated by the Ministat cooling circulator.

\subsection{First light}

In order to carry out the most meaningful test in preparation for CARMENES, the FP unit has been tested at the Thüringer Landessternwarte Tautenburg, where both calibration units for CARMENES are built. The calibration units host a number of emission lamps (ThNe for the calibration unit of the visual spectrograph and UNe for the NIR spectrograph), a flat field lamp and an input for the FP. One can select which lamp is going into the calibration fiber leading to the spectrograph by rotating a mirror while the FP is coupled in by using a beamsplitter. We simulated the impact of the front end of the $3.5 \mathrm{~m}$ telescope on Calar Alto by including a FC-FC adapter. Using this adapter, the fiber diameter of the fiber leading from the calibra- 


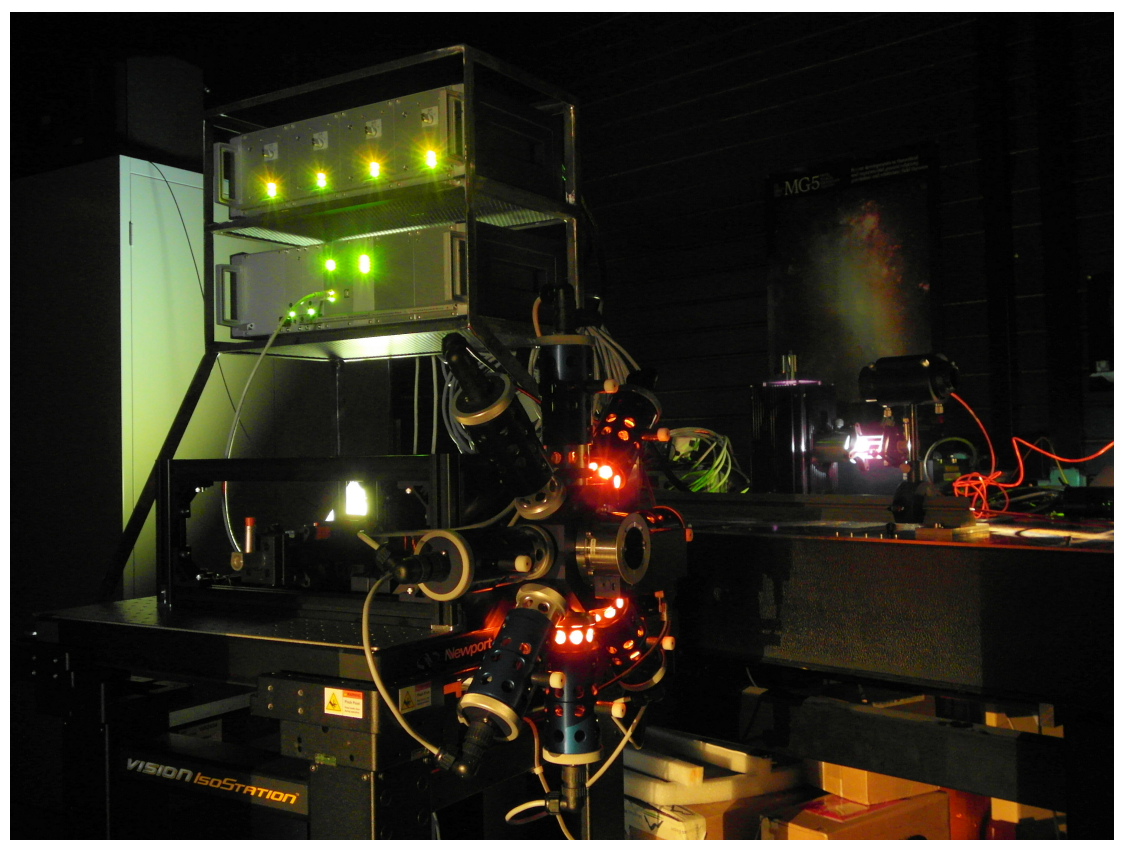

Figure 6.9: First light of the calibration unit in combination with the FP unit. The 'wheel' containing the calibration lamps is in the center, the calibration unit with its electronics is on the left and in the right background the Halogen lamp of the FP unit and one of the fibers can be seen.

tion unit to the Tautenburg spectrograph is reduced by the same amount as the front end will do on Calar Alto.

The spectrograph in Tautenburg is not stabilized, its resolution is slightly lower than the resolution of CARMENES $(\mathrm{R}=67,000 \mathrm{vs}$ $\mathrm{R}=82,000)$ and its efficiency may very well be up to 2 times lower than for CARMENES. However, it provides a good enough platform for testing exposure times and count-rates for different FP-setups, something that cannot be done at the FTS in our laboratory due to the different functional principle of the FTS compared to an echelle spectrograph. Figure 6.9 shows the setup in the Tautenburg laboratory, including the calibration unit.

FP Spectra with different exposure times (between one and 60 seconds) with different light-sources (two different Halogen lamps and the LDLS) and different fiber diameters (10 and $50 \mu \mathrm{m}$ ) were taken to be analyzed with respect to the resulting signal to noise ratios. The ThNe and UNe lamps of the calibration unit have also been measured for comparison. Unfortunately there is currently no pipeline available to reduce the data due to recently implemented optics in the Tautenburg spectrograph. However, first results from the raw frames (see Fig. 6.8) indicate that using a Halogen lamp and a $50 \mu \mathrm{m}$ fiber provides FP peaks with about the same intensity as the brightest Th lines for the same exposure time. This would be ideal for the proposed calibration strategy of CARMENES (see Sec. 3.3).

As expected from geometrical considerations (the flux $F$ scales with the fiber diameter $d$ as $F \propto d^{2}$ ) using a $10 \mu \mathrm{m}$ fiber produces about 25-30 times less flux. The LDLS provides about 30 times more light than the Halogen lamps, therefore using a LDLS would allow for using $10 \mu \mathrm{m}$ fibers with comparable results to the Halogen lamp with a $50 \mu \mathrm{m}$ fiber with respect to the peak intensity. 
38

CHAPTER 6. FABRY-PÉROT ETALON: FINAL SETUP FOR CARMENES 


\section{Chapter 7}

\section{XSHOOTER}

\subsection{Introduction \& connection to CARMENES}

The XSHOOTER instrument (Vernet et al., 2011) at the VLT is one of the few spectrographs covering the whole wavelength range of CARMENES. It is currently mounted at the UT2 Cassegrain focus and consists of three spectroscopic arms, each with different optics and detectors:

- UVB, optimized for $300-559.5 \mathrm{~nm}$

- VIS, optimized for 559.5-1024 nm

- NIR, optimized for 1024-2480 nm

Figure 7.1 shows a schematic overview of the XSHOOTER instrument. Like in CARMENES, each arm of the instrument is an independent, cross dispersed echelle spectrograph. Both, CARMENES and XSHOOTER allow for simultaneous observation of a target with all arms. However, XSHOOTER is a medium resolution instrument. The individual slit spectrographs have resolutions of $R_{\max }=9,900$ for the UVB arm, $R_{\max }=18,200$ for the VIS arm and $R_{\max }=10,500$ for the NIR arm. In contrast, CARMENES will be fiber-fed providing a resolution of $\mathrm{R}=82,000$. XSHOOTER is also lacking the temperature and pressure stabilization of CARMENES. Another difference between both instruments is the wavelength coverage: While CARMENES is optimized for the wavelength range between 550 and $1700 \mathrm{~nm}$, XSHOOTER covers the whole wavelength range between 300 and $2480 \mathrm{~nm}$.
Despite its medium resolution, XSHOOTER is a good tool for checking the information content that can be expected in these wavelength regions, since there is no other instrument available that covers both the infrared and the visual part of CARMENES. The only other source for spectra of M-type stars (the main target of the CARMENES survey, see Chapter 3) over the whole CARMENES wavelength range are synthetic spectra, e.g. using the PHOENIX code from Hauschildt et al. (1997), a stellar and planetary atmosphere code. PHOENIX spectra can be used to predict the information content of a particular part of the spectrum, depending on the resolution of the spectrograph used. In the case of CARMENES such synthetic spectra have been used in order to evaluate the RV-precision which can be achieved in the NIR, in particularly with respect to different wavelength coverages. However, since no broadband high resolution spectrograph exists in the NIR yet, it is not clear how good the predictions using the PHOENIX model are.

This Chapter shows exemplary how XSHOOTER-spectra of various M-type stars look compared to PHOENIX spectra (binned to a resolution of $\mathrm{R}=10,000)$. While a full catalogue paper is in the works, the following sections give an overview over the obtained spectra, the data reduction, the atmospheric modelling and the agreement between observation and model. 


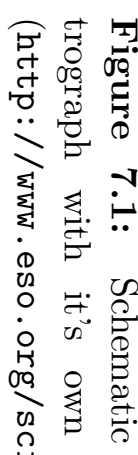

家哭?

号.

苗

施势

总志

为罚

? 8

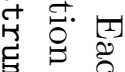

品。

的志声

雚资

苍战

ه

诂.

造造

它廷

官

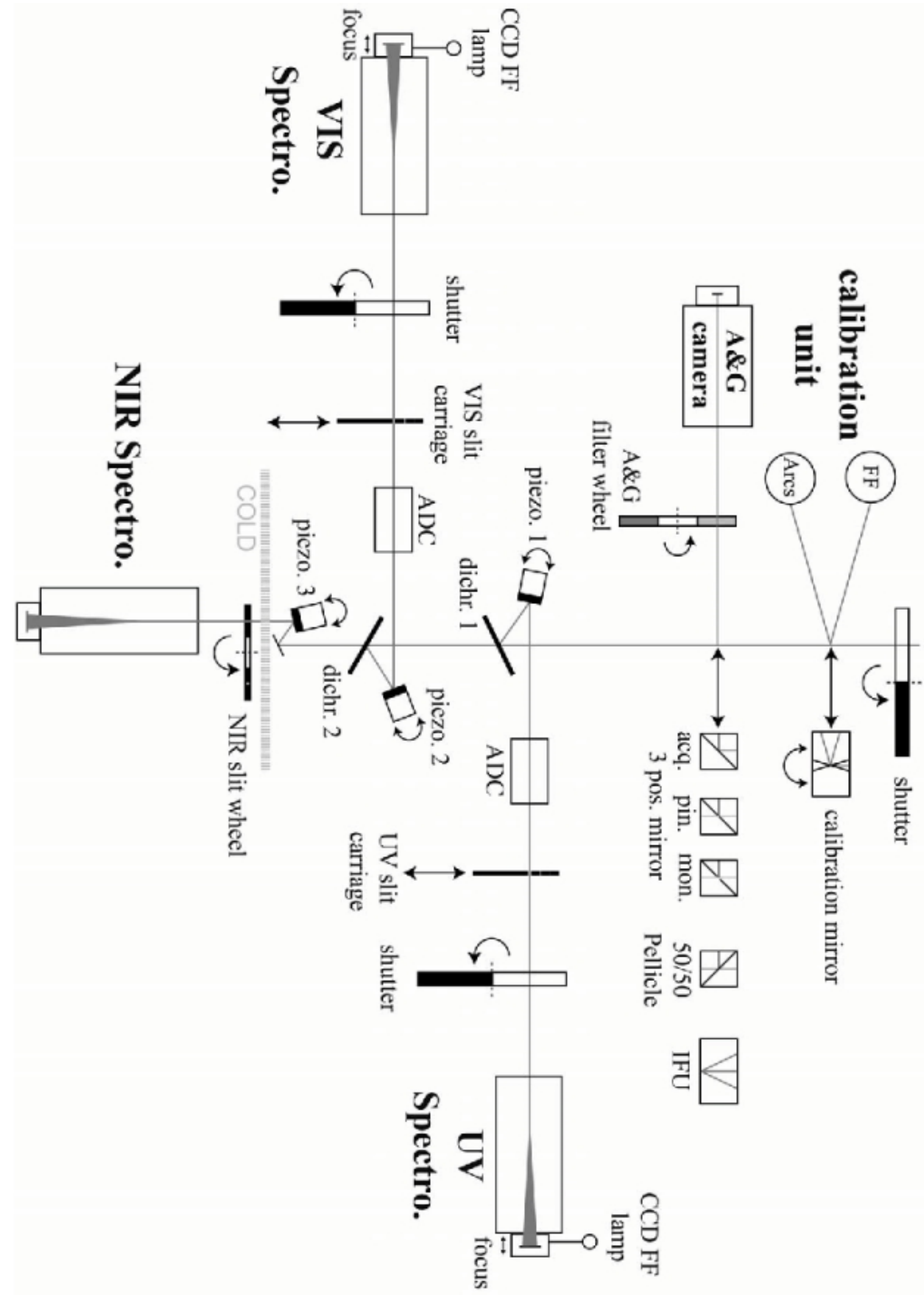




\subsection{Observations and data re- duction}

The observations have been carried out under the ESO program 385.D-0200(A) ("The Complete Picture of Low-Mass Stars from UV to IR: Chromospheric Structure and Consequences for Habitability"), the observed targets are listed in Table 7.1. Additionally we included nine targets from the public ESO archive, observed in a program by Groot et al. ${ }^{1}$ With the exception of GJ 3622, which has been observed using the nodding mode, all objects have been observed in the so-called staring mode.

The X-Shooter spectra have strong order curvature and a varying slit line tilt across the orders. The data reduction was performed with the ESO X-Shooter pipeline ${ }^{2}$ (version 1.3.7). In short, it includes non-linearity correction, bias correction, bad pixel masking, flat-fielding, wavelength calibration, correction for the sky background, optimal extraction, ordering merging, and correction for instrument efficiency and atmospheric extinction.

\subsection{Removing telluric absorp- tion}

In order to retrieve as much useful information from the observations as possible, the absorption lines caused by the Earth's atmosphere have been fitted and removed from the spectra. A detailed description of the method, based on the LBLRTM ${ }^{3}$ (Line-By-Line Radiative Transfer Model) by Clough et al. (1992), can be found in Seifahrt et al. (2010). Husser and Ulbrich (2013) show how the approach of Seifahrt et al. can be used for full-spectrum fitting. Husser and Ulbrich applied their method to all XSHOOTER spectra of this project and

1 These objects are GJ 229a, GJ 273, GJ 285, GJ 328, GJ 381, GJ 382, GJ 393, GJ 406 and GJ 447 from the ESO program 084.D-0795 ("X-Shooter on Cataclysmic Variables and white dwarfs").

2 http://www.eso.org/sci/software/pipelines/

3 http://rtweb.aer.com/lblrtm_frame.html
Table 7.1: Listed are the spectral type (SpT), visual magnitude $V$, and distance $d$.

\begin{tabular}{cccc}
\hline \hline Star & SpT & $V[\mathrm{mag}]$ & $d[\mathrm{pc}]$ \\
\hline GJ 588 & M2.5 & 9.311 & 168.66 \\
GJ 674 & M3V & 9.407 & 220.24 \\
GJ 701 & M2.0V & 9.360 & 128.89 \\
GJ 735 & M3.0V & 10.194 & 84.94 \\
GJ 832 & M1.5 & 8.672 & 201.87 \\
GJ 849 & M3.5V & 10.366 & 109.94 \\
GJ 876 A & M5.0V & 10.192 & 213.28 \\
GJ 880 & M2.0V & 8.638 & 146.09 \\
GJ 889.1 & M0 & 10.887 & 63.06 \\
GJ 908 & M2V & 8.993 & 167.29 \\
GJ 3622 & M6.0V & 15.60 & 221 \\
GJ 273 & M3.5V & 9.872 & 262.98 \\
GJ 285 & M4.5V & 11.225 & 167.88 \\
GJ 328 & K7 & 9.98 & 0 \\
GJ 381 & M2.5 & 10.629 & 81.08 \\
GJ 382 & M2.0V & 9.256 & 127.08 \\
GJ 393 & M2.5V & 9.650 & 141.5 \\
GJ 406 & M6.0V & 13.507 & 418.3 \\
GJ 447 & M4.5V & 11.153 & 298.04 \\
\hline
\end{tabular}

the resulting spectra, corrected for the atmospheric features, are shown in Sec. 7.5.

\subsection{Spectral line identification}

In order to identify the atomic and molecular absorption lines in the spectra off all stars automatically, the newest version of the VALD database (Kupka et al., 2000) is used: the VALD3 beta release. Due to the relatively low resolution of XSHOOTER compared to CARMENES it has not been the intention to actually fit the observed spectra but rather to get an overview on the lines available for RV-measurements. Therefore only one stellar spectrum has been extracted from VALD, us$\operatorname{ing} T_{\text {eff }}=3500 \mathrm{~K}, \log g=5$ and the lowest Microturbulence accepted by VALD $\left(0.01 \mathrm{~km} \mathrm{~s}^{-1}\right)$, representing a typical M2 star.

The VALD line list consists of around 44,000 lines between 350 and $2500 \mathrm{~nm}$, including both 
atomic and molecular absorption lines. For each line VALD provides the ionization state, the wavelength and the line depth. There is also additional information available, e.g. about the damping parameters, Stark and Waals factors, which is not used in this work.

For each $100 \mathrm{~nm}$ chunk of the stellar spectra the deepest lines have been selected from the list in order to visualize the most prominent features. Since the density of the lines per chunk is varying for the different wavelength regions, the thresholds used are adjusted in order to provide high readability of the plots ${ }^{4}$.

\subsection{Results}

Figures 7.2 to 7.22 show the spectra of three M-type stars: GJ889.1 (M0), GJ735 (M3) and GJ3622 (M6.5). On the very top TiO (blue) and $\mathrm{FeH}$ (red) lines are indicated above the line identification of other atomic and molecular lines. Next, a PHOENIX spectrum, downscaled to $\mathrm{R}=10,000$ is shown (dark blue). The full resolution PHOENIX spectrum can be seen in the background (light blue). Below, in black the reduced and flux calibrated observation of GJ889.1 (M0) is plotted. An example spectrum of the Earths atmosphere is shown in green and the bottom three lines show the spectra of the three M-type stars with the atmospheric correction, as described in Sec. 7.3, applied.

In regions with very strong atmospheric absorption (e.g. between 1850 and $1950 \mathrm{~nm}$ ) the corrected spectra are plotted with dotted lines to indicate that the resulting spectrum does not contain any stellar information and is dominated by the Earths atmosphere.

For the first two chunks $(350-450 \mathrm{~nm}$ and $450-550 \mathrm{~nm}$ ) the lines selected from the VALD line list by the method described in Sec. 7.4 are not representing the strongest lines of the observed spectra. This is probably caused by

4 Thresholds of the line depth for the chunks from blue to red: $0.997,0.975,0.9,0.7,0.6,0.5,0.3,0.3,0.5,0.5$, $0.99,0.4,0.3,0.2,0.99,0.6,0.7,0.25,0.4,0.45,0.5$ the vast number of lines present in these regions combined with the relatively low resolution of the observed spectra. The deepest single lines do not necessarily show up as the strongest features in the observed spectra since a very dense forest of weaker lines can merge into one big feature. Identifying the strongest features in these regions would require line identification by hand or a more appropriate line list.

Overall, the PHOENIX model and the XSHOOTER spectra match fairly well and a very large fraction of lines from the VALD line list can be found with similar intensities in both the observations and the model. There are a few noticeable exceptions: For example the Li 1 double-lines at $670.79 \mathrm{~nm}$ (see Fig. 7.5) are very strong on the PHOENIX data, however they are barely visible in the observations. In contrast the Ca 1 lines at $671.77 \mathrm{~nm}$ and at $714.82 \mathrm{~nm}$ are much stronger in the observations than in the model. There are also some trends visible in between the different M-type stars. For example the Fe1 and Ca1 lines between $850 \mathrm{~nm}$ and $870 \mathrm{~nm}$ (see Fig. 7.7) get weaker from early- to late-type stars while the Fe 1 and K 1 lines between $1150 \mathrm{~nm}$ and $1250 \mathrm{~nm}$ (see Fig. 7.10) grow stronger from early to late-type stars. 


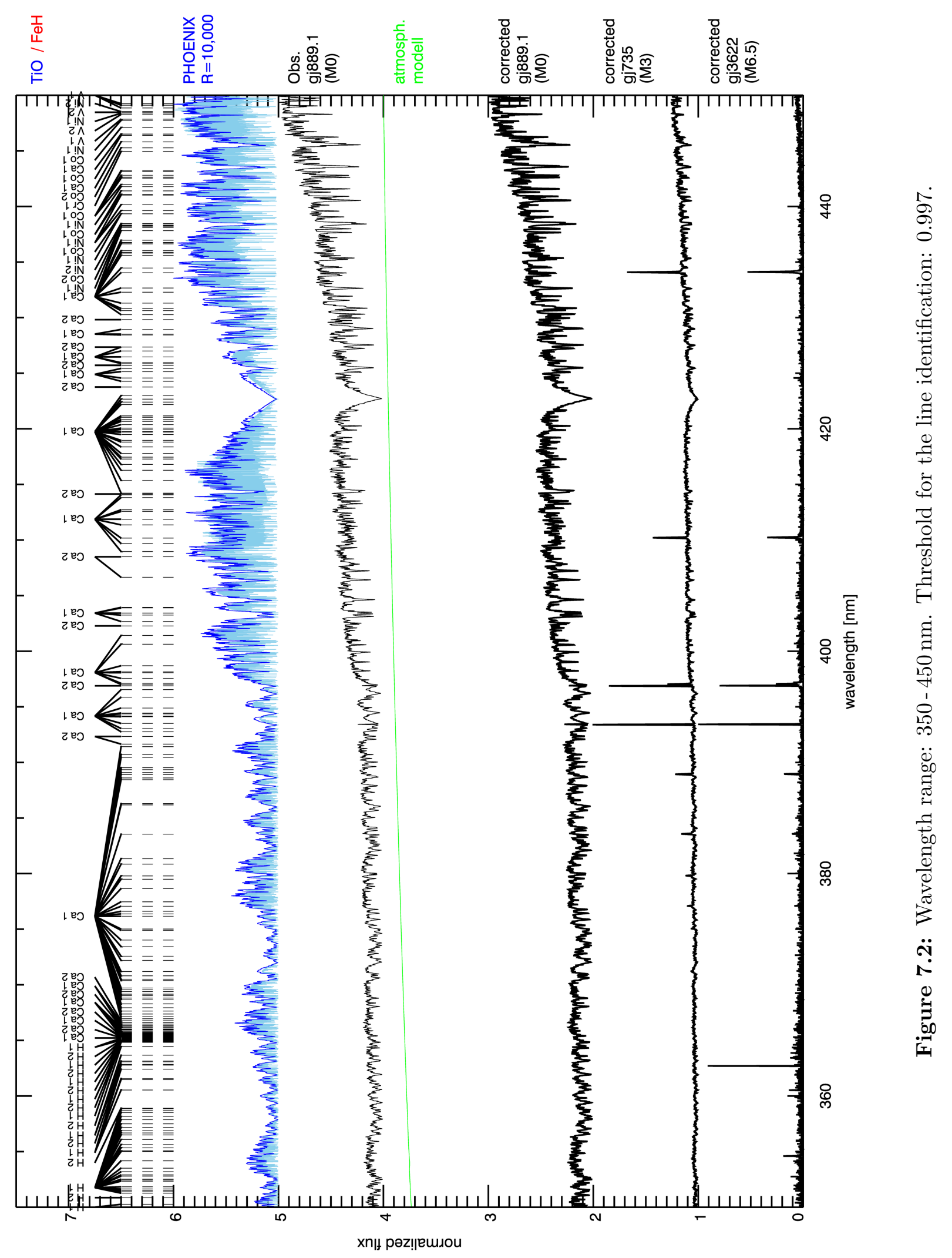




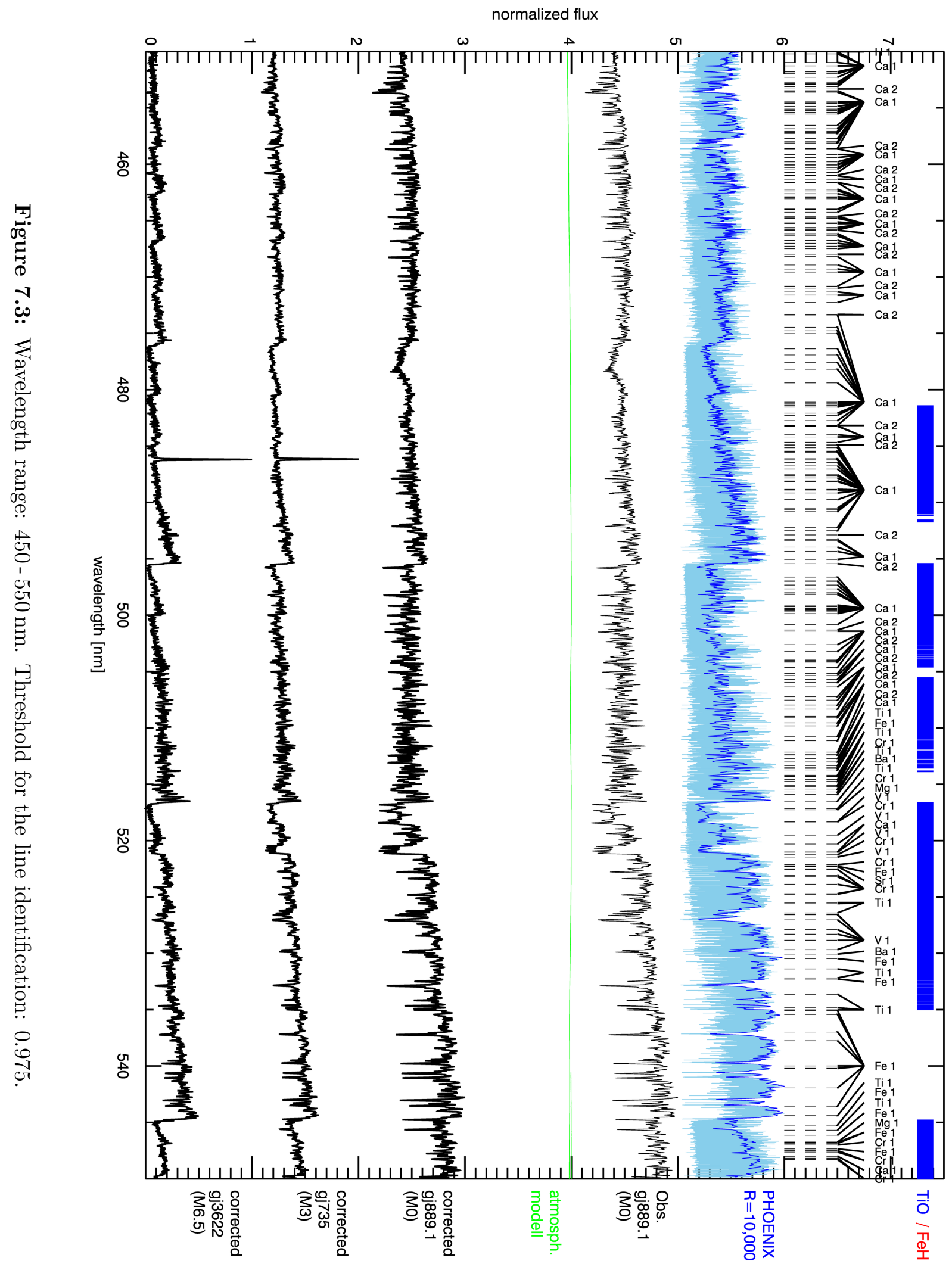




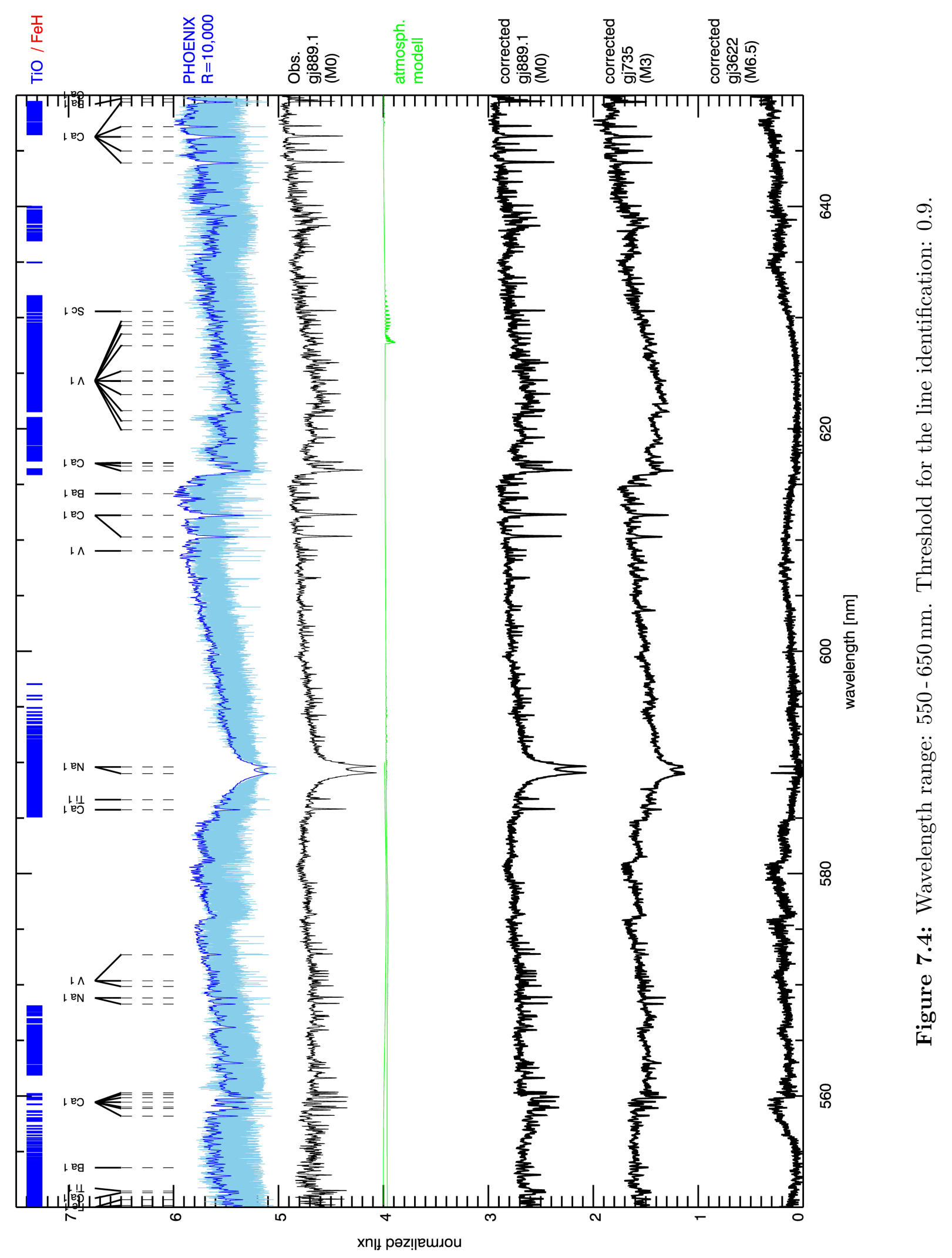




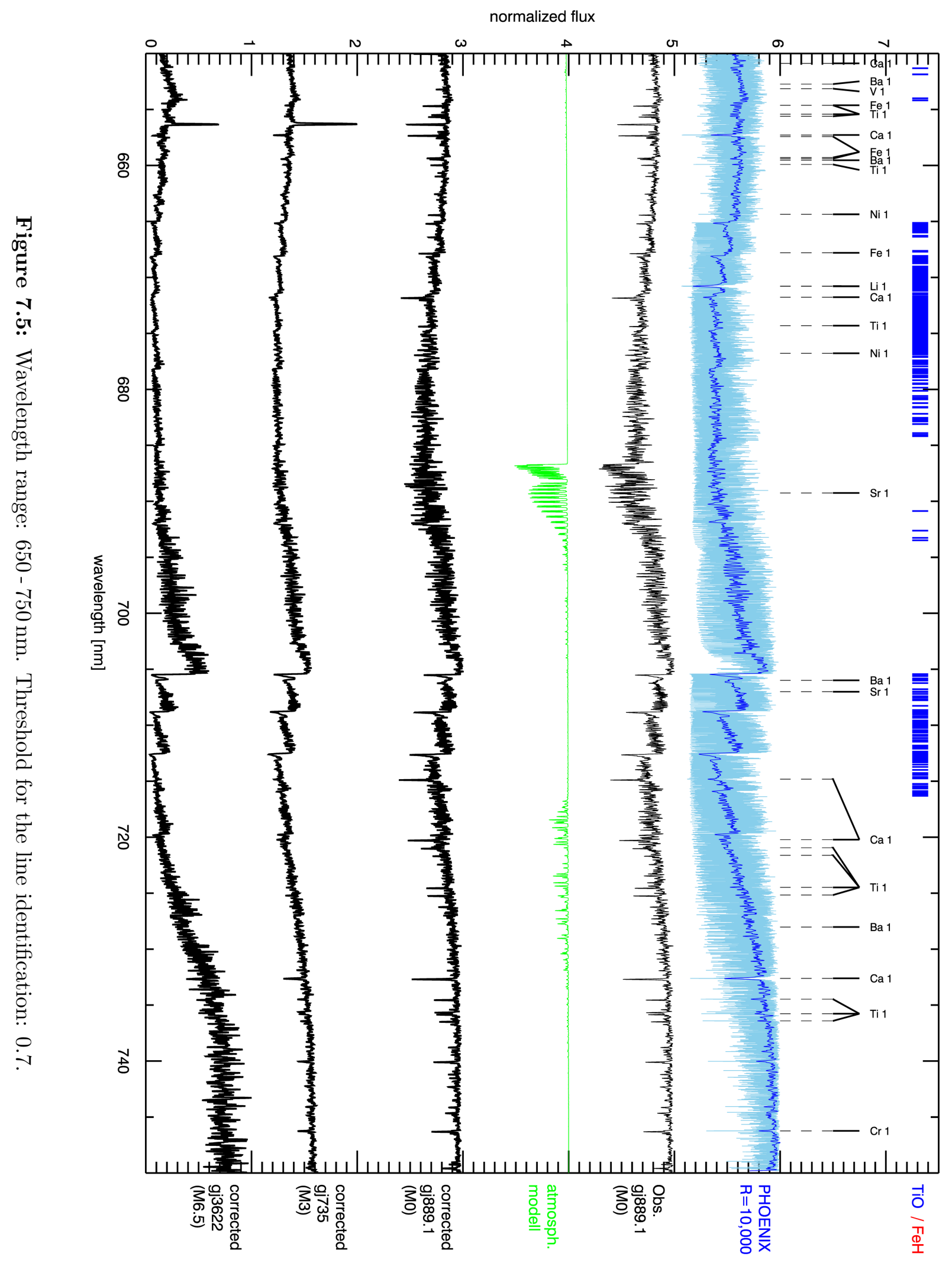




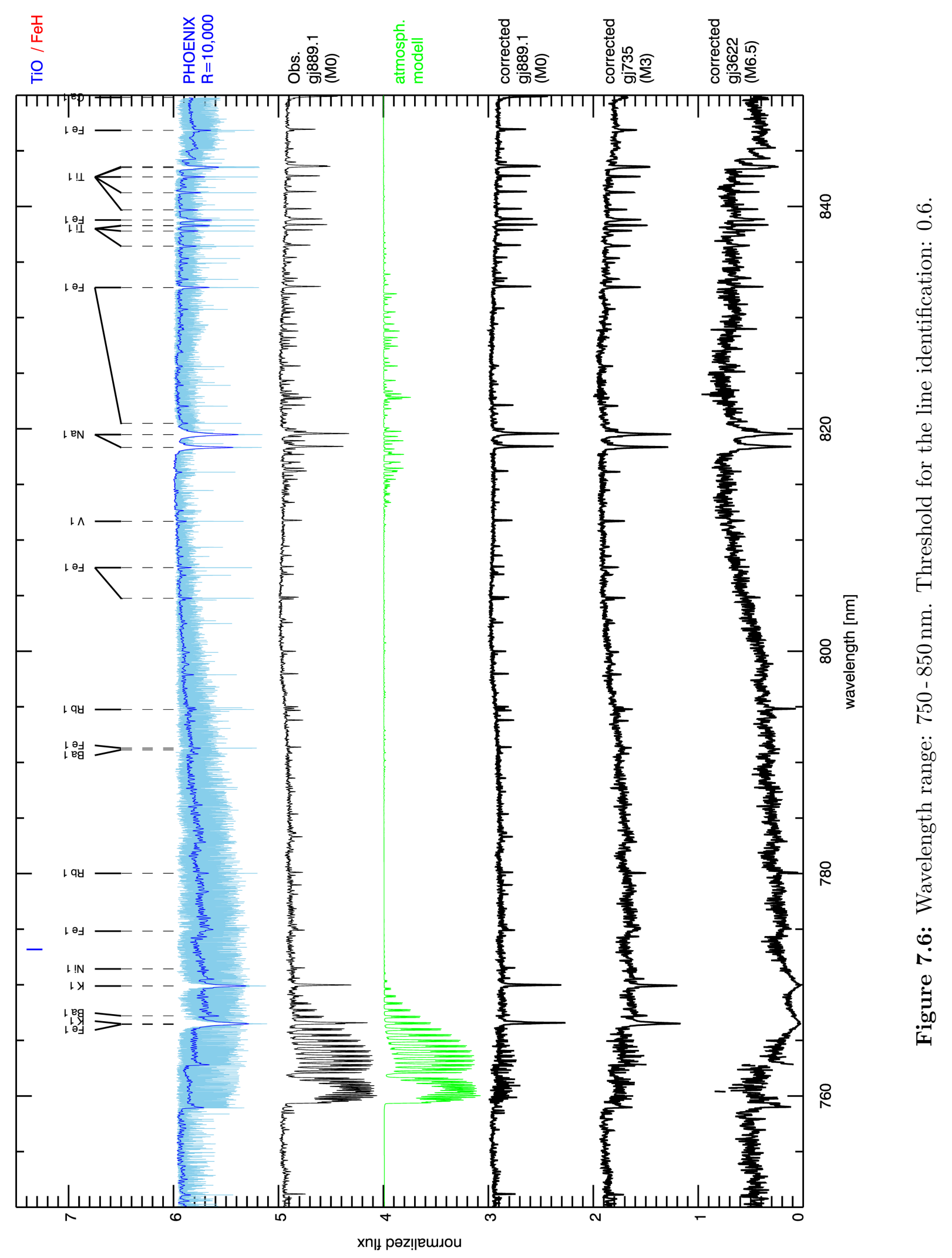




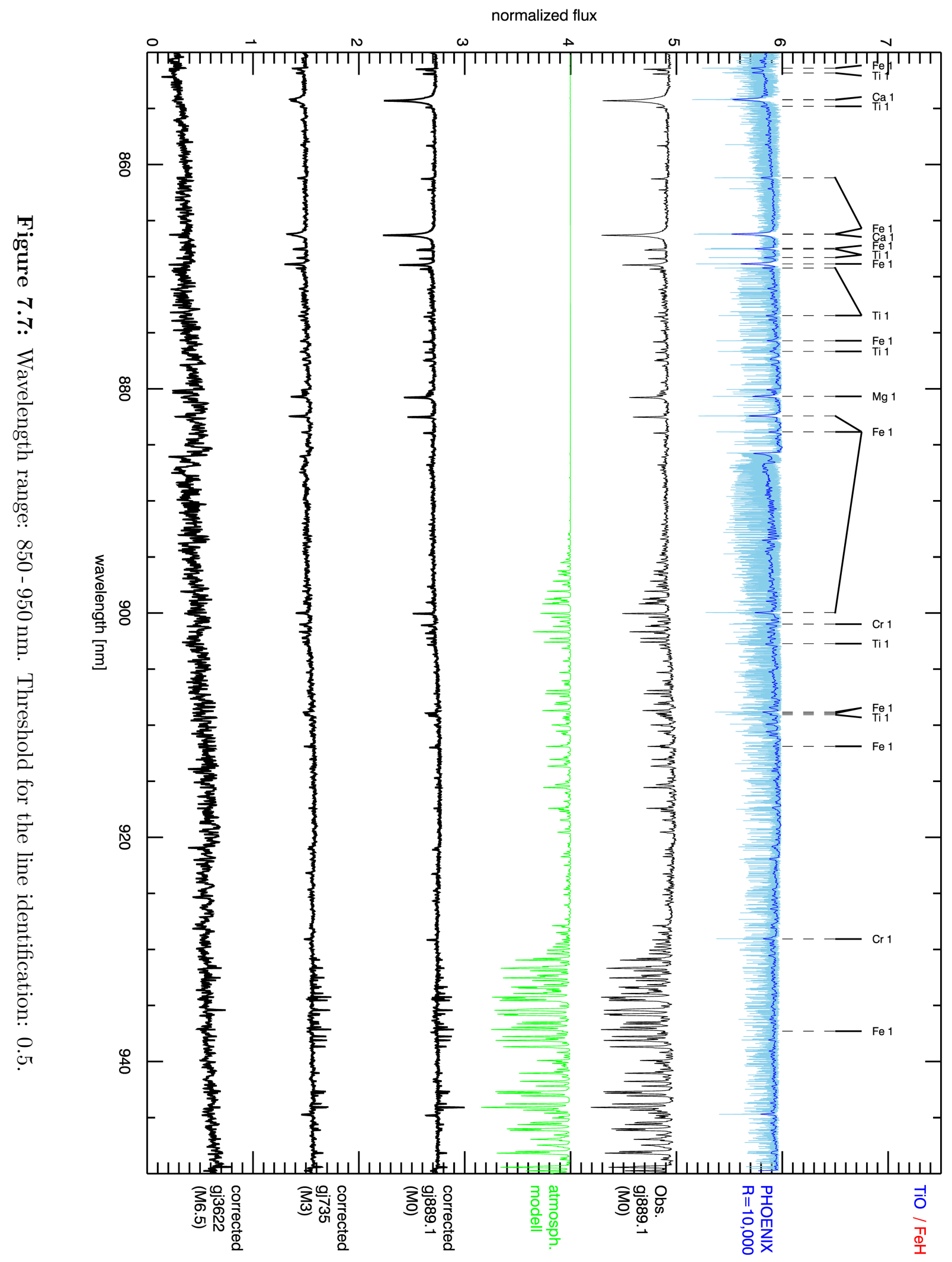




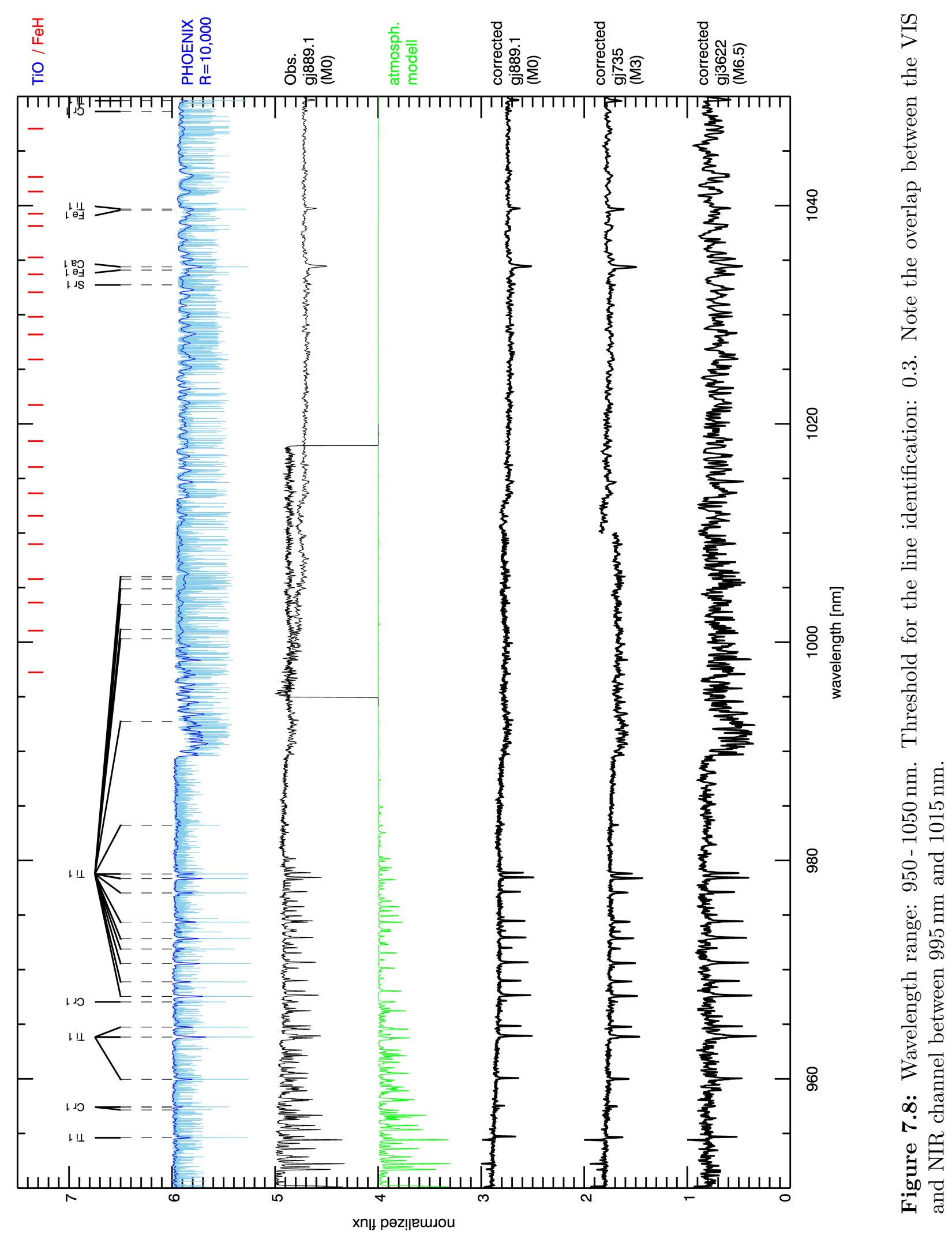




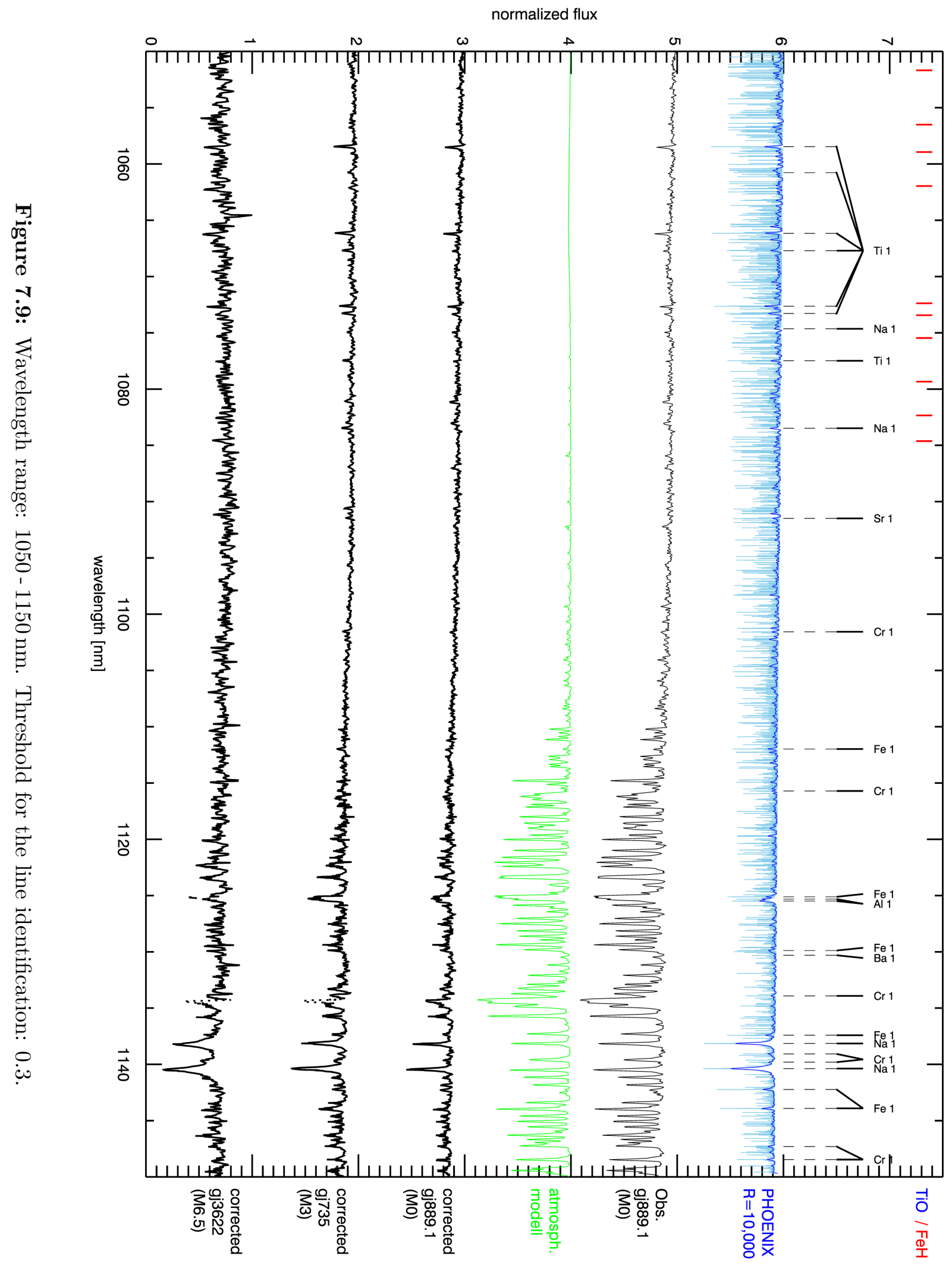




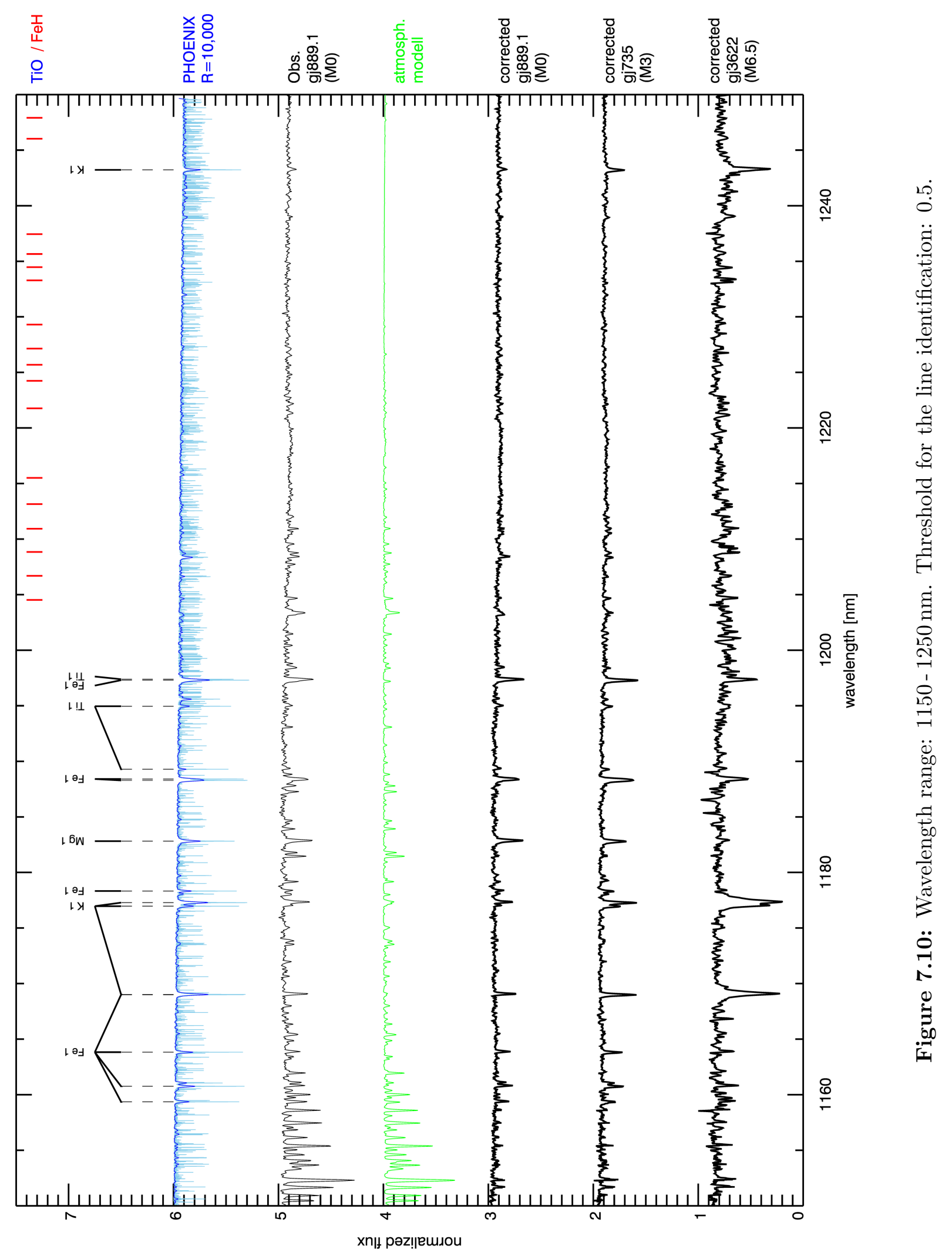




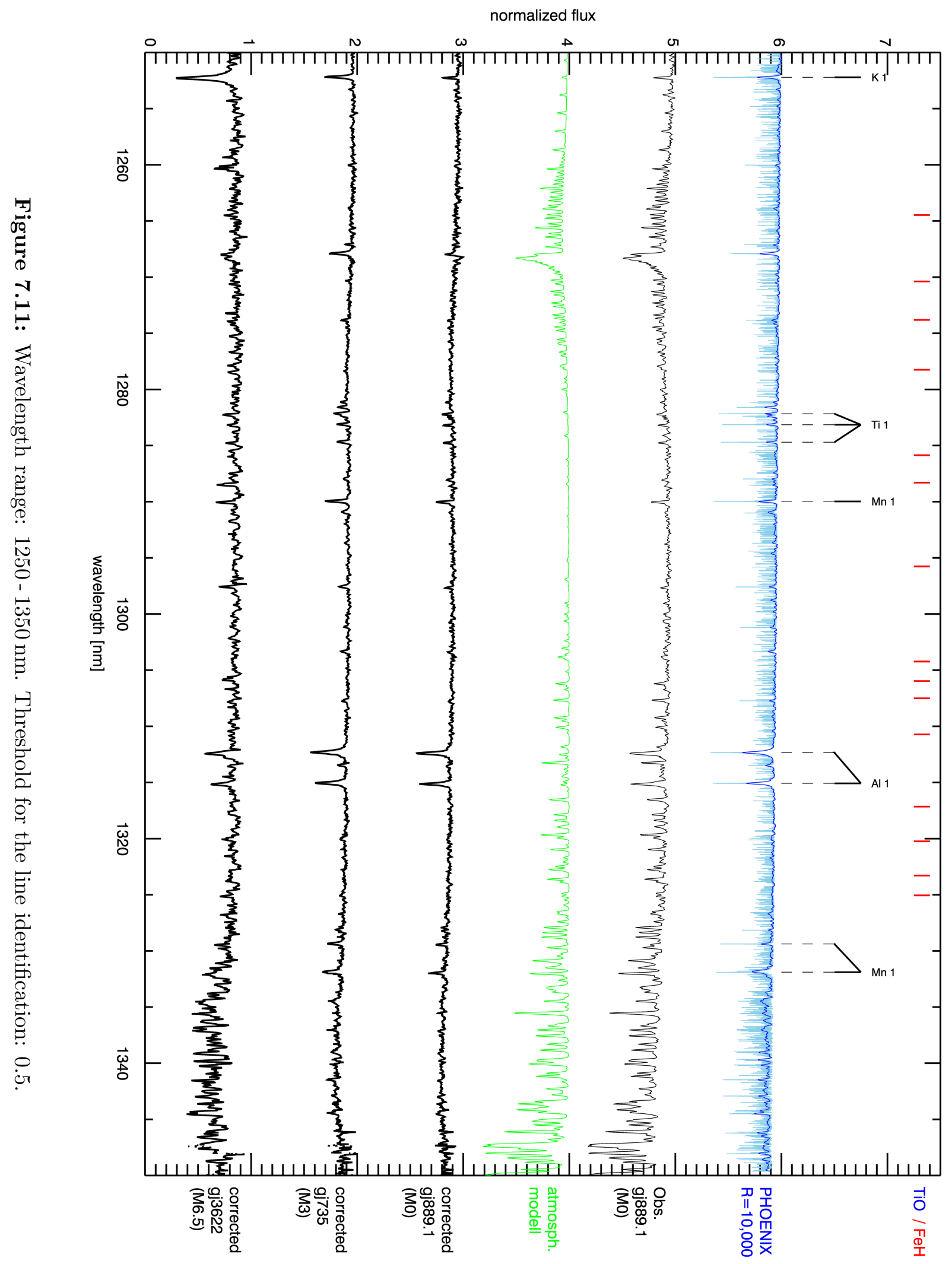




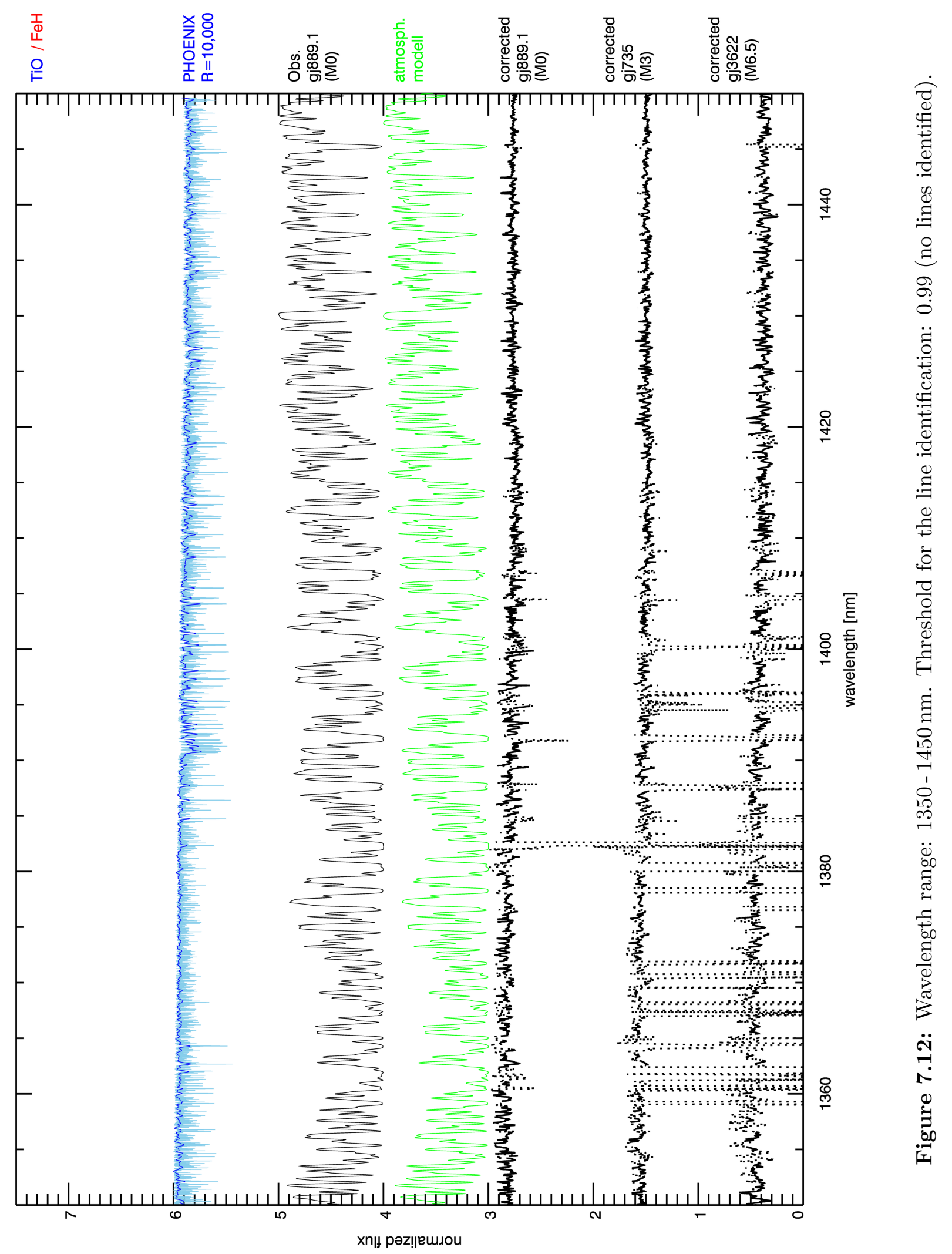




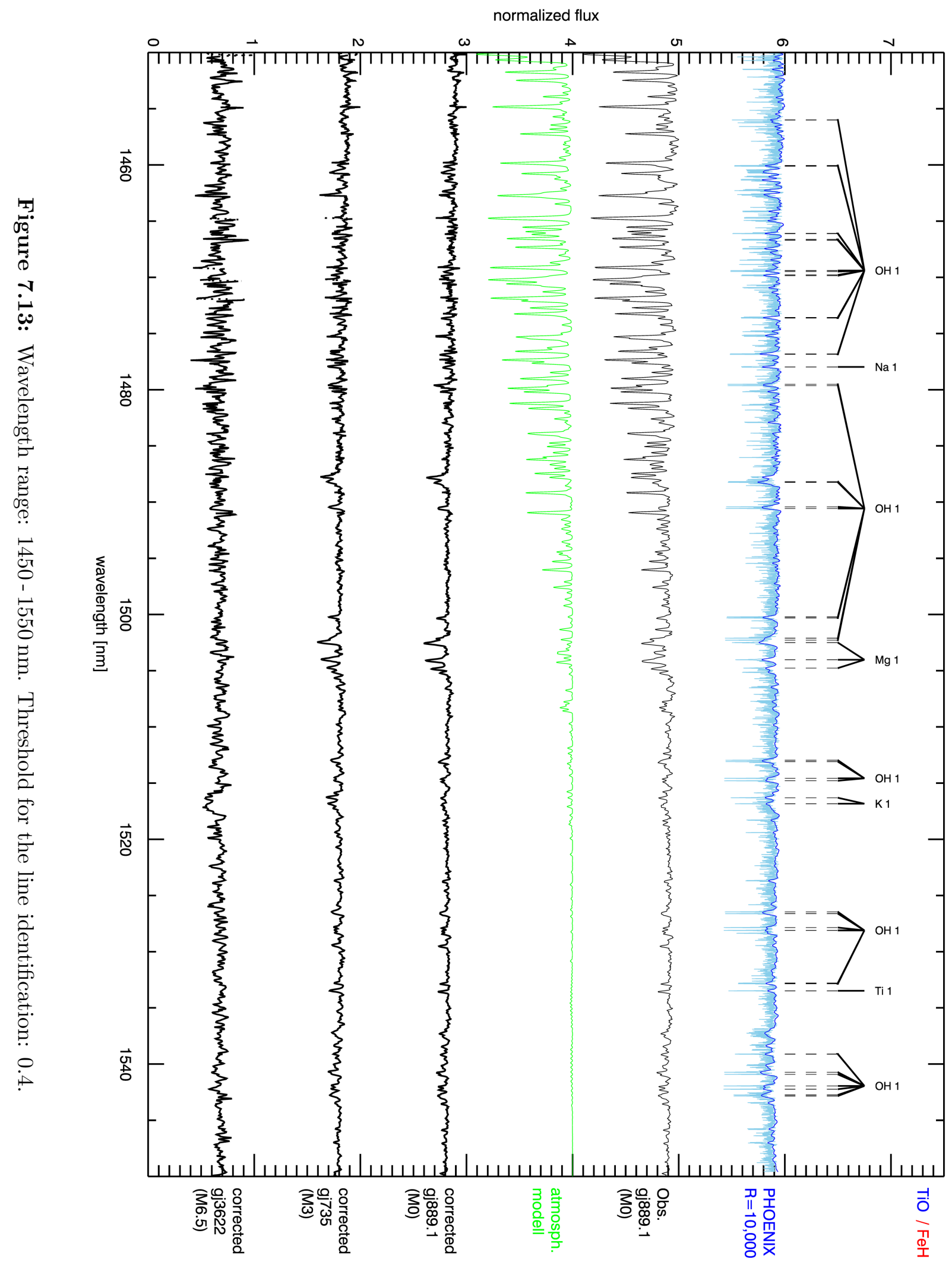




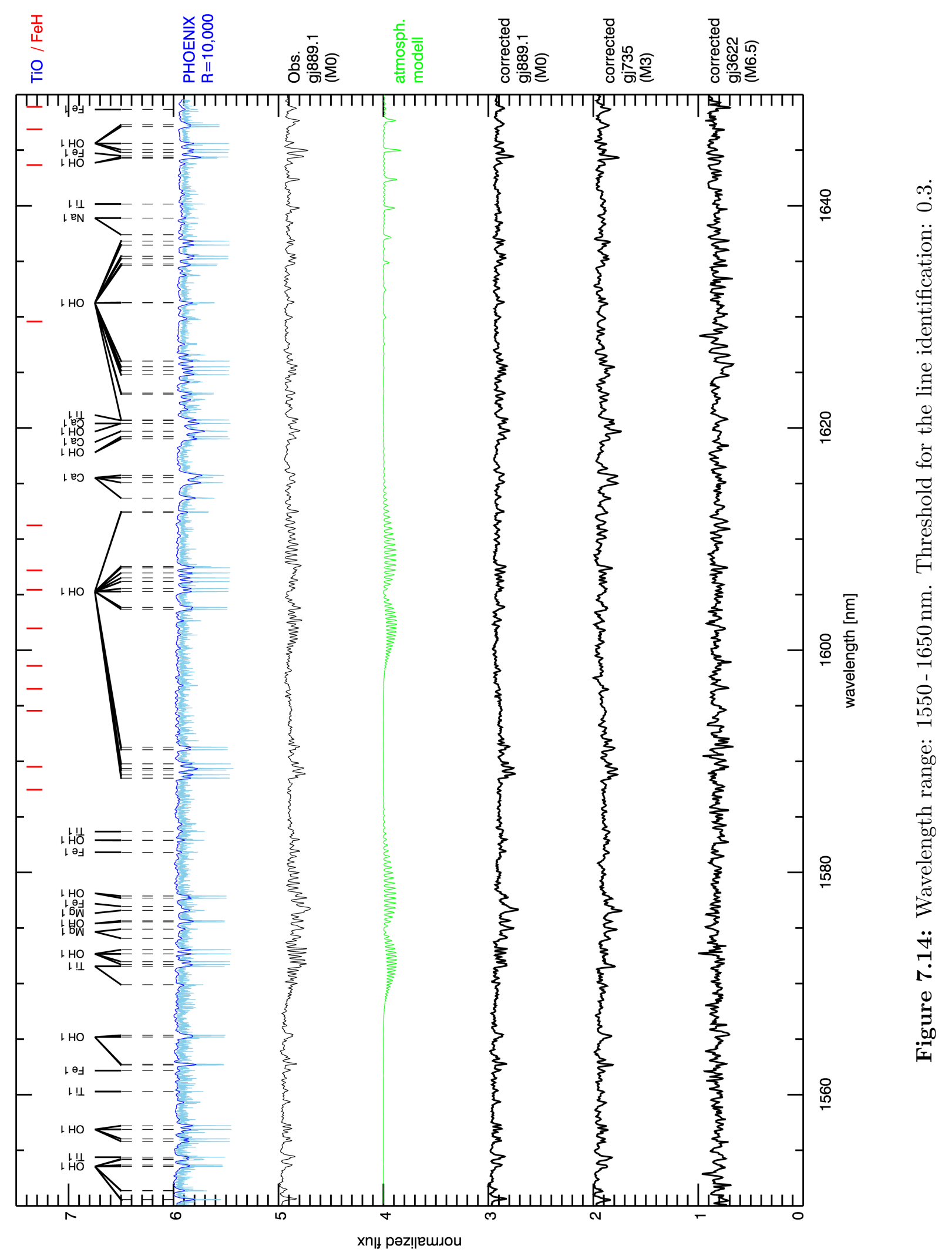




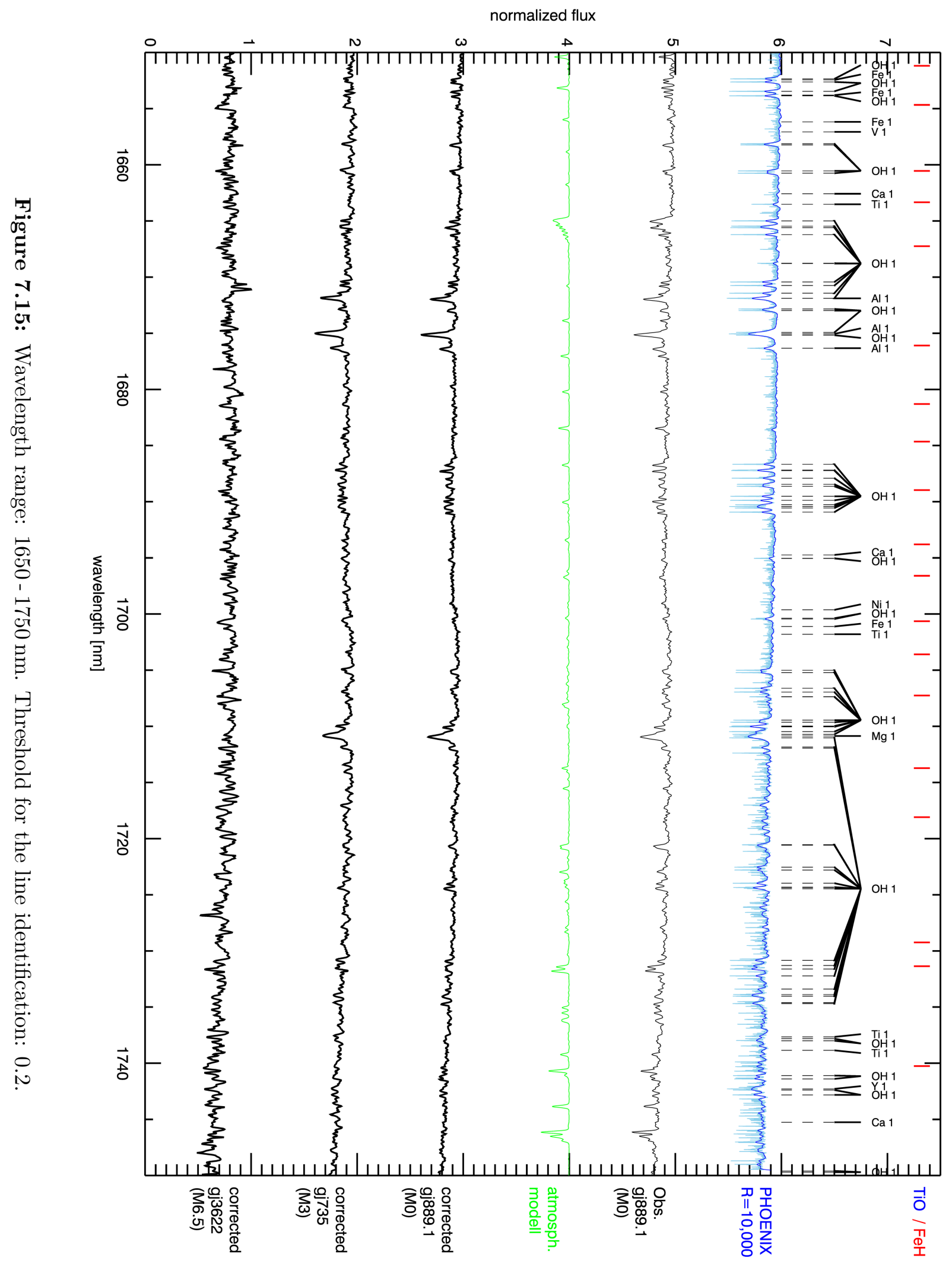




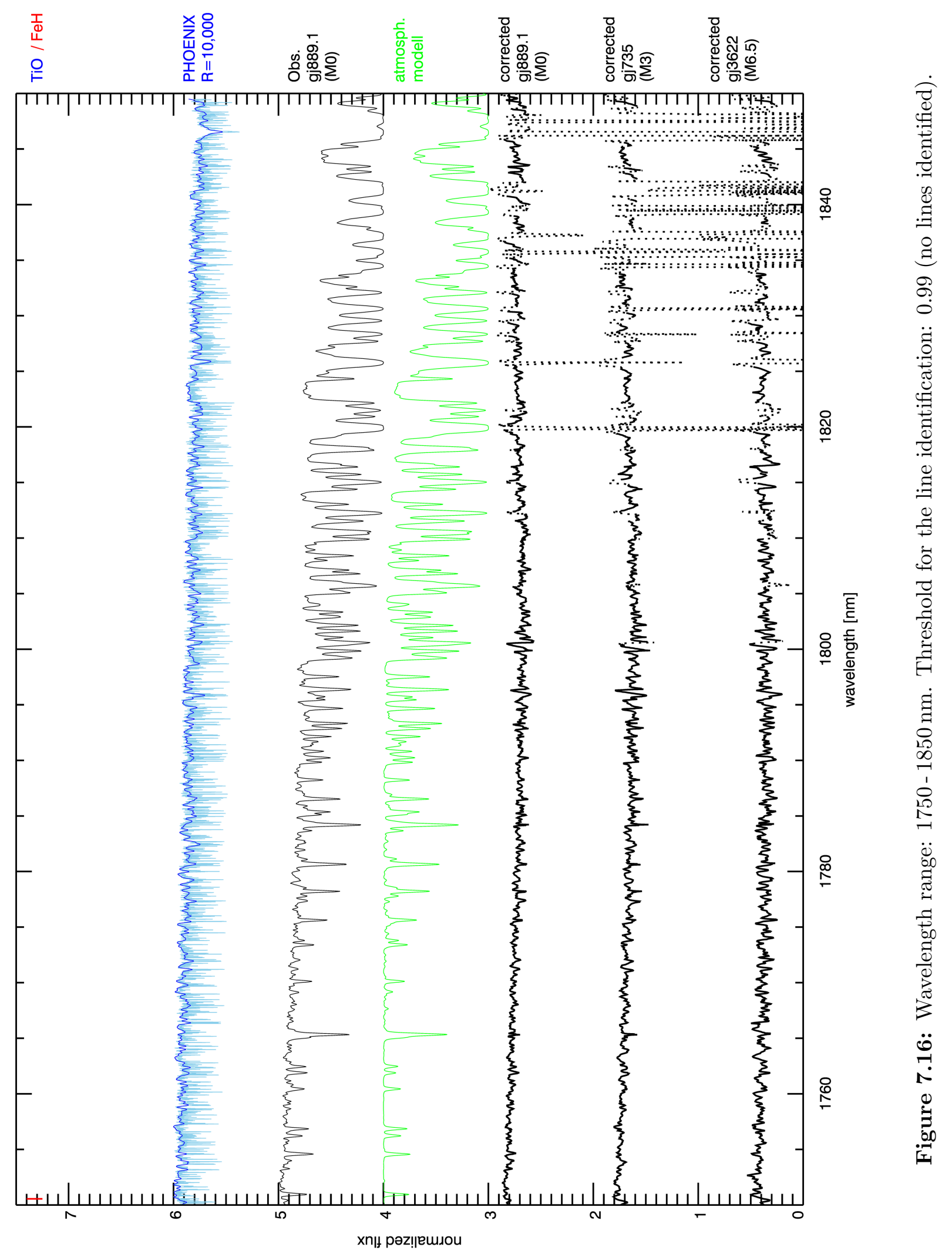




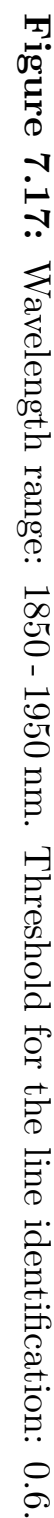

ळ্口
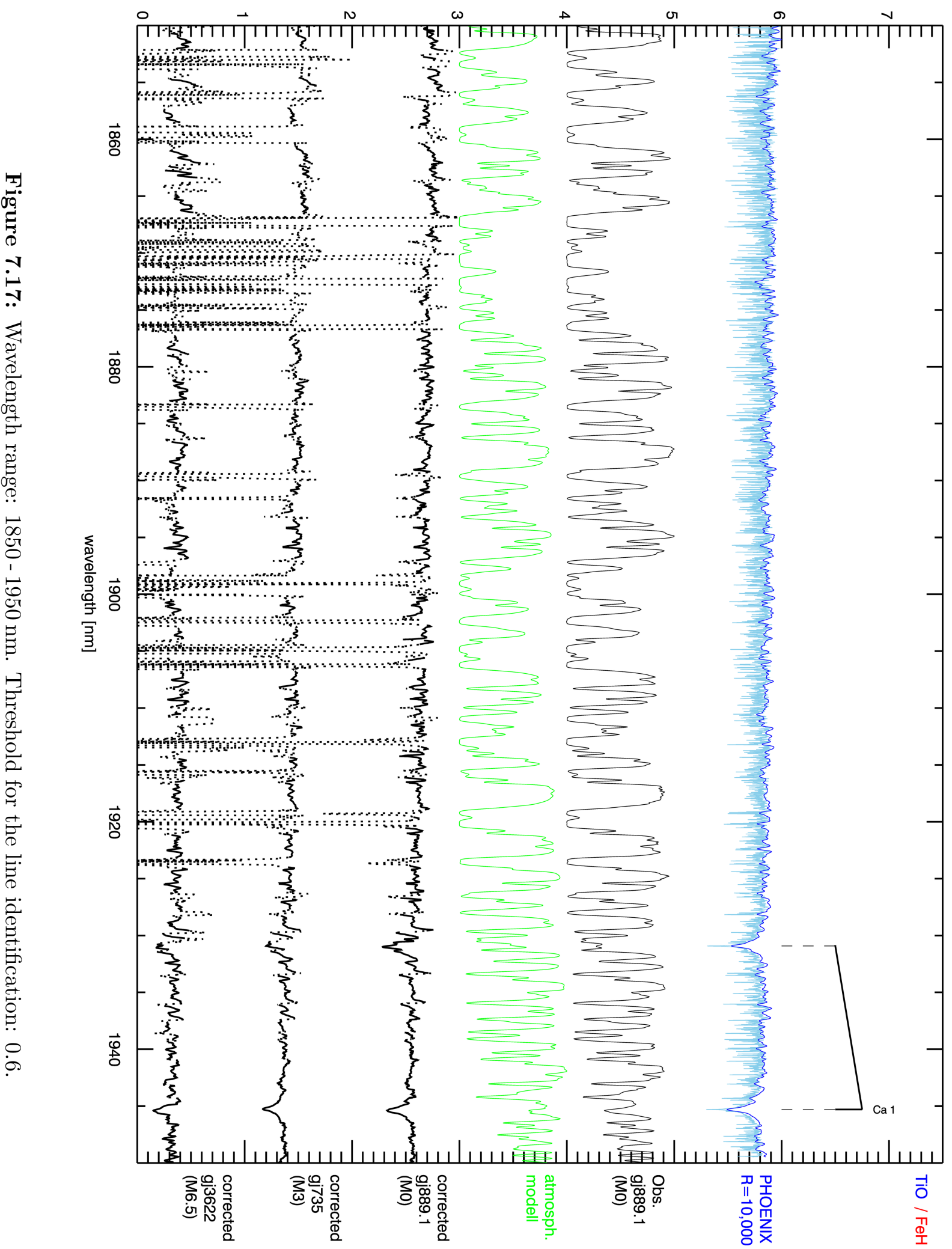

normalized flux 


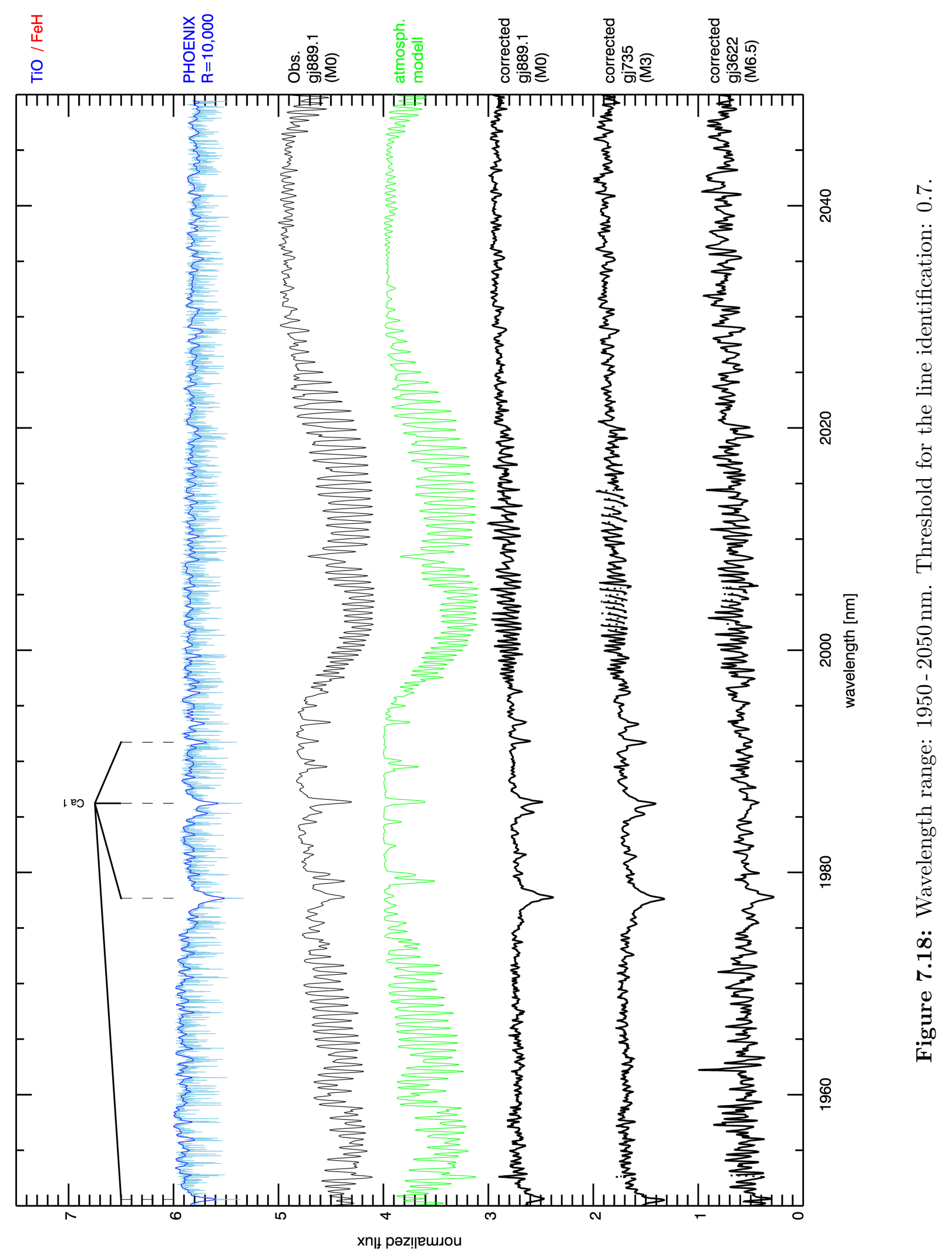




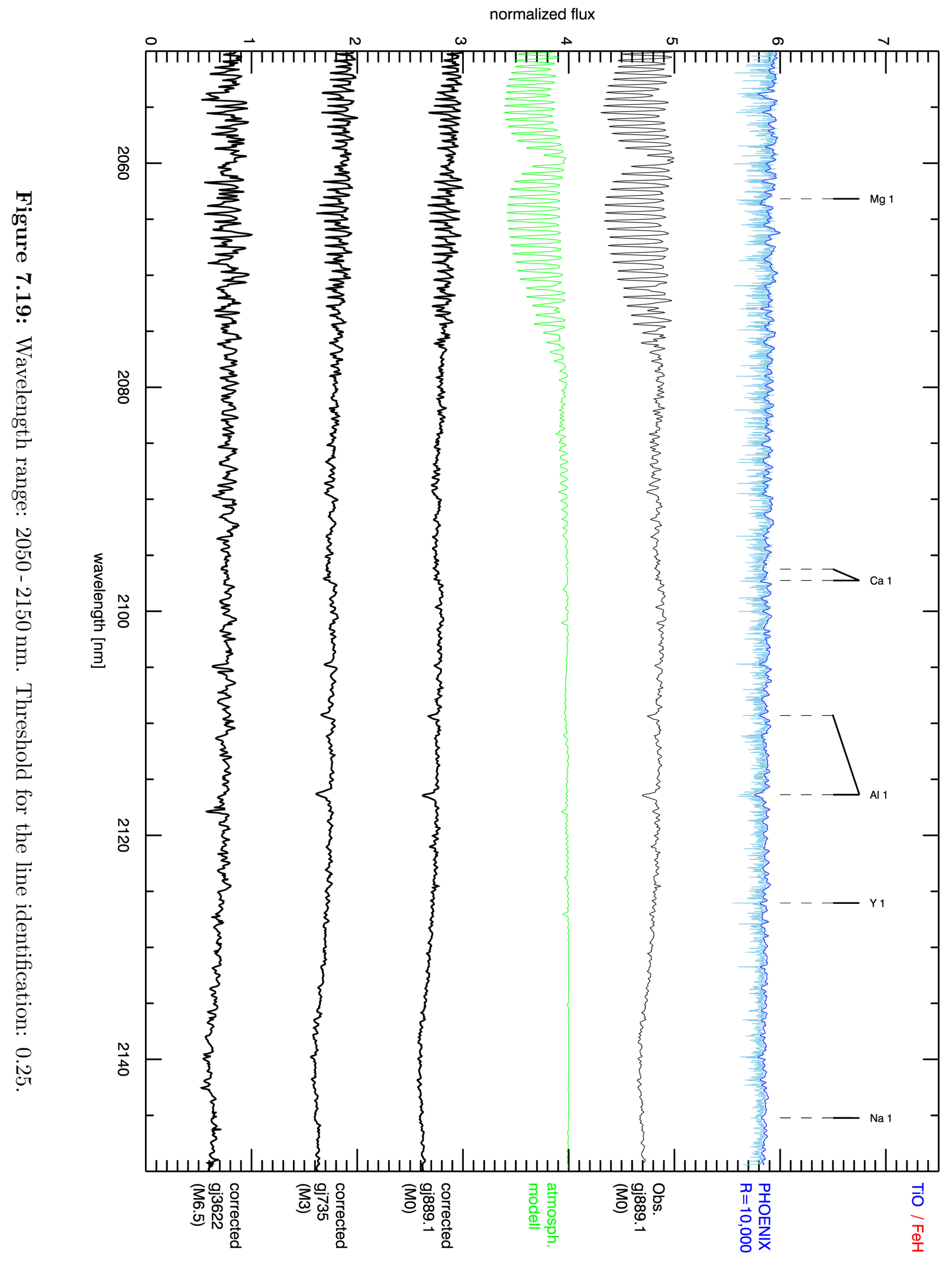




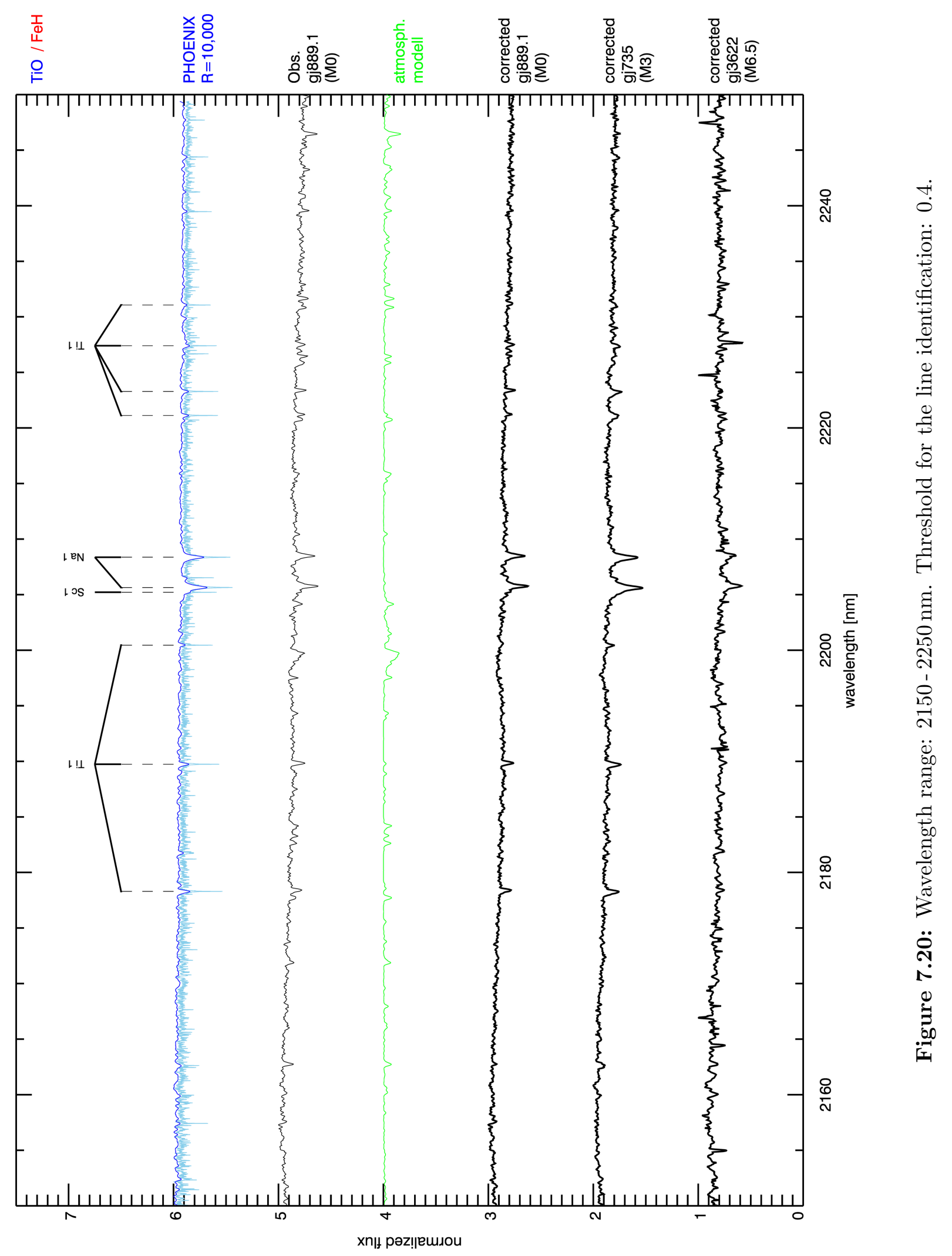




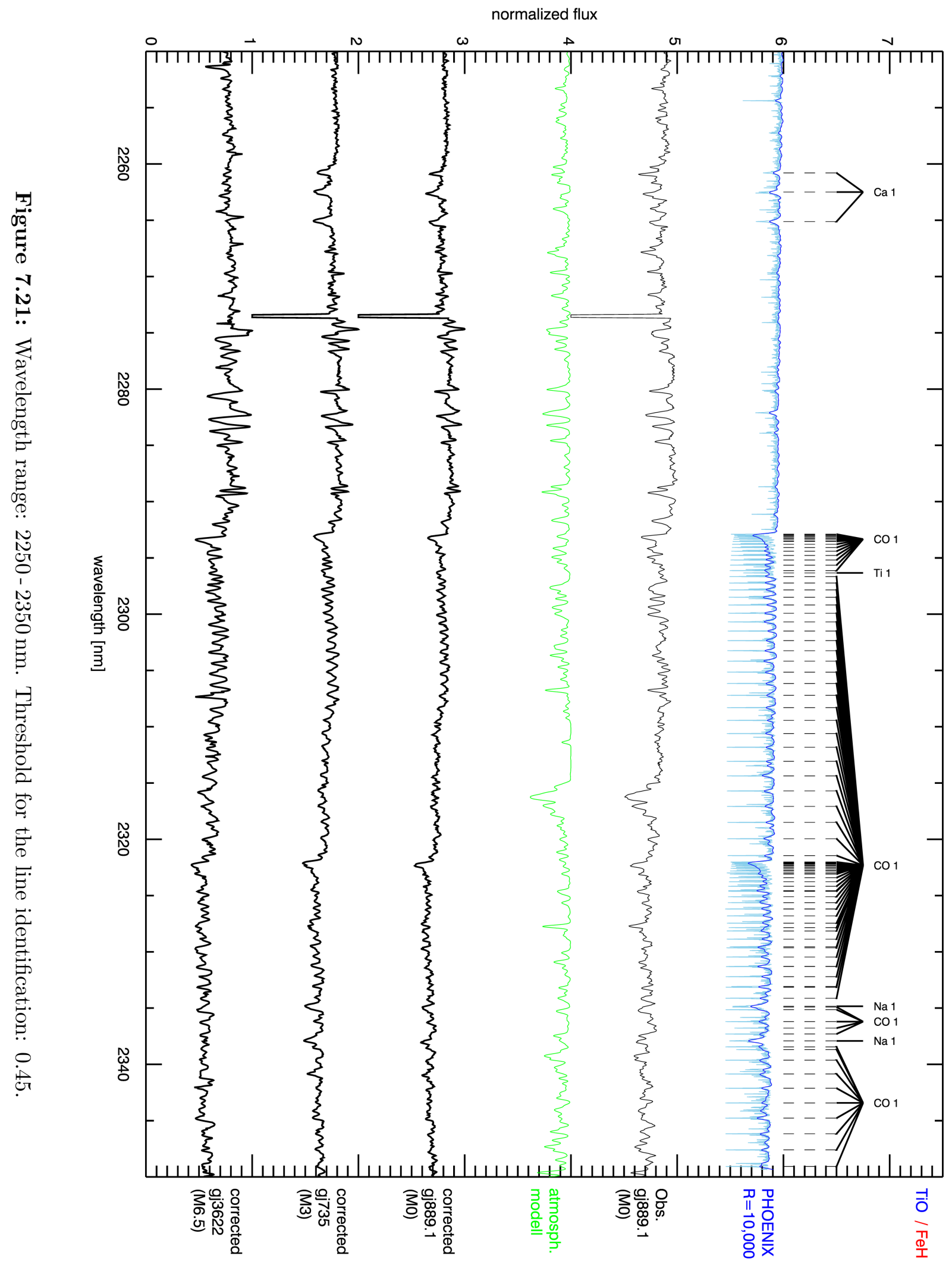




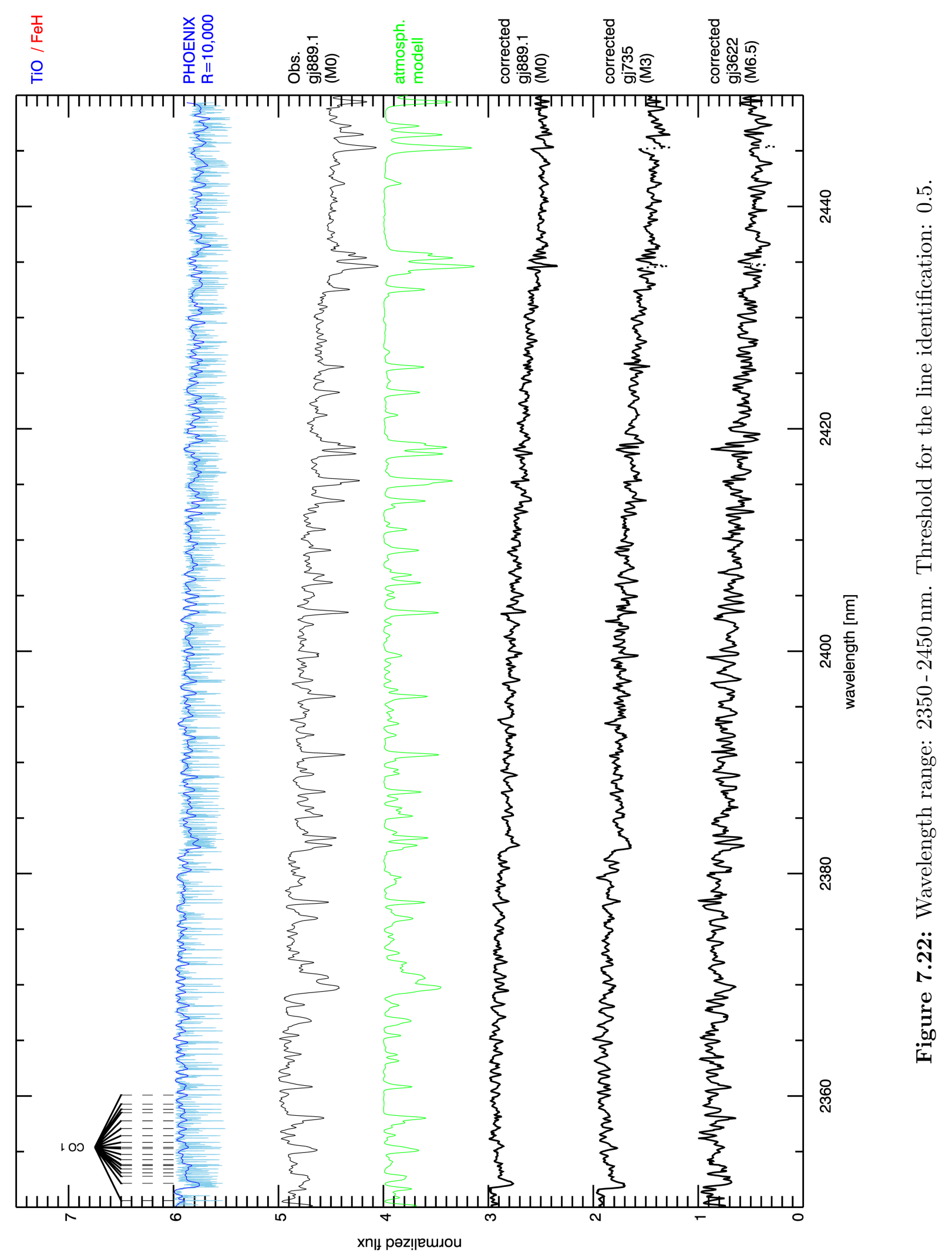




\section{Chapter 8}

\section{Conclusion \& Outlook}

In this work a FP unit for high precision RV calibration on the $10 \mathrm{~cm} \mathrm{~s}^{-1}$ level, from the first simulations to the final setup to be used at CARMENES is developed. CARMENES is the next generation high resolution spectrograph which is currently being built for the $3.5 \mathrm{~m}$ telescope at Calar Alto.

Another part of this work involved using XSHOOTER spectra to create a stellar atlas of M-type stars, including atomic and molecular line identification and the removal of the Earth's atmosphere using an atmospheric model. XSHOOTER's uniquely large wavelength coverage from $350 \mathrm{~nm}$ to $2450 \mathrm{~nm}$ enables for the first time an overall view on M-star spectra from the visual to the near infrared.

As a first step of the FP project, simulations of the influence of temperature, pressure and mechanical stability on the resulting RV error of a theoretical FP have been made. These simulations confirmed the principle feasibility of using a FP unit for RV calibrations on the sub- $\mathrm{m} \mathrm{s}^{-1}$ level and provided first requirements for the temperature and pressure stability. For a $10 \mathrm{~cm} \mathrm{~s}^{-1}$ RV stability the pressure has to be stabilized to $4 \cdot 10^{-3}$ mbar and the temperature must not change by more than $15 \mathrm{mK}$.

A test setup using a cage construction without any further stabilization was built to provide the first spectra of two previously purchased FPs, optimized for the two arms of the CARMENES instrument. This setup has also been used to create the first FTS spectra at very high resolution. The spectra show a number of unexpected features compared to the original simulations, which turned out to be caused by the fibers used to feed the light into the FP.

Extensive simulations of the effect of fibers in combination with FPs were made in order to explain the deviations of the observed spectra from the expected results. The fiber diameter has a direct influence on the contrast of the FP peaks, leading to lower contrast (and therefore a smaller signal to noise ratio) for larger fibers. Similarly, the degree of asymmetry in the lines increases with fiber diameter. Both effects are also influenced by the light distribution of the fibers, which is not constant due to time dependent fiber scrambling. Thus the resulting maximum RV precision of the FP unit is also limited by the stability of the light distribution of the fibers. Using smaller fiber diameters reduces the maximum RV error due to these effects and consequently fiber diameters of $50 \mu \mathrm{m}$ or smaller are recommended for a RV stability of better than $10 \mathrm{~cm} \mathrm{~s}^{-1}$. Alternatively, additional effort has to go in stabilizing or at least monitoring of the light distribution.

The final setup for the FP unit has been designed in order to fulfill the requirements for a $10 \mathrm{~cm} \mathrm{~s}^{-1}$ RV stability. It consists of a double layered vacuum tank with the optics hold in place by blocks of Aluminum, which are mounted on a massive stage. A liquid coolant (silicon oil) is constantly circulating through the inter-layer of the tank and is kept at a stable temperature by an external cooling circulator device. This setup provides a uniform 
temperature distribution of the whole tank, avoiding any temperature gradients. The large mass of the tank itself adds additional inertia to the whole process. Thus the air inside the tank will be kept at an extremely stable temperature (better than $\pm 15 \mathrm{mK}$ ).

The next step of the FP project will involve verifying the connection between temperature changes and changes in the FP spectrum. In order to do this, spectra with different temperatures of the FP unit will be taken with the FTS. The temperature can be changed by adapting the cooling circulators target temperature. It mainly depends on the stability of the FTS, what the smallest temperature change that is still visible in the spectrum will be. At the moment the FTS is stable enough to show RV deviations as small as $1 \mathrm{~m} \mathrm{~s}^{-1}$ over one night. However, doing long term measurements of the FP to test its stability over weeks and months would be of great value for CARMENES since it could potentially change the calibration strategy of the spectrograph.

There will also be a second FP unit for the near-infrared FP. Its design will be identical with the one already built, with the possible exception of using different fiber materials and sizes because the different detector might change the required flux from the FP unit and therefore different fibers might be better in terms of the signal to noise ratio or RV stability. This second unit will also be tested extensively in the lab before being installed at CARMENES.

Finally, there is a plan to externally track the drift of the FP. While the FP unit described in this work is only passively stabilized by controlling the temperature and pressure, tracking the effective change of the mirror distance caused by these changes would be a huge improvement. The basic idea is to use a Pound-Drever-Hall (PDH) locking scheme (Drever et al., 1983) to lock a laser onto a Rb cell to get an absolute reference wavelength and using a second laser to be locked with the $\mathrm{PDH}$ technique to one of the FP lines. The drift between those two lasers can be measured and directly provides possible changes of the effective FP length. In theory this can be done with extremely high accuracy, resulting in measurements with RV precisions of up to $1 \mathrm{~cm} \mathrm{~s}^{-1}$ or even better. This setup will provide long term tracking of the FP and could also detect additional effects, e.g. aging of the Zerodur spacers or aging of the mirror coating. Both effects would change the effective distance of the mirrors and therefore change the spectrum over long timescales (years).

The external tracking project is already under development in our group within the ERC project Development of Radial Velocity Standards for the Search for Extrasolar Planets. 


\section{Bibliography}

Appenzeller, I., Fricke, K., Fürtig, W., Gässler, W., Häfner, R., Harke, R., Hess, H.-J., Hummel, W., Jürgens, P., Kudritzki, R.-P., Mantel, K.-H., Meisl, W., Muschielok, B., Nicklas, H., Rupprecht, G., Seifert, W., Stahl, O., Szeifert, T., and Tarantik, K. (1998). Successful commissioning of FORS1 - the first optical instrument on the VLT. The Messenger, 94:1-6.

Artigau, É., Donati, J.-F., and Delfosse, X. (2011). Planet Detection, Magnetic Field of Protostars and Brown Dwarfs Meteorology with SPIRou. In Johns-Krull, C., Browning, M. K., and West, A. A., editors, 16th Cambridge Workshop on Cool Stars, Stellar Systems, and the Sun, volume 448 of Astronomical Society of the Pacific Conference Series, page 771.

Avila, G. and Singh, P. (2008). Optical fiber scrambling and light pipes for high accuracy radial velocities measurements. In Society of Photo-Optical Instrumentation Engineers (SPIE) Conference Series, volume 7018 of Society of Photo-Optical Instrumentation Engineers (SPIE) Conference Series.

Avila, G., Singh, P., and Albertsen, M. (2006). Photometrical scrambling gain and focal ratio degradation in fibers for astronomical instruments. In Society of Photo-Optical Instrumentation Engineers (SPIE) Conference Series, volume 6269 of Society of Photo-Optical Instrumentation Engineers (SPIE) Conference Series.

Baranne, A., Queloz, D., Mayor, M., Adrianzyk, G., Knispel, G., Kohler, D., Lacroix, D., Meunier, J.-P., Rimbaud, G., and Vin, A. (1996). ELODIE: A spectrograph for accurate radial velocity measurements. A\&AS, 119:373-390.

Bean, J. L., Seifahrt, A., Hartman, H., Nilsson, H., Wiedemann, G., Reiners, A., Dreizler, S., and Henry, T. J. (2010). The CRIRES Search for Planets Around the Lowest-mass Stars. I. High-precision Near-infrared Radial Velocities with an Ammonia Gas Cell. ApJ, 713:410-422.

Butler, R. P., Marcy, G. W., Williams, E., McCarthy, C., Dosanjh, P., and Vogt, S. S. (1996). Attaining Doppler Precision of 3 M s-1. PASP, 108:500.

Chazelas, B., Pepe, F., Wildi, F., Bouchy, F., Perruchot, S., and Avila, G. (2010). New scramblers for precision radial velocity: square and octagonal fibers. In Society of Photo-Optical Instrumentation Engineers (SPIE) Conference Series, volume 7739 of Society of Photo-Optical Instrumentation Engineers (SPIE) Conference Series.

Clough, S. A., Iacono, M. J., and Moncet, J.-L. (1992). Line-by-Line Calculations of Atmospheric Fluxes and Cooling Rates: Application to Water Vapor. J. Geophys. Res., 97:15761. 
Drever, R. W. P., Hall, J. L., Kowalski, F. V., Hough, J., Ford, G. M., Munley, A. J., and Ward, H. (1983). Laser phase and frequency stabilization using an optical resonator. Applied Physics B: Lasers and Optics, 31(2):97-105.

Dumusque, X., Pepe, F., Lovis, C., Ségransan, D., Sahlmann, J., Benz, W., Bouchy, F., Mayor, M., Queloz, D., Santos, N., and Udry, S. (2012). An Earth-mass planet orbiting $\alpha$ Centauri B. Nature, 491:207-211.

Dürbye, J. (2013). Aufbau, Leistungsfähigkeit und Betrieb des Littrow-Spektrographen am Vakuum-Vertikal Teleskop. Diplomarbeit, Georg-August Universität Göttingen.

Hall, J. L. and Hänsch, T. W. (2005). History of optical comb development. In Jun, Y. and Cundiff, S. T., editors, Femtosecond Optical Frequency Comb Technology: Principle, Operation and Application. Springer Science Business Media.

Hauschildt, P. H., Baron, E., and Allard, F. (1997). Parallel Implementation of the PHOENIX Generalized Stellar Atmosphere Program. ApJ, 483:390.

Husser, T.-O. and Ulbrich, K. (2013). Using a model for telluric absorption in full-spectrum fits. ArXiv e-prints.

Klose, J. Z. and Bridges, J. M. (1987). Radiance of a Pt/Cr-Ne hollow cathode spectral line source. Appl. Opt., 26:5202-5203.

Kupka, F. G., Ryabchikova, T. A., Piskunov, N. E., Stempels, H. C., and Weiss, W. W. (2000). VALD-2 - The New Vienna Atomic Line Database. Baltic Astronomy, 9:590-594.

Lovis, C. and Pepe, F. (2007). A new list of thorium and argon spectral lines in the visible. A\&A, 468:1115-1121.

Mahadevan, S., Ramsey, L., Bender, C., Terrien, R., Wright, J. T., Halverson, S., Hearty, F., Nelson, M., Burton, A., Redman, S., Osterman, S., Diddams, S., Kasting, J., Endl, M., and Deshpande, R. (2012). The habitable-zone planet finder: a stabilized fiber-fed NIR spectrograph for the Hobby-Eberly Telescope. In Society of Photo-Optical Instrumentation Engineers (SPIE) Conference Series, volume 8446 of Society of Photo-Optical Instrumentation Engineers (SPIE) Conference Series.

Mayor, M., Bonfils, X., Forveille, T., Delfosse, X., Udry, S., Bertaux, J.-L., Beust, H., Bouchy, F., Lovis, C., Pepe, F., Perrier, C., Queloz, D., and Santos, N. C. (2009). The HARPS search for southern extra-solar planets. XVIII. An Earth-mass planet in the GJ 581 planetary system. A\&A, 507:487-494.

Mayor, M., Pepe, F., Queloz, D., Bouchy, F., Rupprecht, G., Lo Curto, G., Avila, G., Benz, W., Bertaux, J.-L., Bonfils, X., Dall, T., Dekker, H., Delabre, B., Eckert, W., Fleury, M., Gilliotte, A., Gojak, D., Guzman, J. C., Kohler, D., Lizon, J.-L., Longinotti, A., Lovis, C., Megevand, D., Pasquini, L., Reyes, J., Sivan, J.-P., Sosnowska, D., Soto, R., Udry, S., van Kesteren, A., Weber, L., and Weilenmann, U. (2003). Setting New Standards with HARPS. The Messenger, 114:20-24.

Mayor, M. and Queloz, D. (1995). A Jupiter-mass companion to a solar-type star. Nature, 378:355-359. 
Mégevand, D., Zerbi, F. M., Cabral, A., Di Marcantonio, P., Amate, M., Pepe, F., Cristiani, S., Rebolo, R., Santos, N. C., Dekker, H., Abreu, M., Affolter, M., Avila, G., Baldini, V., Bristow, P., Broeg, C., Carvas, P., Cirami, R., Coelho, J., Comari, M., Conconi, P., Coretti, I., Cupani, G., D'Odorico, V., De Caprio, V., Delabre, B., Figueira, P., Fleury, M., Fragoso, A., Genolet, L., Gomes, R., Gonzalez Hernandez, J., Hughes, I., Iwert, O., Kerber, F., Landoni, M., Lima, J., Lizon, J.-L., Lovis, C., Maire, C., Mannetta, M., Martins, C., Moitinho, A., Molaro, P., Monteiro, M., Rasilla, J. L., Riva, M., Santana Tschudi, S., Santin, P., Sosnowska, D., Sousa, S., Spanò, P., Tenegi, F., Toso, G., Vanzella, E., Viel, M., and Zapatero Osorio, M. R. (2012). ESPRESSO: the ultimate rocky exoplanets hunter for the VLT. In Society of Photo-Optical Instrumentation Engineers (SPIE) Conference Series, volume 8446 of Society of Photo-Optical Instrumentation Engineers (SPIE) Conference Series.

Owens, J. C. (1967). Optical refractive index of air: dependence on pressure, temperature, and composition. Appl. Opt., 6:51.

Paschen, F. (1916). Bohrs Heliumlinien. Annalen der Physik, 355:901-940.

Quirrenbach, A., Amado, P. J., Mandel, H., Caballero, J. A., Ribas, I., Reiners, A., Mundt, R., and CARMENES Consortium (2010). CARMENES: Calar Alto High-Resolution Search for M Dwarfs with Exo-earths with a Near-infrared Echelle Spectrograph. In Coudé du Foresto, V., Gelino, D. M., and Ribas, I., editors, Pathways Towards Habitable Planets, volume 430 of Astronomical Society of the Pacific Conference Series, page 521.

Redman, S., Nave, G., Lawler, J., Ramsey, L., and Mahadevan, S. (2010). Uranium-Neon as a Near-Infrared Calibration Source. In Astronomy of Exoplanets with Precise Radial Velocities, page $17 \mathrm{P}$.

Redman, S. L., Lawler, J. E., Nave, G., Ramsey, L. W., and Mahadevan, S. (2011). The Infrared Spectrum of Uranium Hollow Cathode Lamps from $850 \mathrm{~nm}$ to $4000 \mathrm{~nm}$ : Wavenumbers and Line Identifications from Fourier Transform Spectra. ApJS, 195:24.

Sato, B., Omiya, M., Liu, Y., Harakawa, H., Izumiura, H., Kambe, E., Toyota, E., Murata, D., Lee, B.-C., Masuda, S., Takeda, Y., Yoshida, M., Itoh, Y., Ando, H., Kokubo, E., Ida, S., Zhao, G., and Han, I. (2010). Substellar Companions to Evolved Intermediate-Mass Stars: HD 145457 and HD 180314. PASJ, 62:1063-.

Schäfer, S., Lenz, L. F., and Reiners, A. (2013). The influence of fibers and their illumination on the radial velocity stability of Fabry-Perot etalons. Submitted for publication in A\&A.

Schäfer, S. and Reiners, A. (2012). Two Fabry-Perot interferometers for high precision wavelength calibration in the near-infrared. In Society of Photo-Optical Instrumentation Engineers (SPIE) Conference Series, volume 8446 of Society of Photo-Optical Instrumentation Engineers (SPIE) Conference Series.

Seifahrt, A., Käufl, H. U., Zängl, G., Bean, J. L., Richter, M. J., and Siebenmorgen, R. (2010). Synthesising, using, and correcting for telluric features in high-resolution astronomical spectra . A near-infrared case study using CRIRES. A\&A, 524:A11.

Strassmeier, K. G., Woche, M., Ilyin, I., Popow, E., Bauer, S.-M., Dionies, F., Fechner, T., Weber, M., Hofmann, A., Storm, J., Materne, R., Bittner, W., Bartus, J., Granzer, T., Denker, C., Carroll, T., Kopf, M., DiVarano, I., Beckert, E., and Lesser, M. (2008). PEPSI: the Potsdam 
Echelle Polarimetric and Spectroscopic Instrument for the LBT. In Society of Photo-Optical Instrumentation Engineers (SPIE) Conference Series, volume 7014 of Society of Photo-Optical Instrumentation Engineers (SPIE) Conference Series.

ten Bruggencate, P. and Jäger, F. W. (1951). Das Turmteleskop der Göttinger Sternwarte. Veroeffentlichungen der Universitaets-Sternwarte zu Göettingen, 6:1-28.

Vernet, J., Dekker, H., D’Odorico, S., Kaper, L., Kjaergaard, P., Hammer, F., Randich, S., Zerbi, F., Groot, P. J., Hjorth, J., Guinouard, I., Navarro, R., Adolfse, T., Albers, P. W., Amans, J.-P., Andersen, J. J., Andersen, M. I., Binetruy, P., Bristow, P., Castillo, R., Chemla, F., Christensen, L., Conconi, P., Conzelmann, R., Dam, J., de Caprio, V., de Ugarte Postigo, A., Delabre, B., di Marcantonio, P., Downing, M., Elswijk, E., Finger, G., Fischer, G., Flores, H., François, P., Goldoni, P., Guglielmi, L., Haigron, R., Hanenburg, H., Hendriks, I., Horrobin, M., Horville, D., Jessen, N. C., Kerber, F., Kern, L., Kiekebusch, M., Kleszcz, P., Klougart, J., Kragt, J., Larsen, H. H., Lizon, J.-L., Lucuix, C., Mainieri, V., Manuputy, R., Martayan, C., Mason, E., Mazzoleni, R., Michaelsen, N., Modigliani, A., Moehler, S., Møller, P., Norup Sørensen, A., Nørregaard, P., Péroux, C., Patat, F., Pena, E., Pragt, J., Reinero, C., Rigal, F., Riva, M., Roelfsema, R., Royer, F., Sacco, G., Santin, P., Schoenmaker, T., Spano, P., Sweers, E., Ter Horst, R., Tintori, M., Tromp, N., van Dael, P., van der Vliet, H., Venema, L., Vidali, M., Vinther, J., Vola, P., Winters, R., Wistisen, D., Wulterkens, G., and Zacchei, A. (2011). $\mathrm{X}$-shooter, the new wide band intermediate resolution spectrograph at the ESO Very Large Telescope. A\&A, 536:A105.

Walsh, A. (1956). The application of atomic absorption spectra to chemical analysis. Spectrochimica Acta, 7:108-117.

Wildi, F., Pepe, F., Chazelas, B., Lo Curto, G., and Lovis, C. (2010). A Fabry-Perot calibrator of the HARPS radial velocity spectrograph: performance report. In Society of Photo-Optical Instrumentation Engineers (SPIE) Conference Series, volume 7735 of Society of Photo-Optical Instrumentation Engineers (SPIE) Conference Series.

Wilken, T. (2010). Calibrating Astronomical Spectrographs with Frequency Combs. PhD thesis.

Wilken, T., Lo Curto, G., Probst, R. A., Steinmetz, T., Manescau, A., Pasquini, L., González Hernández, J. I., Rebolo, R., Hänsch, T. W., Udem, T., and Holzwarth, R. (2012). A spectrograph for exoplanet observations calibrated at the centimetre-per-second level. Nature, 485:611614 . 


\section{thank you notes}

Danke Ansgar, für deine Betreuung und dein Vertrauen mir nahezu unbegrenzte Freiheiten einzuräumen. Danke außerde für deine immer offen stehende Tür und die Möglichkeit, mit jeder Frage zu dir zu kommen.

Danke Stefan und Uwe, für die vielen hilfreichen Diskussionen und eure Unterstützung im Rahmen des GRK.

Danke an Christof Schmidt für die Hilfe beim Design des Vakuumtanks und dessen Konstruktion.

Danke an Peter Jeep und die Mitarbeiter der Werkstatt des Instituts für Astrophysik für zahllose Sonderanfertigungen und Unterstützung bei technischen Fragen.

Danke an Julian Stürmer für das Präparieren und Einkleben der Fasern in den Vakuumfeedthrough sowie für das Beantworten zahlreicher Fragen zum Thema Faseroptik.

Danke an die Mitarbeiter der Landessternwarte Tautenburg, insbesondere Eike Günther und Johannes Winkler, für die Hilfe bei den Testaufnahmen in Tautenburg.

Danke an Ulrike Lemke und Julian Dürbye für Unterstützung im Optiklabor.

Danke an Katrin Ulbrich und Tim-Oliver Husser für die Atmosphärenmodelle und deren Anpassung an die PHOENIX und XSHOOTER Spektren.

Danke an Matthias Zecheister für die XSHOOTER Datenreduktion und diverse Diskussionen zum Thema Fabry-Pérot.

Danke an Ulf für das Korrekturlesen dieser Arbeit.

Danke Feo, für Diskussionen und Entwicklung von Ideen und Versuchsaufbauten bereits am Frühstückstisch. 